\title{
Model-independent bounds on new physics effects in non-leptonic tree-level decays of B-mesons
}

\author{
Alexander Lenz $^{a}$ and Gilberto Tetlalmatzi-Xolocotzi ${ }^{b, c, 1}$ \\ ${ }^{a}$ Institute for Particle Physics Phenomenology, Durham University, \\ DH1 3LE Durham, United Kingdom \\ ${ }^{b}$ Theoretische Physik 1, Naturwissenschaftlich-Technische Fakultät, Universität Siegen, \\ Walter-Flex-Strasse 3, D-57068 Siegen, Germany \\ ${ }^{c}$ Nikhef, Theory Group, \\ Science Park 105, 1098 XG Amsterdam, The Netherlands \\ E-mail: alexander.lenz@durham.ac.uk, gtx@physik.uni-siegen.de
}

ABSTRACT: We present a considerably improved analysis of model-independent bounds on new physics effects in non-leptonic tree-level decays of B-mesons. Our main finding is that contributions of about \pm 0.1 to the Wilson coefficient of the colour-singlet operator $Q_{2}$ of the effective weak Hamiltonian and contributions in the range of \pm 0.5 (both for real and imaginary part) to $Q_{1}$ can currently not be excluded at the $90 \%$ C.L. Effects of such a size can modify the direct experimental extraction of the CKM angle $\gamma$ by up to $10^{\circ}$ and they could lead to an enhancement of the decay rate difference $\Delta \Gamma_{d}$ of up to a factor of 5 over its SM value - a size that could explain the D0 dimuon asymmetry. Future more precise measurements of the semi-leptonic asymmetries $a_{s l}^{q}$ and the lifetime ratio $\tau\left(B_{s}\right) / \tau\left(B_{d}\right)$ will allow to shrink the bounds on tree-level new physics effects considerably. Due to significant improvements in the precision of the non-perturbative input we update all SM predictions for the mixing obervables in the course of this analysis, obtaining: $\Delta M_{s}=(18.77 \pm 0.86) \mathrm{ps}^{-1}, \Delta M_{d}=(0.543 \pm 0.029) \mathrm{ps}^{-1}, \Delta \Gamma_{s}=(9.1 \pm 1.3) \cdot 10^{-2} \mathrm{ps}^{-1}$, $\Delta \Gamma_{d}=(2.6 \pm 0.4) \cdot 10^{-3} \mathrm{ps}^{-1}, a_{s l}^{s}=(2.06 \pm 0.18) \cdot 10^{-5}$ and $a_{s l}^{d}=(-4.73 \pm 0.42) \cdot 10^{-4}$.

KeYwords: Beyond Standard Model, CP violation, Heavy Quark Physics

ArXiv EPrint: 1912.07621

\footnotetext{
${ }^{1}$ Corresponding author.
} 


\section{Contents}

1 Introduction 1

2 Basic formalism 3

2.1 Effective Hamiltonian 3

2.2 Heavy Quark Expansion 5

$\begin{array}{lll}2.3 \text { QCD factorization } & 7\end{array}$

$\begin{array}{llr}3 & \text { Strategy } & 11\end{array}$

$\begin{array}{lll}3.1 & \text { Statistical analysis } & 12\end{array}$

4 Individual constraints $\quad 13$

4.1 Observables constraining $b \rightarrow c \bar{u} d$ transitions $\quad 14$

4.1.1 $\quad \bar{B}_{d}^{0} \rightarrow D^{*+} \pi^{-} \quad 14$

4.2 Observables constraining $b \rightarrow u \bar{u} d$ transitions $\quad 16$

$\begin{array}{lll}4.2 .1 & R_{\pi \pi} & 16\end{array}$

$\begin{array}{lll}4.2 .2 & S_{\pi \pi} & 19\end{array}$

$\begin{array}{lll}4.2 .3 & S_{\rho \pi} & 21\end{array}$

$\begin{array}{lll}4.2 .4 & R_{\rho \rho} & 23\end{array}$

4.3 Observables constraining $b \rightarrow c \bar{c} s$ transitions 24

$\begin{array}{lll}\text { 4.3.1 } & \bar{B} \rightarrow X_{s} \gamma & 25\end{array}$

$\begin{array}{lll}\text { 4.3.2 } & \Delta \Gamma_{s} \text { : bounds and SM update } & 28\end{array}$

$\begin{array}{lll}4.3 .3 & S_{J / \psi \phi} & 34\end{array}$

$\begin{array}{lll}4.3 .4 & \tau_{B_{s}} / \tau_{B_{d}} & 35\end{array}$

4.4 Observables constraining $b \rightarrow c \bar{c} d$ transitions 36

4.4.1 $\sin \left(2 \beta_{d}\right)$ and SM update of $\Delta M_{q} \quad 36$

$\begin{array}{lll}\text { 4.4.2 } \quad \bar{B} \rightarrow X_{d} \gamma & 40\end{array}$

$\begin{array}{lll}4.5 & \text { Observables constraining multiple channels } & 41\end{array}$

4.5.1 $a_{s l}^{s}$ and $a_{s l}^{d}$ : bounds and SM update 42

5 Global $\chi^{2}$-fit results $\quad 43$

$5.1 \chi^{2}$-fit for the $b \rightarrow u \bar{u} d$ channel and bounds on $\Delta \Gamma_{d}$

$5.2 \chi^{2}$-fit for the $b \rightarrow c \bar{u} d$ channel and bounds on $\Delta \Gamma_{d} \quad 47$

$5.3 \chi^{2}$-fit for the $b \rightarrow c \bar{c} d$ channel and bounds on $\Delta \Gamma_{d} \quad 47$

5.4 Universal fit on $\Delta C_{1}\left(M_{W}\right)$ and $\Delta C_{2}\left(M_{W}\right) \quad 48$

5.5 NP in non-leptonic tree-level decays and its interplay with the CKM angle $\gamma \quad 50$

$6 \quad$ Future prospects $\quad \mathbf{5 2}$

$6.1 \tau_{B_{s}} / \tau_{B_{d}} \quad 52$

6.2 Semi-leptonic CP asymmetries 53

6.3 Rare decays 53 
$\begin{array}{ll}\text { A Numerical inputs } & 56\end{array}$

B QCD-factorization formulas $\quad 60$

$\begin{array}{lll}\text { B.1 Generic parameters } & 60\end{array}$

B.1.1 Vertices for the $B \rightarrow \pi \pi, \rho \pi, \pi \rho, \rho \rho$ decays $\quad 60$

B.1.2 Vertices for the $B \rightarrow J / \psi \phi$ decay 61

B.1.3 Penguin functions 61

B.1.4 Hard scattering functions for the $B \rightarrow \pi \pi, \rho \pi, \pi \rho, \rho \rho$ decays 64

B.1.5 Hard scattering function for the $B \rightarrow J / \psi \phi \quad 64$

B.2 Annihilation coefficients 66

B.3 Annihilation kernels 66

\section{Introduction}

Motivations for flavour physics are manifold. Standard model parameters, like the elements of the Cabibbo-Kobayashi-Maskawa (CKM) matrix [1,2] or quark masses are determined very accurately in this field. Moreover the quark-sector is the only sector, where CP violating effects have been detected so far — since 1964 in the Kaon sector [3] and since 2001 also in the B-sector $[4,5]$. Very recently $\mathrm{CP}$ violation has been measured for the first time in the charm sector [6], which might actually be an indication for physics beyond the standard model (BSM) $[7,8]$. Considering that $\mathrm{CP}$ violation is a necessary ingredient for creating a baryon asymmetry in the universe [9], flavour physics might shed some light on this unsolved problem. In addition flavour physics is perfectly suited for indirect new physics (NP) searches, because there are many processes strongly suppressed in the standard model (SM) but not necessarily in hypothetical NP models. And, last but not least, a comparison between experiment and theory predictions can provide a deeper insight into the dynamics of QCD.

In recent years experimental flavour physics entered a new precision era, which was initiated by the B-factories at KEK and SLAC (see e.g. [10]) and the Tevatron at Fermilab [11, 12]. Currently this field is dominated by the results of the LHCb collaboration $[13,14]$, but also complemented by competing results from the general purpose detectors ATLAS and CMS, see e.g. [15, 16].

The corresponding dramatic increase in experimental precision, demands complementary improvements in theory. Besides calculating higher orders in perturbative QCD or more precise lattice evaluations, this also means revisiting some common approximations by investigating questions like: how large are penguin contributions? How well does QCDfactorization [17-20] work? How large can duality violation in the Heavy Quark Expansion (HQE) (see e.g. [21-28] for pioneering papers and [29] for a recent review) be? How sizeable NP effects in tree-level decays can be? Some of these questions have been studied in detail for quite some time. There is e.g. a huge literature on penguin contributions, see e.g. [30, 31] for reviews. Others gained interest recently, for instance duality violations [32]. 
In principle all these questions are interwoven, but as a starting point it is reasonable to consider them separately. The assumption of no NP effects at tree-level in non-leptonic $b$-decays was already challenged after the measurement of the dimuon asymmetry by the D0-collaboration [33-36], see e.g. [37]. And after the measurements of $B \rightarrow D^{(*)} \tau \nu$ by BaBar, Belle and LHCb [38-41] for the case of semi-leptonic $b$-decays.

Compared to numerous systematic studies of NP effects in the Wilson coefficients of the electromagnetic dipole and the semi-leptonic penguin operators $Q_{7 \gamma}$ and $Q_{9}, Q_{10}$ respectively, see e.g. [42-54], we are not aware of systematic studies for NP effects in the Wilson coefficients for non-leptonic tree-level decays, except the ones in [55-60]. ${ }^{1}$

The aim of the current paper is to considerably extend the studies in [55, 57] by incorporating two main improvements:

1. A full $\chi^{2}$-fit is performed instead of a simple parameter scan. To implement this step we use the package MyFitter [63] and allow the different nuisance parameters to run independently. This will allow us to account properly for the corresponding statistical correlations.

2. Instead of simplified theoretical equations we include full expressions for the observables under investigation.

The recent work in $[58,60]$ concentrates exclusively on the transition $b \rightarrow c \bar{c} s$, while we consider in this paper all different hadronic decays, that occur in the SM on tree-level. Moreover in this work we consider only BSM effects to the tree-level operators $Q_{1}$ and $Q_{2}$, while $[58,60]$ investigates also effects of four-quark operators that do not exist in the SM. Whenever there is some direct overlap between the work in $[58,60]$ we directly compare the results. Any realistic BSM model that gives rise to new tree-level effects will also give new effects at the loop-level, which are not considered in the current model independent approach. In that respect this work can be considered as an important building block of future model dependent studies.

The paper is organised as follows: in section 2 we describe briefly the theoretical tools to be used: we start with the effective Hamiltonian in section 2.1, then in section 2.2 we introduce the Heavy Quark Expansion and in section 2.3 we review basic concepts in QCD factorization relevant to this project. Next in section 3, we outline our strategy for performing the $\chi^{2}$-fit. We discuss all our different constraints on NP effects in non-leptonic tree-level decays in section 4 . The bounds on individual decay channels are organized as follows: $b \rightarrow c \bar{u} d$ in section $4.1, b \rightarrow u \bar{u} d$ in section $4.2, b \rightarrow c \bar{c} s$ in section $4.3, b \rightarrow c \bar{c} d$ in section 4.4. Additionally, in section 4.5 we present observables constraining more decay channels. Our main results are presented in section 5: fits for the allowed size of BSM effects in the tree-level Wilson coefficients based on individual decay channels will be discussed in sections 5.1-5.3. In particular we focus on the channels which can enhance the decay rate difference of neutral $B_{d}^{0}$-mesons $\Delta \Gamma_{d}$ and we calculate these enhancements. Flavouruniversal bounds on the tree level Wilson coefficients will be presented in section 5.5, with

\footnotetext{
${ }^{1}$ In [61] NP entering inside $Q_{5}$ and $Q_{6}$ is explored, establishing a link between the $B \rightarrow K \pi$ puzzle and the $\varepsilon^{\prime} / \varepsilon$ ratio. This is further addressed in [62] within the context of simplified $Z^{\prime}$ models with $\mathrm{U}(2)^{3}$ flavour symmetry.
} 
an emphasis on the consequences of tree-level NP effects on the precision in the direct extraction of the CKM angle $\gamma$. In section 6 we study observables that seem to be most promising in shrinking the space for new effects in $C_{1}$ and $C_{2}$. Finally we conclude in section 7 and give additional information in the appendices.

Since there has been tremendous progress (see e.g. [64, 65]) in the theoretical precision of the mixing observables, we will present in this work numerical updates of all mixing observables: $\Delta \Gamma_{q}$ in section 4.3.2, $\Delta M_{q}$ in section 4.4.1 and the semi-leptonic CP asymmetries $a_{s l}^{q}$ and mixing phases $\phi_{q}$ in section 4.5.

\section{Basic formalism}

In this section we provide an overview of the basic theoretical tools required for the description of our different flavour observables, this includes: the effective Hamiltonian, the Heavy Quark Expansion for inclusive decays and mixing observables. A quick review of QCD factorization for exclusive, non-leptonic decays is also provided. In addition we fix the notation to be used during this work.

\subsection{Effective Hamiltonian}

We start by introducing the effective Hamiltonian describing a $b$-quark decay into a $p \bar{p}^{\prime} q$ final state via electroweak interactions, with $p, p^{\prime}=u, c$ and $q=s, d$ :

$$
\begin{aligned}
\hat{\mathcal{H}}_{\mathrm{eff}}^{|\Delta B|=1}= & \frac{G_{F}}{\sqrt{2}}\left\{\sum_{p, p^{\prime}=u, c} \lambda_{p p^{\prime}}^{(q)} \sum_{i=1,2} C_{i}^{q, p p^{\prime}}(\mu) \hat{Q}_{i}^{q, p p^{\prime}}\right. \\
& \left.+\sum_{p=u, c} \lambda_{p}^{(q)}\left[\sum_{i=3}^{10} C_{i}^{q}(\mu) \hat{Q}_{i}^{q}+C_{7 \gamma}^{q} \hat{Q}_{7 \gamma}^{q}+C_{8 g}^{q} \hat{Q}_{8 g}^{q}\right]\right\}+ \text { h.c. }
\end{aligned}
$$

The Fermi constant is denoted by $G_{F}$, additionally we have introduced the following CKM combinations

$$
\begin{aligned}
& \lambda_{p}^{(q)}=V_{p b} V_{p q}^{*}, \\
& \lambda_{p p^{\prime}}^{(q)}=V_{p b} V_{p^{\prime} q}^{*} .
\end{aligned}
$$

Moreover $C_{i}$ denote the Wilson coefficients of the following dimension six operators:

$$
\begin{aligned}
\hat{Q}_{1}^{q, p p^{\prime}} & =\left(\overline{\hat{p}}_{\beta} \hat{b}_{\alpha}\right)_{V-A}\left(\overline{\hat{q}}_{\alpha} \hat{p}_{\beta}^{\prime}\right)_{V-A}, & \hat{Q}_{2}^{q, p p^{\prime}} & =(\overline{\hat{p}} \hat{b})_{V-A}\left(\overline{\hat{q}} \hat{p}^{\prime}\right)_{V-A}, \\
\hat{Q}_{3}^{q} & =(\overline{\hat{q}} \hat{b})_{V-A} \sum_{k}(\overline{\hat{k}} \hat{k})_{V-A}, & \hat{Q}_{4}^{q} & =\left(\overline{\hat{q}}_{\alpha} \hat{b}_{\beta}\right)_{V-A} \sum_{k}\left(\overline{\hat{k}}_{\beta} \hat{k}_{\alpha}\right)_{V-A}, \\
\hat{Q}_{5}^{q} & =(\overline{\hat{q}} \hat{b})_{V-A} \sum_{k}(\overline{\hat{k}} \hat{k})_{V+A}, & \hat{Q}_{6}^{q} & =\left(\overline{\hat{q}}_{\alpha} \hat{b}_{\beta}\right)_{V-A} \sum_{k}\left(\overline{\hat{k}}_{\beta} \hat{k}_{\alpha}\right)_{V+A}, \\
\hat{Q}_{7}^{q} & =(\overline{\hat{q}} \hat{b})_{V-A} \sum_{k} \frac{3}{2} e_{k}(\overline{\hat{k}} \hat{k})_{V+A}, & \hat{Q}_{8}^{q} & =\left(\overline{\hat{q}}_{\alpha} \hat{b}_{\beta}\right)_{V-A} \sum_{k} \frac{3}{2} e_{k}\left(\overline{\hat{k}}_{\beta} \hat{k}_{\alpha}\right)_{V+A}, \\
\hat{Q}_{9}^{q} & =(\overline{\hat{q} \hat{b}})_{V-A} \sum_{k} \frac{3}{2} e_{k}(\overline{\hat{k}} \hat{k})_{V-A}, & \hat{Q}_{10}^{q} & =\left(\overline{\hat{q}}_{\alpha} \hat{b}_{\beta}\right)_{V-A} \sum_{k} \frac{3}{2} e_{k}\left(\overline{\hat{k}}_{\beta} \hat{k}_{\alpha}\right)_{V-A}, \\
\hat{Q}_{7 \gamma}^{q} & =\frac{e}{8 \pi^{2}} m_{b} \overline{\hat{q}} \sigma_{\mu \nu}(1+\gamma)_{5} \hat{F}^{\mu \nu} \hat{b}, & \hat{Q}_{8 g}^{q} & =\frac{g_{s}}{8 \pi^{2}} m_{b} \overline{\hat{q}} \sigma_{\mu \nu}\left(1+\gamma_{5}\right) \hat{G}^{\mu \nu} \hat{b} .
\end{aligned}
$$


Here $\alpha$ and $\beta$ are colour indices, $e_{k}$ is the electric charge of the quark $k$ (in the penguin operators the quark flavours are summed over $k=u, d, s, c, b), e$ is the $\mathrm{U}(1)_{\mathrm{em}}$ coupling and $g_{s}$ the $\mathrm{SU}(3)_{C}$ one, $m_{b}$ is the mass of the $b$-quark and $F^{\mu \nu}$ and $G^{\mu \nu}$ are the electro-magnetic and chromo-magnetic field strength tensors respectively. In this work we consider NP effects that will affect the tree-level operators $\hat{Q}_{1}^{q, p p^{\prime}}$ and $\hat{Q}_{2}^{q, p p^{\prime}}$ by modifying their corresponding Wilson coefficients. In our notation $\hat{Q}_{1}^{q, p p^{\prime}}$ is colour non diagonal and $\hat{Q}_{2}^{q, p p^{\prime}}$ is the colour singlet, the QCD penguin operators correspond to $\hat{Q}_{3-6}^{q}$ and the electro-weak penguin interactions are described by $\hat{Q}_{7-10}^{q}$. Different bases compared to the one in eq. (2.3) are used in the literature. Our notation agrees with the one used in [66] and [67], here $C_{8 g}$ is negative because we are considering $-i g \gamma_{\mu} T^{a}$ as the Feynman rule for the quark-gluon vertex. In [19] a different basis is used, where $\hat{Q}_{1}$ and $\hat{Q}_{2}$ are interchanged and $\hat{Q}_{7 \gamma}$ and $\hat{Q}_{8 g}$ have a different sign (this is equivalent to the sign convention $i D^{\mu}=i \partial^{\mu}+g_{s} A_{a}^{\mu} T^{a}$ for the gauge-covariant derivative). ${ }^{2}$ A nice introduction on effective Hamiltonians can be found in [69], and a concise review up to NLO-QCD in [66].

The Wilson coefficients $C_{i}$ with $i=1,2, \ldots, 10,7 \gamma, 8 g$ in eq. (2.1) are obtained by matching the calculations of the effective theory and the full SM at the scale $\mu=M_{W}$ and then evolving down to the scale $\mu \sim m_{b}$ using the renormalisation group equations according to

$$
\vec{C}(\mu)=\boldsymbol{U}\left(\mu, M_{W}, \alpha\right) \vec{C}\left(M_{W}\right),
$$

where the NLO evolution matrix is given by [19]

$$
\boldsymbol{U}\left(\mu, M_{W}, \alpha\right)=\boldsymbol{U}\left(\mu, \mu_{W}\right)+\frac{\alpha}{4 \pi} \boldsymbol{R}\left(\mu, \mu_{W}\right) .
$$

The matrix $\mathbf{U}\left(\mu, \mu_{W}\right)$ accounts for pure QCD evolution, on the other hand $\mathbf{R}\left(\mu, \mu_{W}\right)$ introduces QED effects as well. We write at NLO [19]

$$
\begin{aligned}
\boldsymbol{U}\left(\mu, M_{W}, \alpha\right)= & {\left[\boldsymbol{U}_{0}+\frac{\alpha_{s}(\mu)}{4 \pi} \boldsymbol{J} \boldsymbol{U}_{0}-\frac{\alpha_{s}\left(M_{W}\right)}{4 \pi} \boldsymbol{U}_{0} \boldsymbol{J}\right.} \\
& \left.+\frac{\alpha}{4 \pi}\left(\frac{4 \pi}{\alpha_{s}(\mu)} \boldsymbol{R}_{0}+\boldsymbol{R}_{1}\right)\right]
\end{aligned}
$$

where $\alpha_{s}(\mu)$ denotes the strong coupling at the scale $\mu$ calculated up to NLO-QCD precision and $\alpha$ is the electro-magnetic coupling. The matrix $\boldsymbol{U}_{0}$ is the LO of the pure QCD evolution component $\mathbf{U}\left(\mu, \mu_{W}\right)$. At $\mathrm{LO}$ the evolution matrix $\boldsymbol{U}\left(\mu, M_{W}, \alpha\right)$ reduces to

$$
\boldsymbol{U}^{\mathrm{LO}}\left(\mu, \mu_{W}, \alpha\right)=\boldsymbol{U}_{0}+\frac{\alpha}{\alpha_{s}(\mu)} \boldsymbol{R}_{0} .
$$

The NLO-QCD corrections are then introduced through $\boldsymbol{J}$. The explicit expressions for $\boldsymbol{U}_{0}$ and $\boldsymbol{J}$ are given in eqs. (3.94)-(3.98) of [66]. The anomalous dimension matrices $\gamma_{s}^{(0)}$ and $\gamma_{s}^{(1)}$ required for these evaluations can be found in eq. (6.25) and tables XIV and

\footnotetext{
${ }^{2} \mathrm{~A}$ minimal basis of dimension six operators for $\Delta B \neq 0$ processes has been introduced in [68]. This extends our set of operators in eq. (2.3). For the purposes of studying NP in tree-level non-leptonic operators the basis in eq. (2.3) is enough. However, future extension which include NP in other operators as well, should be done paying attention to the results presented in [68].
} 
XV of [66]. To introduce QED corrections we calculate $\mathbf{R}_{\mathbf{0}}$ and $\mathbf{R}_{\mathbf{1}}$ using eqs. (7.24)-(7.28) of [66], the anomalous dimension matrices used are $\gamma_{e}^{(0)}$ and $\gamma_{e}^{(1)}$ and are given in tables XVI and XVII of [66].

The initial conditions for the Wilson coefficients have the following expansion at NLO

$$
\begin{aligned}
\vec{C}\left(M_{W}\right)= & \vec{C}_{s}^{(0)}\left(M_{W}\right)+\frac{\alpha_{s}\left(M_{W}\right)}{4 \pi} \vec{C}_{s}^{(1)}\left(M_{W}\right) \\
& +\frac{\alpha}{4 \pi}\left[\vec{C}_{e}^{(0)}\left(M_{W}\right)+\frac{\alpha_{s}\left(M_{W}\right)}{4 \pi} \vec{C}_{e}^{(1)}\left(M_{W}\right)+\vec{R}_{e}^{(0)}\left(M_{W}\right)\right]
\end{aligned}
$$

as pointed out in [19] the electroweak contributions $\vec{C}_{e}^{(0)}$ and $\vec{C}_{e}^{(1)}$ in eq. (2.8) can be $x_{t}$ and/or $1 / \sin ^{2} \theta_{W}$ enhanced. Consequently it is fair to treat the product between $\alpha$ and $\vec{C}_{e}^{(0)}$ as a LO contribution and the product between $\alpha$ and $\vec{C}_{e}^{(1)}$ as a NLO effect. The remainder, denoted by $\vec{R}_{e}^{(0)}$, is numerically smaller in comparison with $\vec{C}_{e}^{(0)}$ and it is therefore treated as a NLO effect, it contains the NLO scheme dependency. This approach differs from the one followed by [66], where the contribution of $\vec{C}_{e}^{(0)}\left(M_{W}\right)+\vec{R}_{e}^{(0)}\left(M_{W}\right)$ is introduced as a NLO effect and then $\vec{C}_{e}^{(1)}$ is omitted. The explicit expressions for $\vec{C}_{s}^{(0)}, \vec{C}_{s}^{(1)}, \vec{C}_{e}^{(0)}, \vec{C}_{e}^{(1)}$ and $\vec{R}_{e}^{(0)}$ of $\vec{C}\left(M_{W}\right)$ are given in section VII.B of [66] and section 3.1 of [19], the results presented for $\vec{C}_{e}^{(1)}$ in [19] are based on the calculations of [70].

It should be further stressed that when applying eq. (2.4) we consistently dropped products between NLO contributions from $\boldsymbol{U}\left(\mu, M_{W}, \alpha\right)$ and NLO effects from $\vec{C}\left(M_{W}\right)$ but we have taken into account products between NLO contributions from $\boldsymbol{U}\left(\mu, M_{W}, \alpha\right)$ and LO contributions from $\vec{C}\left(M_{W}\right)$ and vice versa.

\subsection{Heavy Quark Expansion}

The effective Hamiltonian can be used to calculate inclusive decays of a heavy hadron $B_{q}$ into an inclusive final state $X$ via

$$
\Gamma\left(B_{q} \rightarrow X\right)=\frac{1}{2 m_{B_{q}}} \sum_{X} \int_{\mathrm{PS}}(2 \pi)^{4} \delta^{(4)}\left(p_{B_{q}}-p_{X}\right)\left|\left\langle X\left|\hat{\mathcal{H}}_{\mathrm{eff}}\right| B_{q}\right\rangle\right|^{2} .
$$

With the help of the optical theorem the total decay rate in eq. (2.9) can be rewritten as

$$
\Gamma\left(B_{q} \rightarrow X\right)=\frac{1}{2 m_{B_{q}}}\left\langle B_{q}|\hat{\mathcal{T}}| B_{q}\right\rangle
$$

with the transition operator

$$
\hat{\mathcal{T}}=\operatorname{Im} i \int d^{4} x \hat{T}\left[\hat{\mathcal{H}}_{\text {eff }}(x) \hat{\mathcal{H}}_{\text {eff }}(0)\right]
$$

consisting of a non-local double insertion of the effective Hamiltonian. Expanding this bi-local object in local operators gives the Heavy Quark Expansion (see e.g. [21-28] for pioneering papers and [29] for a recent review). The total decay rate $\Gamma$ of a $b$-hadron 
can then be expressed as products of perturbatively calculable coefficients $\Gamma_{i}$ times nonperturbative matrix elements $\left\langle O_{D}\right\rangle$ of $\Delta B=0$-operators of dimension $D=i+3$ :

$$
\begin{aligned}
\Gamma= & \Gamma_{0}\left\langle\hat{O}_{D=3}\right\rangle+\Gamma_{2} \frac{\left\langle\hat{O}_{D=5}\right\rangle}{m_{b}^{2}}+\tilde{\Gamma}_{3} \frac{\left\langle\tilde{\hat{O}}_{D=6}\right\rangle}{m_{b}^{3}}+\ldots \\
& +16 \pi^{2}\left[\Gamma_{3} \frac{\left\langle\hat{O}_{D=6}\right\rangle}{m_{b}^{3}}+\Gamma_{4} \frac{\left\langle\hat{O}_{D=7}\right\rangle}{m_{b}^{4}}+\Gamma_{5} \frac{\left\langle\hat{O}_{D=8}\right\rangle}{m_{b}^{5}}+\ldots\right]
\end{aligned}
$$

with $\left\langle\hat{O}_{D}\right\rangle=\left\langle B_{q}\left|\hat{O}_{D}\right| B_{q}\right\rangle /\left(2 M_{B_{q}}\right)$. The leading term $\Gamma_{0}$ describes the decay of a free $b$ quark and is free of non-perturbative uncertainties, since $\left\langle\hat{O}_{D=3}\right\rangle=1+\mathcal{O}\left(\left\langle\hat{O}_{D=5}\right\rangle / m_{b}^{2}\right)$. At order $1 / m_{b}^{2}$ small corrections due to the kinetic and chromomagnetic operator are arising, at order $1 / m_{b}^{3}$ we get e.g. the Darwin term in $\tilde{\Gamma}_{3}$, but also phase space enhanced terms $\Gamma_{3}$, stemming from weak exchange, weak annihilation and Pauli interference. The numerical values of the matrix elements are expected to be of the order the hadronic scale $\Lambda_{\mathrm{QCD}}$, thus the HQE is an expansion in the small parameter $\Lambda_{\mathrm{QCD}} / m_{b}$. Each of the terms $\Gamma_{i}$ with $i=0,2,3, \ldots$ can be expanded as

$$
\Gamma_{i}=\Gamma_{i}^{(0)}+\frac{\alpha_{s}}{4 \pi} \Gamma_{i}^{(1)}+\left(\frac{\alpha_{s}}{4 \pi}\right)^{2} \Gamma_{i}^{(2)}+\ldots
$$

In our investigation of the lifetimes we will use $\Gamma_{0}^{(0)}$ and $\Gamma_{0}^{(1)}$ from [71], which is based on [72-77], $\Gamma_{3}^{(0)}$ from [58] based on $[78,79]$ and $\Gamma_{3}^{(1)}$ from $[80,81]$. The matrix elements of the dimension six operators were recently determined in [82].

The HQE can also be used to describe the off-diagonal element $\Gamma_{12}$ of the meson mixing matrix

$$
\Gamma_{12}^{q}=\left[\Gamma_{12,3}^{q,(0)}+\frac{\alpha_{s}}{4 \pi} \Gamma_{12,3}^{q,(1)}+\ldots\right] \frac{\left\langle\hat{Q}_{D=6}\right\rangle}{m_{b}^{3}}+\left[\Gamma_{12,4}^{q,(0)}+\frac{\alpha_{s}}{4 \pi} \Gamma_{12,4}^{q,(1)}+\ldots\right] \frac{\left\langle\hat{Q}_{D=7}\right\rangle}{m_{b}^{4}}+\ldots
$$

with $\left\langle\hat{Q}_{D}\right\rangle=\left\langle B_{q}\left|\hat{Q}_{D}\right| \bar{B}_{q}\right\rangle /\left(2 M_{B_{q}}\right)$, where $\hat{Q}_{D}$ are $\Delta B=2$-operators of dimension $D$. The matrix element $\Gamma_{12}^{q}$ can be used together with $M_{12}^{q}$ to predict physical observables like mass differences, decay rate differences or semi-leptonic CP-asymmetries, see e.g. [31]

$$
\begin{aligned}
\Delta M_{q} & =2\left|M_{12}^{q}\right|, \\
\Delta \Gamma_{q} & =2\left|\Gamma_{12}^{q}\right| \cos \phi_{12}^{q}=-\operatorname{Re}\left(\frac{\Gamma_{12}^{q}}{M_{12}^{q}}\right) \Delta M_{q}, \\
a_{s l}^{q} & =\left|\frac{\Gamma_{12}^{q}}{M_{12}^{q}}\right| \sin \phi_{12}^{q}=\operatorname{Im}\left(\frac{\Gamma_{12}^{q}}{M_{12}^{q}}\right),
\end{aligned}
$$

with the phase $\phi_{12}^{q}=\arg \left(-M_{12}^{q} / \Gamma_{12}^{q}\right)$. For our numerical analysis we use results for $\Gamma_{12,3}^{q,(0)}$, $\Gamma_{12,3}^{q,(1)}$ and $\Gamma_{12,4}^{q,(0)}$ from $[67,80,83-87]$, results for $M_{12}^{q}$ from [88, 89] and for the hadronic matrix elements of dimension six the averages presented in [65] based on [82, 90, 91] and [92-95]. Recently also the first non-perturbative evaluation of dimension seven matrix elements became available [96], which we will use for $\Gamma_{12}^{q}$. 


\subsection{QCD factorization}

In our analysis we included different observables based on non-leptonic $B$ meson decays such as: $B \rightarrow D \pi, B \rightarrow \pi \pi, B \rightarrow \pi \rho$ and $B \rightarrow \rho \rho$. To calculate the corresponding amplitudes we used the expressions available in the literature obtained within the QCD Factorization (QCDF) framework [17-20]. In this section we briefly summarise the QCDF results relevant for the evaluation of some of our flavour constraints. Consider the process $B \rightarrow M_{1} M_{2}$, in which a $B$ meson decays into the final states $M_{1}$ and $M_{2}$, where either $M_{1}$ and $M_{2}$ are two "light" mesons or $M_{1}$ is "heavy" and $M_{2}$ is "light". 3

If both $M_{1}$ and $M_{2}$ are light, then the matrix element $\left\langle M_{1} M_{2}\left|\hat{Q}_{i}\right| B\right\rangle$ of the dimension six effective operators in eq. (2.3) can be written as

$$
\begin{aligned}
\left\langle M_{1} M_{2}\left|\hat{Q}_{i}\right| B\right\rangle= & \sum_{j} F_{j}^{B \rightarrow M_{1}}(0) \int_{0}^{1} d u T_{i j}^{I}(u) \Phi_{M_{2}}(u)+\left(M_{1} \leftrightarrow M_{2}\right) \\
& +\int_{0}^{1} d \xi d u d v T_{i}^{I I}(\xi, u, v) \Phi_{B}(\xi) \Phi_{M_{1}}(v) \Phi_{M_{2}}(u) .
\end{aligned}
$$

In the right hand side of eq. (2.18) $F_{j}^{B \rightarrow M_{1,2}}\left(m_{2,1}^{2}\right)$ represents the relevant form factor to account for the transition $B \rightarrow M_{1}$ (and correspondingly for $B \rightarrow M_{2}$ ) and $\Phi_{M}(u)$ is the nonperturbative Light-Cone Distribution Amplitude (LCDA) for the meson $M$, see figure 1 .

Notice that eq. (2.18) is written in such a way that it can be applied to situations where the spectator quark can end in any of the two final state light mesons. If the spectator can go into only one of the final mesons, this one will be labelled as $M_{1}$ and just the first and the third terms on the right hand side of eq. (2.18) should be included. The functions $T^{I, I I}$ are called hard-scattering kernels and can be calculated perturbatively. The kernel $T^{I}$ contains, at higher order in $\alpha_{s}$, nonfactorizable contributions from hard gluon exchange or penguin topologies. On the other hand, nonfactorizable hard interactions involving the spectator quark are part of $T^{I I}$.

When in the final state the mesons $M_{1}$ is "heavy" and $M_{2}$ is "light", then the corresponding QCDF formula for the matrix element $\left\langle M_{1} M_{2}\left|\hat{Q}_{i}\right| B\right\rangle$ becomes

$$
\left\langle M_{1} M_{2}\left|\hat{Q}_{i}\right| B\right\rangle=\sum_{j} F_{j}^{B \rightarrow M_{1}}\left(m_{2}^{2}\right) \int_{0}^{1} d u T_{i j}^{I}(u) \Phi_{M_{2}}(u),
$$

where the meaning of the different terms in eq. (2.19) are analogous to those given for eq. (2.18).

To determine the decay amplitude $\mathcal{A}\left(B \rightarrow M_{1} M_{2}\right)$, the matrix element $\left\langle M_{1} M_{2}\left|\hat{\mathcal{H}}_{\text {eff }}\right| B\right\rangle$ should be calculated, with $\hat{\mathcal{H}}_{\text {eff }}$ being the effective Hamiltonian introduced in eq. (2.1). In QCDF the final expression for $\mathcal{A}\left(B \rightarrow M_{1} M_{2}\right)$ is written as a linear combination of subamplitudes $\alpha_{i}^{p, M_{1} M 2}$ and $\beta_{i}^{p, M_{1} M 2}$, which for the purposes of our discussion will be termed "Topological Amplitudes" (TA). The TA $\alpha_{i}^{p}\left(M_{1} M_{2}\right)$, for $p=u, c$, have the following

\footnotetext{
${ }^{3} \mathrm{~A}$ meson with mass $m$ is considered "heavy" if $m$ scales with $m_{b}$ in the heavy quark limit such that $m / m_{b}$ remains fixed. On the other hand a meson is regarded as "light" if its mass remains finite in the heavy quark limit, for a light meson $m \sim \mathcal{O}\left(\Lambda_{\mathrm{QCD}}\right)[18]$.
} 

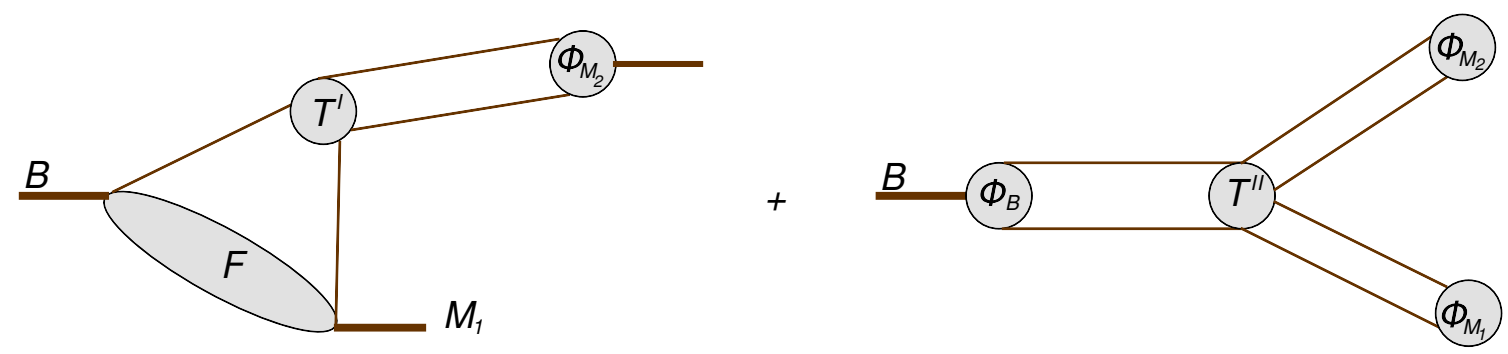

Figure 1. Factorization of matrix elements for B meson decays into "light"- "light" mesons (both diagrams included) and "heavy"-"light" (only left diagram) in QCDF.

generic structure at NLO in $\alpha_{s}[20]$

$$
\begin{aligned}
\alpha_{i}^{p, M_{1} M_{2}}= & {\left[C_{i}\left(\mu_{b}\right)+\frac{C_{i \pm 1}\left(\mu_{b}\right)}{N_{c}}\right] N_{i}\left(M_{2}\right) } \\
& +\frac{\alpha_{s}\left(\mu_{b}\right)}{4 \pi} \frac{C_{F}}{N_{c}} C_{i \pm 1}\left(\mu_{b}\right) V_{i}\left(M_{2}\right)+P_{i}^{p}\left(M_{2}\right) \\
& +\frac{\alpha_{s}\left(\mu_{h}\right)}{4 \pi} \frac{4 \pi^{2} C_{F}}{N_{c}^{2}} C_{i \pm 1}\left(\mu_{h}\right) H_{i}\left(M_{1} M_{2}\right)
\end{aligned}
$$

where $C_{i}$ are the Wilson coefficients calculated at the scale $\mu \sim m_{b}$, and the subindex in the coefficient $C_{i \pm 1}$ is assigned following the rule

$$
C_{i \pm 1}=\left\{\begin{array}{l}
C_{i+1}: \text { if } i \text { is odd } \\
C_{i-1}: \text { if } i \text { is even }
\end{array}\right.
$$

The Wilson coefficients inside the squared bracket in eq. (2.20) will be modified to allow for NP contributions as discussed below, see section 3 , and $N_{c}$ denotes the number of colours under consideration and will be taken as $N_{c}=3$. The global factor $N_{i}\left(M_{2}\right)$ multiplying the square bracket corresponds to the normalisation of the light cone distribution for the meson $M_{2}$, and is evaluated according to the following rule

$$
N_{i}\left(M_{2}\right)=\left\{\begin{array}{lr}
0: \text { if } i=6,8 \text { and } M_{2} & \text { is a vector meson } \\
1: & \text { in any other case }
\end{array}\right.
$$

The symbol $V_{i}\left(M_{2}\right)$ in eq. (2.20) stands for the one loop vertex corrections illustrated in figure 2. Additionally, the contributions from Penguin diagrams such as those shown in figure 3 are included in $P_{i}^{p}\left(M_{2}\right)$, with $p=u, c$. Finally the hard spectator interactions shown in figure 4 are accounted for by the term $H_{i}\left(M_{1} M_{2}\right)$. If $M_{1}$ and $M_{2}$ are both pseudoscalar mesons or if one of them is a pseudoscalar and the other is a vector meson, then the hard spectator function $H_{i}\left(M_{1} M_{2}\right)$ can be written in terms of the leading twist LCDAs 

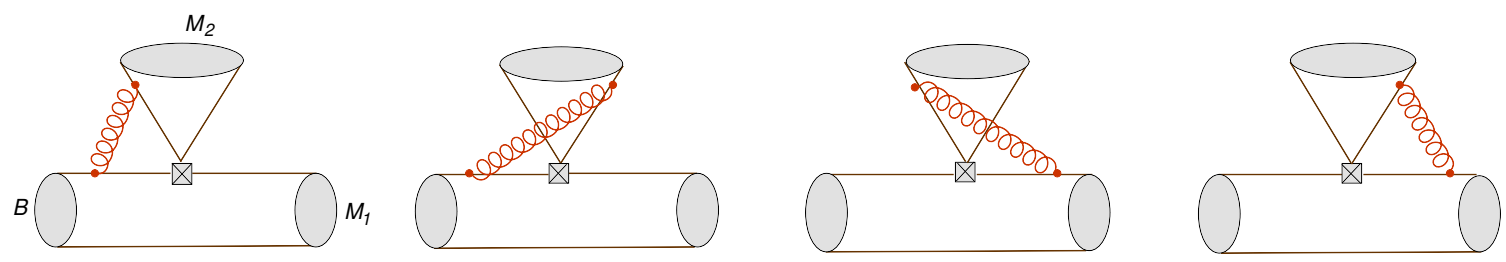

Figure 2. NLO Vertex contributions to the process $B \rightarrow M_{1} M_{2}$.
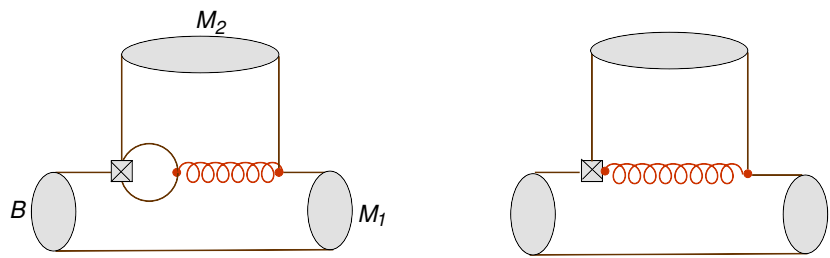

Figure 3. NLO penguin contributions to the process $B \rightarrow M_{1} M_{2}$.
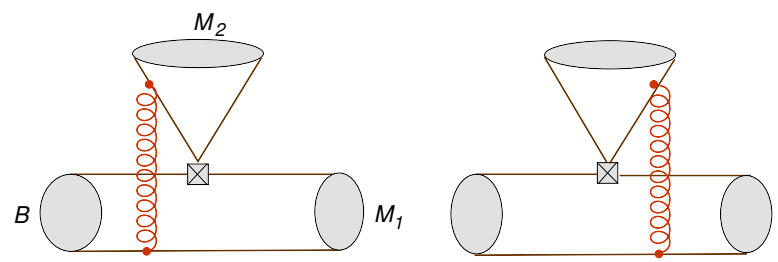

Figure 4. Hard spectator-scattering contributions to the decay $B \rightarrow M_{1} M_{2}$.

of $M_{1}$ and $M_{2}, \Phi_{M_{1}}$ and $\Phi_{M_{2}}$ respectively, and the twist-3 LCDA of $M_{1}, \Phi_{m_{1}}$, as [20]:

$$
\begin{aligned}
H_{i}\left(M_{1} M_{2}\right)= & \frac{B_{M_{1} M_{2}}}{A_{M_{1} M_{2}}} \int_{0}^{1} d \xi \frac{\Phi_{B}(\xi)}{\xi} \int_{0}^{1} d x \int_{0}^{1} d y\left[\frac{\Phi_{M_{2}}(x) \Phi_{M_{1}}(y)}{\bar{x} \bar{y}}\right. \\
& \left.+r_{\chi}^{M_{1}} \frac{\Phi_{M_{2}}(x) \Phi_{m_{1}}(y)}{x \bar{y}}\right],(\text { for } i=1, \ldots, 4,9,10), \\
H_{i}\left(M_{1} M_{2}\right)= & -\frac{B_{M_{1} M_{2}}}{A_{M_{1} M_{2}}} \int_{0}^{1} d \xi \frac{\Phi_{B}(\xi)}{\xi} \int_{0}^{1} d x \int_{0}^{1} d y\left[\frac{\Phi_{M_{2}}(x) \Phi_{M_{1}}(y)}{x \bar{y}}\right. \\
& \left.+r_{\chi}^{M_{1}} \frac{\Phi_{M_{2}}(x) \Phi_{m_{1}}(y)}{\bar{x} \bar{y}}\right],(\text { for } i=5,7), \\
H_{i}\left(M_{1} M_{2}\right)= & 0,(\text { for } i=6,8) .
\end{aligned}
$$

The analogous expressions for $H_{i}\left(M_{1} M_{2}\right)$ when $M_{1}$ and $M_{2}$ are two longitudinally polarised light vector mesons can be found in [97, 98]. We provide the functions $H_{i}\left(M_{1} M_{2}\right)$ for the processes relevant to this project in appendix B. The global coefficients $A_{M_{1} M_{2}}$ and $B_{M_{1} M_{2}}$ presented in eqs. (2.21) depend on form factors and decay constants and are given in eq. (B.1) also in appendix B.

We want to highlight two sources of uncertainty arising in eq. (2.21). The first one stems from the contribution of the twist-3 LCDA $\Phi_{m_{1}}(y)$. Since this function does not 
vanish at $y=1$, the integral $\int_{0}^{1} d y \Phi_{m_{1}}(y) / \bar{y}$ is divergent. To isolate the divergence we follow the prescription given in [20] and write

$$
\begin{aligned}
\int_{0}^{1} \frac{d y}{\bar{y}} \Phi_{m_{1}}(y) & =\Phi_{m_{1}}(1) \int_{0}^{1} \frac{d y}{\bar{y}}+\int_{0}^{1} \frac{d y}{\bar{y}}\left[\Phi_{m_{1}}(y)-\Phi_{m_{1}}(1)\right] \\
& =\Phi_{m_{1}}(1) X_{H}+\int_{0}^{1} \frac{d y}{[\bar{y}]_{+}} \Phi_{m_{1}}(y) .
\end{aligned}
$$

The divergent piece of eq. (2.22) is contained in $X_{H}$. The remaining integral $\int_{0}^{1} d y /[\bar{y}]_{+} \Phi_{m_{1}}(y)$ is finite (for instance for a pseudo scalar meson $\Phi_{m_{1}}(y)=1$ and trivially $\left.\int_{0}^{1} d y /[\bar{y}]_{+} \Phi_{m_{1}}(y)=0\right)$. Physically $X_{H}$ represents a soft gluon interaction with the spectator quark. It is expected that $X_{H} \approx \ln \left(m_{b} / \Lambda_{\mathrm{QCD}}\right)$ because the divergence appearing is regulated by a physical scale of the order $\Lambda_{\mathrm{QCD}}$. A complex coefficient cannot be excluded since multiple soft scattering can introduce a strong interaction phase. Here we use the standard parameterisation for $X_{H}$ introduced by Beneke-Buchalla-Neubert-Sachrajda (BBNS) [18]

$$
X_{H}=\left(1+\rho_{H} e^{i \phi_{H}}\right) \ln \frac{m_{B}}{\Lambda_{h}},
$$

where $\Lambda_{h} \approx \mathcal{O}\left(\Lambda_{\mathrm{QCD}}\right)$ and $\rho_{H} \approx \mathcal{O}(1)$.

The second source of theoretical uncertainty in eqs. (2.21) that deserves special attention is the inverse moment of the LCDA $\Phi_{B}$ corresponding to the $B$ meson. Following [17] we write

$$
\int_{0}^{1} d \xi \frac{\Phi_{B}(\xi)}{\xi} \equiv \frac{m_{B}}{\lambda_{B}}
$$

where $\lambda_{B}$ is expected to be of $\mathcal{O}\left(\Lambda_{\mathrm{QCD}}\right)$. We provide more details about the values for $X_{H}$ and $\lambda_{B}$ used in this work at the end of this subsection.

Next we address the contributions from weak annihilation topologies, see figure 5 , which are power suppressed in the $\Lambda_{\mathrm{QCD}} / m_{b}$ expansion with respect to the factorizable amplitudes. Although they do not appear in eq. (2.18), they are included in terms of subamplitudes denoted as $\beta_{k}^{p, M_{1} M_{2}}$. The numerical subscript $k$ describes the Dirac structure under consideration: $k=1$ for $(V-A) \otimes(V-A), k=2$ for $(V-A) \otimes(V+A)$ and $k=3$ for $(-2)(S-P) \otimes(S+P)$. The annihilation coefficients are expressed in terms of a set of basic "building blocks" denoted by $A_{k}^{i, f}$. Where the subindex $k$ also denotes the Dirac structure being considered as previously explained, and the superindices $i$ and $f$ denote the emission of a gluon by an initial or a final state quark as shown in figure 5 . The coefficients $A_{k}^{i, f}$ relevant for this work can be found in appendix B. The final expressions for annihilation are the result of the convolution of twist-2 and twist-3 LCDA with the corresponding hard scattering kernels; as in the case of hard spectator scattering, there are also endpoint singularities that are treated in a model dependent fashion. To parameterize these divergences, we follow once more the approach of BBNS. Thus, in analogy with hard spectator scattering we introduce [18]

$$
X_{A}=\left(1+\rho_{A} e^{i \phi_{A}}\right) \ln \frac{m_{B}}{\Lambda_{h}}
$$



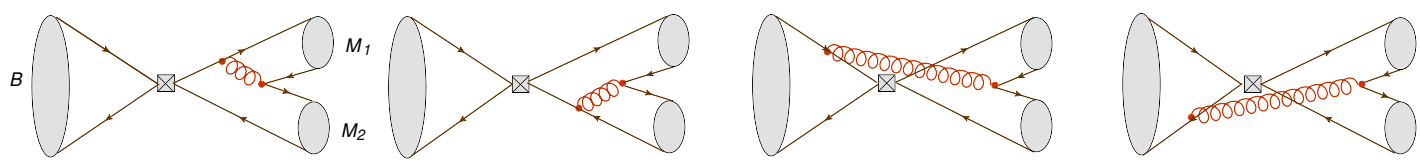

Figure 5. Annihilation topologies contributing to the decay process $B \rightarrow M_{1} M_{2}$.

To finalize this subsection we discuss the numerical inputs used in our evaluations of $\lambda_{B}$, $X_{H}$ and $X_{A}$. As indicated in eq. (2.24), the inverse moment of the LCDA of the $B$ meson introduces the parameter $\lambda_{B}$. The description of non-leptonic $B$ decays based on QCDF requires $\lambda_{B} \sim 200 \mathrm{MeV}[20,99]$. In contrast, QCD sum rules calculations give a higher value. For instance, in [100] the result $\lambda_{B}=(460 \pm 110) \mathrm{MeV}$ was found. In [101] the usage of the channel $B \rightarrow \gamma \ell \nu_{\ell}$ was proposed in order to extract $\lambda_{B}$ experimentally. This study was updated in [102-104] where further effects, including subleading power corrections in $1 / E_{\gamma}$ and $1 / m_{b}$, were accounted for. Based on this idea, the Belle collaboration found [105]

$$
\left.\lambda_{B}\right|_{\text {Belle }}>238 \mathrm{MeV}
$$

at the $90 \%$ C.L. and it is expected that the Belle II experiment improves this result [103]. Interestingly the experimental bound in eq. (2.26) is compatible with the QCD sum rules value quoted above and other theoretical approaches, including the one in [106] where the value $\lambda_{B}=(476.19 \pm 113.38) \mathrm{MeV}$ was obtained. For the purposes of our analysis, we consider the following result calculated in [107] with QCD sum rules:

$$
\lambda_{B}=(400 \pm 150) \mathrm{MeV} .
$$

As discussed above, the calculation of hard spectator interactions and the evaluation of annihilation topologies, leads to extra sources of uncertainty associated with endpoint singularities that are power suppressed. As indicated in eqs. (2.23) and (2.25) they can be parameterized through the functions $X_{H}\left(\rho_{H}, \phi_{H}\right)$ and $X_{A}\left(\rho_{A}, \phi_{A}\right)$ respectively. Using these models, we account for the hard spectator scattering power suppressed singularities through the parameters $\rho_{H}$ and $\phi_{H}$. Correspondingly, we introduce $\rho_{A}$ and $\phi_{A}$ to address the analogous effects from annihilation topologies. Based on phenomenological considerations we will take into account the intervals $[56,108]$

$$
0<\rho_{H, A}<2,0<\phi_{H, A}<2 \pi,
$$

which correspond to a $200 \%$ uncertainty on $\left|X_{H}\right|$ and $\left|X_{A}\right|$.

To evaluate the central values of our observables we take $\rho_{H, A}=0$, or equivalently $X_{H}=X_{A}=\ln m_{B} / \Lambda_{h}$. Finally, we calculate the percentual error from $X_{A}$ and $X_{H}$, by estimating the difference between the maximum and the minimum values reached by the hadronic observables when considering the intervals in eq. (2.28), and then we normalize by two times the corresponding central values.

\section{Strategy}

Consider the effective Hamiltonian in eq. (2.1) written in terms of the basis in eq. (2.3). We introduce "new physics" in the Wilson coefficients $\left\{C_{1}, C_{2}\right\}$ of the operators $\hat{Q}_{1}$ and 
$\hat{Q}_{2}$ following the prescription

$$
\begin{aligned}
& C_{1}\left(M_{W}\right):=C_{1}^{\mathrm{SM}}\left(M_{W}\right)+\Delta C_{1}\left(M_{W}\right), \\
& C_{2}\left(M_{W}\right):=C_{2}^{\mathrm{SM}}\left(M_{W}\right)+\Delta C_{2}\left(M_{W}\right),
\end{aligned}
$$

where in the SM

$$
\begin{aligned}
& \Delta C_{1}\left(M_{W}\right)=0, \\
& \Delta C_{2}\left(M_{W}\right)=0 .
\end{aligned}
$$

In this paper we present possible bounds on $\Delta C_{1}$ and $\Delta C_{2}$ at the matching scale $\mu=M_{W}$ and consider changes to each Wilson coefficient independently, e.g. to establish constraints on $\Delta C_{1}\left(M_{W}\right)$ we fix $\Delta C_{2}\left(M_{W}\right)=0$ and vice versa. This is a conservative approach, if we allow both parameters to change simultaneously this can result into partial cancellations leading to potentially bigger NP allowed regions for $\left\{\Delta C_{1}\left(M_{W}\right), \Delta C_{2}\left(M_{W}\right)\right\}$. Since the theoretical formulae for our observables are calculated at the scale $\mu=m_{b}$, we evolve down the modified Wilson coefficients $C_{1}\left(M_{W}\right)$ and $C_{2}\left(M_{W}\right)$ up to this scale using the renormalisation group formalism described in section 2.1. We consider NP to be leading order only, therefore we treat the SM contribution $\left\{C_{1}^{\mathrm{SM}}\left(M_{W}\right), C_{2}^{\mathrm{SM}}\left(M_{W}\right)\right\}$ and the NP components $\left\{\Delta C_{1}\left(M_{W}\right), \Delta C_{2}\left(M_{W}\right)\right\}$ differently under the renormalisation group equations. For instance the evolution of $\left\{C_{1}^{\mathrm{SM}}\left(M_{W}\right), C_{2}^{\mathrm{SM}}\left(M_{W}\right)\right\}$ is done using the full NLO expressions in eq. (2.5), on the other hand $\left\{\Delta C_{1}\left(M_{W}\right), \Delta C_{2}\left(M_{W}\right)\right\}$ are evolved down using only the LO version shown in eq. (2.7). Notice that, even though at the scale $\mu=M_{W}$ the only modified Wilson coefficients are $C_{1}\left(M_{W}\right)$ and $C_{2}\left(M_{W}\right)$, the non diagonal nature of the evolution matrices propagates these effects to all the other Wilson coefficients undergoing mixing at $\mu=m_{b}$. Hence, when writing expressions for the different physical observables, it makes sense to consider NP effects in $C_{i}\left(m_{b}\right)$ even for $i \neq 1,2$.

\subsection{Statistical analysis}

The values of $\Delta C_{1}\left(M_{W}\right)$ and $\Delta C_{2}\left(M_{W}\right)$ compatible with experimental data are evaluated using the program MyFitter [63]. The full statistical procedure is based on a likelihood ratio test. The basic ingredient is the $\chi^{2}$ function

$$
\chi^{2}(\vec{\omega})=\sum_{i}\left(\frac{\tilde{O}_{i, \exp }-\tilde{O}_{i, \text { theo }}(\vec{\omega})}{\sigma_{i, \exp }}\right)^{2},
$$

where $\tilde{O}_{i, \exp }$ and $\tilde{O}_{i, \text { theo }}$ are the experimental and theoretical values of the $i$-th observable respectively and $\sigma_{i, \exp }$ is the corresponding experimental uncertainty. The vector $\vec{\omega}$ contains all the inputs necessary for the evaluation of $\tilde{O}_{i, \text { theo }}$ and will be written as

$$
\vec{\omega}=\left(\Delta C_{1}\left(M_{W}\right), \Delta C_{2}\left(M_{W}\right), \vec{\lambda}\right) .
$$

In eq. (3.4) we are making a distinction between $\left\{\Delta C_{1}\left(M_{W}\right), \Delta C_{2}\left(M_{W}\right)\right\}$ and the rest of the theoretical inputs, which have been included in the subvector $\vec{\lambda}$. Examples of the entries 
inside $\vec{\lambda}$ are masses, decay constants, form-factors, etc. . Notice that our main target is the determination of $\Delta C_{1}\left(M_{W}\right)$ and $\Delta C_{2}\left(M_{W}\right)$, however, the components entering $\vec{\lambda}$ are crucial in defining the uncertainty of our observables and hence in establishing the potential values of $\Delta C_{1}\left(M_{W}\right)$ and $\Delta C_{2}\left(M_{W}\right)$. In this respect, we will say that the elements inside $\vec{\lambda}$ are our nuisance parameters, and that the determination of the possible NP values compatible with data are obtained by profiling the likelihood with respect to $\left\{\Delta C_{1}\left(M_{W}\right), \Delta C_{2}\left(M_{W}\right)\right\}$. During our analysis the elements of $\left\{\Delta C_{1}\left(M_{W}\right), \Delta C_{2}\left(M_{W}\right)\right\}$ are assumed to be complex and, as indicated in the argument, the initial evaluation is done at the scale $\mu=M_{W}$. The statistical theory behind the $\chi^{2}$-fit software used, e.g. MyFitter [63], can be found in the documentation of the computer program. Here we only summarize the key steps involved in our analysis:

1. We first define the Confidence Level $C L$ for the $\chi^{2}$-fit. Following the criteria established in $[55,57]$ for our study we take

$$
C L=90 \%
$$

which is equivalent to 1.64 standard deviations approximately.

2. Then, we establish a sampling region on the plane defined by the real and the imaginary components of $\left\{\Delta C_{1}\left(M_{W}\right), \Delta C_{2}\left(M_{W}\right)\right\}$. The sampling region is observable dependent. In our case we opt for rectangular grids around the origin of the complex plane defined by $\Delta C_{1}\left(M_{W}\right)$ and $\Delta C_{2}\left(M_{W}\right)$. Notice that the origin of our complex plane corresponds to the SM value. The number of points in our test grid depends on three factors: the numerical stability of our algorithms, on the time required to compute a particular combination of observables and the size of the NP regions determined by them.

3. Each one of the points inside the sampling grid described in the previous step corresponds to a null-hypothesis for the components of $\Delta C_{1}\left(M_{W}\right)$ and $\Delta C_{2}\left(M_{W}\right)$. We test our null-hypothesis values using a likelihood ratio test considering the confidence level established in the first step. For a combination of multiple observables several nuisance parameters are involved and the full statistical procedure becomes time and resource consuming. Hence, the parallelization of our calculations using a computer cluster became necessary. We did our first numerical evaluations partially at the Institute for Particle Physics and Phenomenology (IPPP, Durham University). The results presented in this work were obtained in full using the computing facilities available at the Dutch National Institute for Subatomic Physics (Nikhef).

\section{Individual constraints}

In this section we present the different observables considered during the analysis. From sections 4.1 to 4.3 we focus exclusively on observables that constrain individual $b$ decay channels, in our case: $b \rightarrow c \bar{u} d, b \rightarrow u \bar{u} d, b \rightarrow c \bar{c} s$ and $b \rightarrow c \bar{c} d$. In section 4.5 we will study observables that affect multiple $b$ decay channels. In what follows and unless stated 
otherwise, the SM predictions as well as the experimental determinations are given at $1 \sigma$, i.e. $68 \%$ C.L.. However the allowed NP regions for $C_{1}$ and $C_{2}$ are presented at $1.64 \sigma$, i.e. $90 \%$ C.L..

Following the notation introduced in eqs. (2.1) and (2.3) we will denote the NP effects in the Wilson coefficient of the operator $\hat{Q}_{i}^{q, p p^{\prime}}$ as $\Delta C_{i}^{q, p p^{\prime}}$ for $i=1,2$ and $q=d, s$. Then for example, $\left\{\Delta C_{1}^{d, c u}\left(M_{W}\right), \Delta C_{2}^{d, c u}\left(M_{W}\right)\right\}$ will quantify the potential deviations from the SM values in the coefficients of $\left\{\hat{Q}_{1}^{d, c u}, \hat{Q}_{2}^{d, c u}\right\}$ which describe the tree level process $b \rightarrow c \bar{u} d$.

In this work NP is supposed to be leading order in $\alpha_{s}$ and $\alpha$ only. Since all the vertex corrections $V_{i}^{M}$, penguins $P_{i}^{p, M}$ and hard scattering spectator interactions $H_{i}^{M_{1} M_{2}}$ inside eq. (2.20) are already suppressed by factors of $\mathcal{O}\left(\alpha_{s}\right)$ and $\mathcal{O}(\alpha)$, we will consistently drop the extra contributions $\Delta C_{1}^{d, u u}\left(M_{W}\right)$ and $\Delta C_{2}^{d, u u}\left(M_{W}\right)$ affecting any of these terms for all observables that are described by QCDF.

\subsection{Observables constraining $b \rightarrow c \bar{u} d$ transitions}

We start with the dominant quark level decay $b \rightarrow c \bar{u} d$ and describe our analysis of the potential NP regions for $\Delta C_{1}^{d, c u}\left(M_{W}\right)$ and $\Delta C_{2}^{d, c u}\left(M_{W}\right)$. The decay $\bar{B}^{0} \rightarrow D^{*+} \pi^{-}$will exclude large positive values of $\Delta C_{1}^{d, c u}\left(M_{W}\right)$ and it will significantly constrain $\Delta C_{2}^{d, c u}\left(M_{W}\right)$.

\subsection{1 $\quad \bar{B}_{d}^{0} \rightarrow D^{*+} \pi^{-}$}

Our bounds will be established using the ratio between the decay width for the non-leptonic decay $\bar{B}_{d}^{0} \rightarrow D^{*+} \pi^{-}$and the differential rate for the semi-leptonic process $\bar{B}_{d}^{0} \rightarrow D^{*+} l^{-} \bar{\nu}_{l}$ evaluated at $q^{2}=m_{\pi}^{2}$ for $l=e, \mu$

$$
R_{D^{*} \pi}=\frac{\Gamma\left(\bar{B}^{0} \rightarrow D^{*+} \pi^{-}\right)}{d \Gamma\left(\bar{B}^{0} \rightarrow D^{*+} l^{-} \bar{\nu}_{l}\right) /\left.d q^{2}\right|_{q^{2}=m_{\pi}^{2}}} \simeq 6 \pi^{2} f_{\pi}^{2}\left|V_{u d}\right|^{2}\left|\alpha_{2}^{D^{*} \pi}+\beta_{2}^{D^{*} \pi}\right|^{2} .
$$

This observable was proposed by Bjorken to test the factorization hypothesis [109], it is free from the uncertainties associated with the required form factor to describe the transition $B \rightarrow D^{*}$ and offers the possibility of comparing directly the coefficient $\alpha_{2}^{D^{*} \pi}$ calculated using QCDF against experimental observations. At NLO the TA $\alpha_{2}^{D^{*} \pi}$ [18] is given by

$$
\begin{aligned}
\alpha_{2}^{\mathrm{NLO}, D^{*} \pi}= & C_{2}^{d, c u}\left(\mu_{b}\right)+\frac{C_{1}^{d, c u}\left(\mu_{b}\right)}{3}+\frac{\alpha_{s}\left(\mu_{b}\right)}{4 \pi} \frac{C_{F}}{N_{c}} C_{1}^{d, c u}\left(\mu_{b}\right)\left[-\tilde{B}-6 \ln \frac{\mu^{2}}{m_{b}^{2}}\right. \\
& \left.+\int_{0}^{1} d u F\left(u,-x_{c}\right) \Phi_{\pi}(u)\right] \approx 1.057 \pm 0.040,
\end{aligned}
$$

where the term $\tilde{B}$ inside the square bracket cancels the renormalisation scheme dependence of the Wilson coefficients $C_{1}^{d, c u}$ and $C_{2}^{d, c u}$, which in naive dimensional regularisation requires $\tilde{B}=11$. The kernel $F\left(u,-x_{c}\right)$ includes $\mathrm{QCD}$ vertex corrections arising in the decay $b \rightarrow c \bar{u} d$ and has to be evaluated at $x_{c}=\bar{m}_{c}\left(\bar{m}_{b}\right) / \bar{m}_{b}$ before being convoluted with the light-cone distribution $\Phi_{\pi}$ associated with the $\pi^{-}$meson in the final state. For the explicit evaluation of eq. (4.1) we use the updated determination of the TA $\alpha_{2}^{D^{*} \pi}$ at NNLO calculated in [110]

$$
\left|\alpha_{2}^{\mathrm{NNLO}, D^{*} \pi}\right|=1.071_{-0.014}^{+0.013}
$$




\begin{tabular}{|c|c|}
\hline Parameter & Relative error \\
\hline$X_{A}$ & $13.05 \%$ \\
\hline$\mu$ & $2.53 \%$ \\
\hline$f_{\pi}$ & $1.23 \%$ \\
\hline$\Lambda_{5}^{\mathrm{QCD}}$ & $0.09 \%$ \\
\hline$A_{0}^{B \rightarrow D^{*}}$ & $0.08 \%$ \\
\hline$f_{B}$ & $0.02 \%$ \\
\hline Total & $13.35 \%$ \\
\hline
\end{tabular}

Table 1. Error budget for the observable $R_{D^{*} \pi}$.

The annihilation topologies contributions are taken into account through

$$
\beta_{2}^{D^{*} \pi}=\frac{C_{F}}{N_{c}^{2}} \frac{B_{D^{*} \pi}}{A_{D^{*} \pi}} C_{2}^{d, c u}\left(\mu_{h}\right) A_{1}^{i}\left(\mu_{h}\right) \approx 0.014 \pm 0.045,
$$

where

$$
\frac{B_{D^{*} \pi}}{A_{D^{*} \pi}}=\frac{f_{B} f_{D^{*}}}{m_{B}^{2} A_{0}^{B \rightarrow D^{*}}(0)},
$$

and

$$
A_{1}^{i}\left(\mu_{h}\right) \approx 6 \pi \alpha_{s}\left(\mu_{h}\right)\left[3\left(X_{A}-4+\frac{\pi^{2}}{3}\right)+r_{\chi}^{D^{*}}\left(\mu_{h}\right) r_{\chi}^{\pi}\left(\mu_{h}\right)\left(X_{A}^{2}-2 X_{A}\right)\right],
$$

with the parameters $X_{A}$ are given in eq. (2.25) and the factors $r_{\chi}^{\pi}$ and $r_{\chi}^{D^{*}}$ quoted in eq. (B.1). Using the numerical inputs given in $\mathrm{A}$ we find

$$
R_{D^{*} \pi}^{\mathrm{SM}}=(1.12 \pm 0.15) \mathrm{GeV}^{2}
$$

corresponding to $x_{c}=0.225$, the partial contributions to the total error are shown in table 1.

The SM result is dominated by the contribution of $C_{2}$, thus we will get from $R_{D^{*} \pi}$ strong constraints on $C_{2}$ and relatively weak ones on $C_{1}$. To compute the experimental result we use [110]

$$
d \Gamma\left(\bar{B}_{d}^{0} \rightarrow D^{*+} l^{-} \bar{\nu}_{l}\right) /\left.d q^{2}\right|_{q^{2}=m_{\pi}^{2}}=(2.04 \pm 0.10) \cdot 10^{-3} \mathrm{GeV}^{-2} \mathrm{ps}^{-1},
$$

together with $[111]$

$$
\mathcal{B} r\left(\bar{B}^{0} \rightarrow D^{*+} \pi^{-}\right)=(2.84 \pm 0.15) \cdot 10^{-3},
$$

to obtain

$$
R_{D^{*} \pi}^{\mathrm{Exp}}=(0.92 \pm 0.07) \mathrm{GeV}^{2} .
$$

Our $\chi^{2}$-fit provides the $90 \%$ confidence level regions allowed by $\Delta C_{1}^{d, c u}\left(M_{W}\right)$ and $\Delta C_{2}^{d, c u}\left(M_{W}\right)$ displayed in figure 6 , which show that $\Delta C_{1}^{d, c u}\left(M_{W}\right)$ is quite unconstrained. On the other hand, there are stronger restrictions on the values that $\Delta C_{2}^{d, c u}\left(M_{W}\right)$ can assume. This is not surprising considering that $C_{2}^{d, c u}$ gives the leading contribution to $\alpha_{2}^{D^{*} \pi}$; this can be seen in the NLO version of the formula for this term in eq. (4.2). 

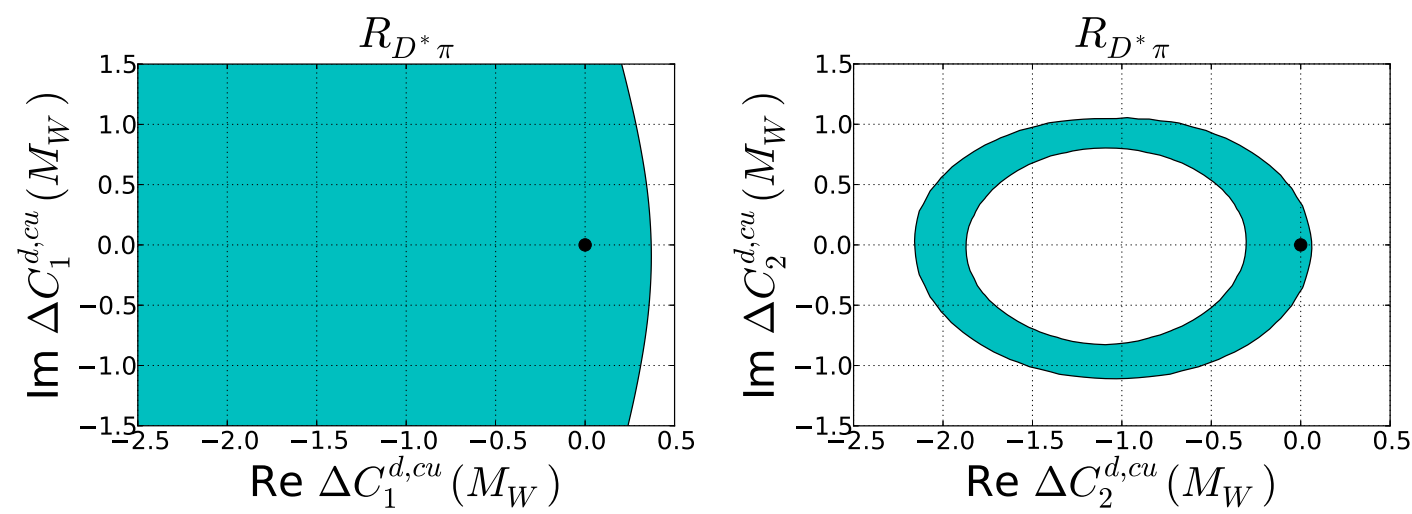

Figure 6. Potential regions for the NP contributions in $\Delta C_{1}^{d, c u}\left(M_{W}\right)$ and $\Delta C_{2}^{d, c u}\left(M_{W}\right)$ allowed by the observable $R_{D^{*} \pi}$ at $90 \%$ C.L. . The black point corresponds to the SM value. Since $R_{D^{*} \pi}$ is dominated by $C_{2}$, we get strong constraints on $C_{2}$ and relatively weak ones on $C_{1}$.

\subsection{Observables constraining $b \rightarrow u \bar{u} d$ transitions}

We proceed to describe the constraints to the NP contributions $\Delta C_{1,2}^{d, u u}\left(M_{W}\right)$ entering in the CKM suppressed quark level transition $b \rightarrow u \bar{u} d$. Our bounds are obtained taking into account both the branching ratios, but also the $\mathrm{CP}$ asymmetries of the decays $B \rightarrow \pi \pi, \rho \pi, \rho \rho$ and using again QCDF for the theoretical description. The combination of $\mathrm{CP}$-conserving and $\mathrm{CP}$-violating observables significantly shrinks the allowed region for $\Delta C_{2}^{d, u u}\left(M_{W}\right)$.

\subsection{1 $R_{\pi \pi}$}

Our first observable is the theoretical clean ratio [109]

$$
R_{\pi \pi}=\frac{\Gamma\left(B^{+} \rightarrow \pi^{+} \pi^{0}\right)}{d \Gamma\left(\bar{B}_{d}^{0} \rightarrow \pi^{+} \ell^{-} \bar{\nu}_{\ell}\right) /\left.d q^{2}\right|_{q^{2}=0}} \simeq 3 \pi^{2} f_{\pi}^{2}\left|V_{u d}\right|^{2}\left|\alpha_{1}^{\pi \pi}+\alpha_{2}^{\pi \pi}\right|^{2},
$$

where $\ell^{-}=\mu^{-}, e^{-}$and $\alpha_{1}^{\pi \pi}, \alpha_{2}^{\pi \pi}$ are the TA associated with the decays $B \rightarrow \pi \pi$ which were introduced in a generic way in eq. (2.20). The dependence of $R_{\pi \pi}$ is now symmetric in $C_{1}$ and $C_{2}$, so both Wilson coefficients will be constrained in an almost identical way. Notice that the denominator in eq. (4.11) refers to the differential distribution $d \Gamma\left(\bar{B}_{d}^{0} \rightarrow\right.$ $\left.\pi^{+} \ell^{-} \bar{\nu}_{\ell}\right) / d q^{2}$ evaluated at $q^{2}=0$, where $q^{2}$ is the four momentum transferred to the system composed by the $\ell^{-}$and $\bar{\nu}_{\ell}$. In eq. (4.11), our sensitivity to NP enters through the decay $B^{+} \rightarrow \pi^{+} \pi^{0}$ which is to a good degree of precision a pure tree level channel. We neglect hypothetical BSM effects in $\bar{B}_{d}^{0} \rightarrow \pi^{+} \ell^{-} \bar{\nu}_{\ell}$ for $\ell=e, \mu$, see e.g. [112] for a recent investigation of such a possibility. The observable $R_{\pi \pi}$ is theoretically clean since it does not depend on the CKM matrix element $\left|V_{u b}\right|$, which cancels in the ratio. Moreover, at leading order in $\alpha_{s}$ it is independent of the form factors $F_{+}^{B \rightarrow \pi}(0)=F_{0}^{B \rightarrow \pi}(0)$ which account for the hadronic transition $B \rightarrow \pi$. However, these parameters enter in the coefficients $\alpha_{1,2}^{\pi \pi}$ once the spectator interaction contributions $H_{\pi \pi}$ are taken into account. More precisely, they appear in the ratio $B_{\pi \pi} / A_{\pi \pi}$ inside $H_{\pi \pi}$, see eqs. (B.1) and (B.14). Currently, the coefficients $\alpha_{1,2}^{\pi \pi}$ in eq. (4.11) are available up to NNLO in QCDF [99, 113-115]. In order 
to optimize the computation time of our $\chi^{2}$-fit, we have accounted for the NNLO effects using the following formula

$$
\frac{\alpha_{1,2}^{\pi \pi}}{\alpha_{1,2}^{\mathrm{NNO}, \pi \pi}}=\frac{\alpha_{1,2}^{\mathrm{NLO}, \pi \pi}\left(\mu_{0}\right)}{\alpha_{1,2}^{(0) \mathrm{NLO}, \pi \pi}} .
$$

Where in eq. (4.12):

- $\alpha_{1,2}^{\mathrm{NLO}, \pi \pi}\left(\mu_{0}\right)$ corresponds to the fully programmed NLO expression for the amplitude $\alpha_{1,2}^{\pi \pi}$. For this term, the renormalization scale is kept fixed to the value $\mu_{0}=m_{b}$ whereas the rest of the input parameters are allowed to float.

- $\alpha_{1,2}^{(0)} \mathrm{NLO}, \pi \pi$ are the NLO version of the amplitudes $\alpha_{1,2}^{\pi \pi}$ evaluated at the central value of all the input parameters and kept constant during the $\chi^{2}$-fit.

- $\alpha_{1,2}^{\mathrm{NNLO}, \pi \pi}$ are the NNLO version of the amplitude $\alpha_{1,2}^{\pi \pi}$. We are interested in the NNLO results because of the reduction in the renormalisation scale dependency with respect to the NLO determination. Therefore during the $\chi^{2}$-fit we have treated the coefficients $\alpha_{1,2}^{\mathrm{NNLO}, \pi \pi}$ as nuisance parameters given by [107]

$$
\begin{aligned}
& \alpha_{1}^{\mathrm{NNLO}, \pi \pi}=0.195_{-0.025}^{+0.025}-\left(0.101_{-0.029}^{+0.021}\right) i, \\
& \alpha_{2}^{\mathrm{NNLO}, \pi \pi}=1.013_{-0.011}^{+0.008}+\left(0.027_{-0.013}^{+0.020}\right) i,
\end{aligned}
$$

where the error indicated arises only from the renormalization scale uncertainty. Alternatively, we also tested the numerical values provided in [99] which give consistent results once the uncertainties arising by varying $\mu$ and $\mu_{h},{ }^{4}$ are taken into account.

We predict the SM value of $R_{\pi \pi}$ to be

$$
R_{\pi \pi}^{\mathrm{SM}}=(0.70 \pm 0.14),
$$

with the partial contributions to the total error shown in table 2. To calculate the experimental result, we consider the following updated value for the branching fraction for the process $B^{+} \rightarrow \pi^{+} \pi^{0}[116]$

$$
\mathcal{B} r\left(B^{+} \rightarrow \pi^{+} \pi^{0}\right)=(5.5 \pm 0.4) \cdot 10^{-6},
$$

together with the product [117]

$$
\left|V_{u b} F_{+}^{B \rightarrow \pi}(0)\right|=(9.25 \pm 0.31) \cdot 10^{-4},
$$

which was extracted via a fit to data including experimental results from BaBar, Belle and CLEO [118-122] under the assumption of the SM, neglecting the mass of the light leptons and keeping the mass of the $B^{*}$ meson fixed. Using the inputs indicated in eqs. (4.15) and (4.16) we obtain the following result for the experimental value of $R_{\pi \pi}$

$$
R_{\pi \pi}^{\mathrm{Exp}}=(0.83 \pm 0.08) .
$$

\footnotetext{
${ }^{4}$ T. Huber, private communication.
} 


\begin{tabular}{|c|c|}
\hline Parameter & Relative Error \\
\hline$X_{H}$ & $16.86 \%$ \\
\hline$\lambda_{B}$ & $8.85 \%$ \\
\hline$\mu$ & $4.42 \%$ \\
\hline$a_{2}^{\pi}$ & $2.57 \%$ \\
\hline$F_{+}^{B \rightarrow \pi}(0)$ & $1.77 \%$ \\
\hline$f_{\pi}$ & $1.35 \%$ \\
\hline$m_{s}$ & $0.68 \%$ \\
\hline$\Lambda_{5}^{\mathrm{QCD}}$ & $0.25 \%$ \\
\hline$f_{B}$ & $0.14 \%$ \\
\hline$m_{b}$ & $0.04 \%$ \\
\hline$V_{u s}$ & $0.01 \%$ \\
\hline Total & $19.86 \%$ \\
\hline
\end{tabular}

Table 2. Error budget for the observable $R_{\pi \pi}$. Here $X_{H}$ accounts for the endpoint singularities from hard scattering spectator interactions. $F_{+}^{B \rightarrow \pi}(0)$ is the relevant form factor for the transitions $B \rightarrow \pi$. The parameter $\lambda_{B}$ is the inverse moment of the LCDA of the $B$ meson and $a_{2}^{\pi}$ is the second Gegenbauer moment for the $\pi$ meson.
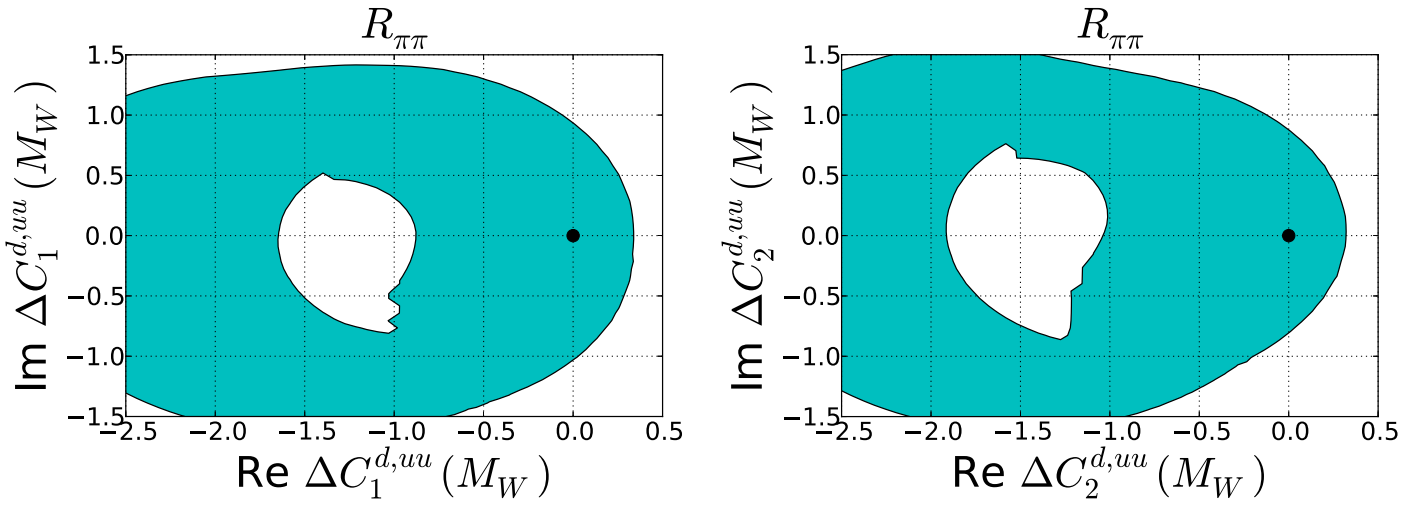

Figure 7. Potential regions for the NP contributions $\Delta C_{1}^{d, u u}\left(M_{W}\right)$ and $\Delta C_{2}^{d, u u}\left(M_{W}\right)$ allowed by the observable $R_{\pi \pi}$ at $90 \%$ C.L. . The black point corresponds to the SM value. The dependence of $R_{\pi \pi}$ is symmetric in $C_{1}$ and $C_{2}$, therefore both Wilson coefficients are constrained in an almost identical way.

This determination is in agreement with the result given in [99], however, the uncertainty is reduced by nearly $50 \%$ due to the update on the product $\left|V_{u b} F_{+}^{B \rightarrow \pi}(0)\right|$ shown in eq. (4.16). The allowed regions for $\Delta C_{1}^{d, u u}\left(M_{W}\right)$ and $\Delta C_{2}^{d, u u}\left(M_{W}\right)$ are shown in figure 7 - we note here rather stringent constraints on positive and real values of $\Delta C_{1}^{d, u u}\left(M_{W}\right)$ and $\Delta C_{2}^{d, u u}\left(M_{W}\right)$. 


\subsection{2 $S_{\pi \pi}$}

Since our NP contributions are allowed to be complex, we are exploring the possibility of having new $\mathrm{CP}$ violating phases. We can constrain these effects through the time-dependent asymmetries

$$
\begin{aligned}
\mathcal{A}_{f}^{C P}(t) & =\frac{d \Gamma\left[\bar{B}_{q}^{0} \rightarrow f\right](t) / d t-d \Gamma\left[B_{q}^{0} \rightarrow f\right](t) / d t}{d \Gamma\left[\bar{B}_{q}^{0} \rightarrow f\right](t) / d t+d \Gamma\left[B_{q}^{0} \rightarrow f\right](t) / d t} \\
& \simeq S_{f} \sin \Delta M_{q} t-C_{f} \cos \Delta M_{q} t,
\end{aligned}
$$

where we have neglected the effects of the observable $\Delta \Gamma_{q}$ entering in the denominator -

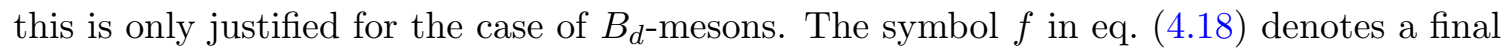
state to which both, the $B_{q}^{0}$ and the $\bar{B}_{q}^{0}$ meson can decay, for $q=d, s$. The mixing induced $\left(S_{f}\right)$ and direct $\mathrm{CP}$ asymmetries $\left(C_{f}\right)$ are defined as

$$
S_{f} \equiv \frac{2 \operatorname{Im}\left(\lambda_{f}^{q}\right)}{1+\left|\lambda_{f}^{q}\right|^{2}}, \quad C_{f} \equiv \frac{1-\left|\lambda_{f}^{q}\right|^{2}}{1+\left|\lambda_{f}^{q}\right|^{2}}
$$

with the parameter $\lambda_{f}^{q}$ given by

$$
\lambda_{f}^{q}:=\left.\frac{q}{p}\right|_{B_{q}} \frac{\bar{A}_{f}^{q}}{A_{f}^{q}} .
$$

In eq. (4.20) the amplitude for the process $B_{q}^{0} \rightarrow f$ has been denoted as $A_{f}^{q}$ and the one for $\bar{B}_{q}^{0} \rightarrow f$ as $\bar{A}_{f}^{q}$. Finally,

$$
\left.\frac{q}{p}\right|_{B_{q}}=\frac{M_{12}^{q *}}{\left|M_{12}^{q}\right|}
$$

where $M_{12}^{d}$ is the contribution from virtual internal particles to the $B_{q}^{0}-\bar{B}_{q}^{0}$ mixing diagrams. For instance in the case of $B_{d}$ mesons we get

$$
\left.\frac{q}{p}\right|_{B_{d}}=\left[\frac{V_{t d} V_{t b}^{*}}{\left|V_{t d} V_{t b}^{*}\right|}\right]^{2}
$$

Notice that the observable $S_{f}$, in eq. (4.19), is particularly sensitive to the imaginary components of $\Delta C_{1}\left(M_{W}\right)$ and $\Delta C_{2}\left(M_{W}\right)$.

For the decays $\bar{B}_{d}^{0} \rightarrow \pi^{+} \pi^{-}$and $B_{d}^{0} \rightarrow \pi^{+} \pi^{-}$we get

$$
S_{\pi \pi}=\frac{2 \operatorname{Im}\left(\lambda_{\pi \pi}^{d}\right)}{1+\left|\lambda_{\pi \pi}^{d}\right|^{2}}, \quad \lambda_{\pi \pi}^{d}=\left[\frac{V_{t d} V_{t b}^{*}}{\left|V_{t d} V_{t b}^{*}\right|}\right]^{2} \frac{\overline{\mathcal{A}}_{\pi^{+} \pi^{-}}}{\mathcal{A}_{\pi^{+} \pi^{-}}} .
$$

Here $\overline{\mathcal{A}}_{\pi^{+} \pi^{-}}$and $\mathcal{A}_{\pi^{+} \pi^{-}}$denote the transition amplitudes for the processes $\bar{B}_{d}^{0} \rightarrow \pi^{+} \pi^{-}$and $B_{d}^{0} \rightarrow \pi^{+} \pi^{-}$respectively. They have been calculated in [20] using the QCDF formalism briefly described in section 2.3. The explicit expression for $\overline{\mathcal{A}}_{\pi^{+} \pi^{-}}$is

$$
\begin{aligned}
\overline{\mathcal{A}}_{\pi^{+} \pi^{-}}= & A_{\pi \pi}\left(\lambda_{u}^{(d)} \alpha_{2}^{\pi \pi}+\lambda_{u}^{(d)} \beta_{2}^{\pi \pi}+\sum_{p=u, c} \lambda_{p}^{(d)}\left[\tilde{\alpha}_{4}^{p, \pi \pi}+\tilde{\alpha}_{4, E W}^{p, \pi \pi}\right.\right. \\
& \left.\left.+\beta_{3}^{p, \pi \pi}-1 / 2 \beta_{3, E W}^{p, \pi \pi}+2 \beta_{4}^{p, \pi \pi}+1 / 2 \beta_{4, E W}^{p, \pi \pi}\right]\right) .
\end{aligned}
$$

To determine the remaining amplitude $\mathcal{A}_{\pi^{+} \pi^{-}}$, the $\mathrm{CP}$ conjugate of the expression in eq. (4.24) has to be obtained. The parameters $\lambda_{u, c}^{(d)}$ in eq. (4.24) correspond to products of CKM matrix elements as defined in eq. (2.2). Notice that our sensitivity towards NP in tree 


\begin{tabular}{|c|c|}
\hline Parameter & Relative Error \\
\hline$X_{A}$ & $41.76 \%$ \\
\hline$\gamma$ & $6.24 \%$ \\
\hline$m_{s}$ & $4.43 \%$ \\
\hline$\left|V_{u b} / V_{c b}\right|$ & $4.31 \%$ \\
\hline$X_{H}$ & $3.08 \%$ \\
\hline$\mu$ & $2.79 \%$ \\
\hline$\Lambda_{5}^{\mathrm{QCD}}$ & $2.25 \%$ \\
\hline$\lambda_{B}$ & $1.55 \%$ \\
\hline$F_{+}^{B \rightarrow \pi}$ & $0.89 \%$ \\
\hline$m_{b}$ & $0.76 \%$ \\
\hline$\left|V_{u s}\right|$ & $0.13 \%$ \\
\hline$f_{B}$ & $0.07 \%$ \\
\hline$m_{c}$ & $0.06 \%$ \\
\hline$f_{\pi}$ & $0.06 \%$ \\
\hline$a_{2}^{\pi}$ & $0.03 \%$ \\
\hline Total & $42.98 \%$ \\
\hline
\end{tabular}

Table 3. Error budget for the observable $S_{\pi \pi}$. Most of the inputs coincide with those for $R_{\pi \pi}$ described in table 2. Additionally the effects of annihilation topologies are accounted by $X_{A}$.

level enters mainly through $\alpha_{2}^{\pi \pi}$, which according to eq. (2.20) has a leading dependency on $\Delta C_{2}^{d, u u}\left(M_{W}\right)$. Therefore, the observable $S_{\pi \pi}$ yields to strong constraints on $\Delta C_{2}^{d, u u}\left(M_{W}\right)$, while giving weak ones in $\Delta C_{1}^{d, u u}\left(M_{W}\right)$. Besides the TA $\alpha_{2}^{\pi \pi}$, which is introduced in our analysis at NNLO following the prescription shown in eq. (4.12), there are now also contributions from QCD and electroweak penguins given by ${\tilde{\alpha_{4}}}^{p, \pi \pi}$ and ${\tilde{\alpha_{4}}}_{E W}^{p, \pi \pi}$ respectively. Finally $\beta_{4}^{p, \pi \pi}$ accounts for QCD penguin annihilation and $\beta_{4, E W}^{p, \pi \pi}$ for electroweak penguin annihilation. All the TA can be calculated using eq. (2.20) together with the information presented in appendix B. At leading order in $\alpha_{s}$, the normalization factor $A_{\pi \pi}$ introduced in eq. (B.1), which depends on the form factor $F_{+}^{B \rightarrow \pi}(0)$ and the decay constant $f_{\pi}$, cancels in the ratio given in eq. (4.23). However it appears again once interactions with the spectator are taken into account. This leads to small effects in the error budget of $\mathcal{O}(1 \%)$ and $\mathcal{O}(0.1 \%)$ from $F_{+}^{B \rightarrow \pi}(0)$ and $f_{\pi}$ respectively, see table 3 . Our theoretical prediction for the SM value of the asymmetry $S_{\pi \pi}$ is

$$
S_{\pi \pi}^{\mathrm{SM}}=-0.59 \pm 0.25
$$

For the corresponding experimental value we have [111]

$$
S_{\pi \pi}^{\operatorname{Exp}}=-0.63 \pm 0.04,
$$

showing consistency with the SM estimation in eq. (4.25). The relevant constraints on $\Delta C_{2}^{d, u u}\left(M_{W}\right)$ derived from $S_{\pi \pi}$ are presented in figure $8-$ constraints on $\Delta C_{1}^{d, u u}\left(M_{W}\right)$ are very weak and will thus not be shown. 


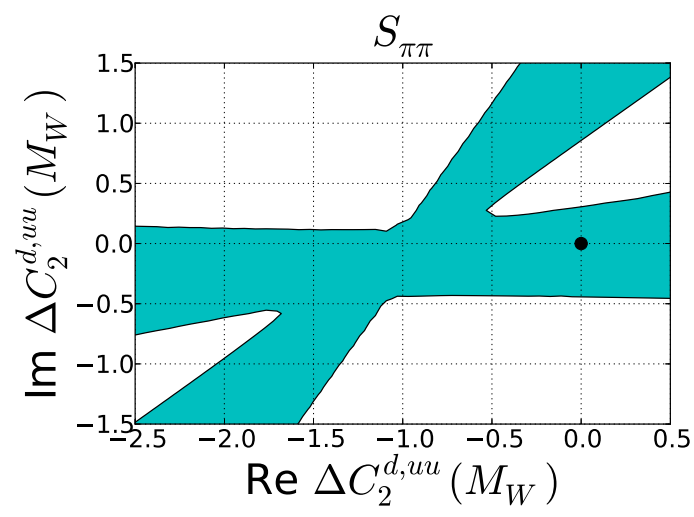

Figure 8. Potential regions for the NP contributions in $\Delta C_{2}^{d, u u}\left(M_{W}\right)$ allowed by the observable $S_{\pi \pi}$ at $90 \%$ C.L., the shift in the Wilson coefficient $\Delta C_{1}^{d, u u}\left(M_{W}\right)$ is only weakly constrained and therefore not shown. The black point corresponds to the SM value.

\subsection{3 $S_{\rho \pi}$}

We also included the mixing induced $\mathrm{CP}$ asymmetry associated with the decays $B_{d}, \bar{B}_{d} \rightarrow \rho \pi$. Our evaluation is based in the following definition

$$
S_{\pi \rho}=\frac{1}{2}\left(\tilde{S}_{\pi \rho}+\tilde{S}_{\rho \pi}\right)
$$

with the partial contributions given by

$$
\tilde{S}_{\pi \rho}=\frac{2 \operatorname{Im}\left(\lambda_{\pi \rho}^{d}\right)}{1+\left|\lambda_{\pi \rho}^{d}\right|^{2}}, \quad \tilde{S}_{\rho \pi}=\frac{2 \operatorname{Im}\left(\lambda_{\rho \pi}^{d}\right)}{1+\left|\lambda_{\rho \pi}^{d}\right|^{2}},
$$

with

$$
\lambda_{\pi \rho}^{d}=\left[\frac{V_{t d} V_{t b}^{*}}{\left|V_{t d} V_{t b}^{*}\right|}\right]^{2} \frac{\overline{\mathcal{A}}_{\pi^{+} \rho^{-}}}{\mathcal{A}_{\rho^{+} \pi^{-}}}, \quad \lambda_{\rho \pi}^{d}=\left[\frac{V_{t d} V_{t b}^{*}}{\left|V_{t d} V_{t b}^{*}\right|}\right]^{2} \frac{\overline{\mathcal{A}}_{\rho^{+} \pi^{-}}}{\mathcal{A}_{\pi^{+} \rho^{-}}} .
$$

The individual amplitudes $\overline{\mathcal{A}}_{\pi^{+} \rho^{-}}$and $\overline{\mathcal{A}}_{\rho^{+} \pi^{-}}$for the processes $\bar{B}_{d}^{0} \rightarrow \pi^{+} \rho^{-}$and $\bar{B}_{d}^{0} \rightarrow \rho^{+} \pi^{-}$ are respectively

$$
\begin{aligned}
\overline{\mathcal{A}}_{\pi^{+} \rho^{-}}= & A_{\pi \rho}\left(\lambda_{u}^{(d)} \alpha_{2}^{\pi \rho}+\sum_{p=u, c} \lambda_{p}^{(d)}\left[\tilde{\alpha}_{4}^{p, \pi \rho}+\tilde{\alpha}_{4, E W}^{p, \pi \rho}\right.\right. \\
& \left.\left.+\beta_{3}^{p, \pi \rho}+\beta_{4}^{p, \pi \rho}-\frac{1}{2} \beta_{3, E W}^{p, \pi \rho}-\frac{1}{2} \beta_{4, E W}^{p, \pi \rho}\right]\right) \\
& +A_{\rho \pi}\left(\lambda_{u}^{(d)} \beta_{1}^{\rho \pi}+\sum_{p=u, c} \lambda_{p}^{(d)}\left[\beta_{4}^{p, \rho \pi}+\beta_{4, E W}^{p, \rho \pi}\right]\right) \\
\overline{\mathcal{A}}_{\rho^{+} \pi^{-}}= & A_{\rho \pi}\left(\lambda_{u}^{(d)} \alpha_{2}^{\rho \pi}+\sum_{p=u, c} \lambda_{p}^{(d)}\left[\tilde{\alpha}_{4}^{p, \rho \pi}+\tilde{\alpha}_{4, E W}^{p, \rho \pi}+\beta_{3}^{p, \rho \pi}\right.\right. \\
& \left.\left.+\beta_{4}^{p, \rho \pi}-\frac{1}{2} \beta_{3, E W}^{p, \rho \pi}-\frac{1}{2} \beta_{4, E W}^{p, \rho \pi}\right]\right) \\
& +A_{\pi \rho}\left(\lambda_{u}^{(d)} \beta_{1}^{\pi \rho}+\sum_{p=u, c} \lambda_{p}^{(d)}\left[\beta_{4}^{p, \pi \rho}+\beta_{4, E W}^{p, \pi \rho}\right]\right)
\end{aligned}
$$




\begin{tabular}{|c|c|}
\hline Parameter & Relative Error \\
\hline$\gamma$ & $142.75 \%$ \\
\hline$X_{A}$ & $96.41 \%$ \\
\hline$X_{H}$ & $58.85 \%$ \\
\hline$\left|V_{u b} / V_{c b}\right|$ & $46.96 \%$ \\
\hline$m_{s}$ & $37.31 \%$ \\
\hline$\mu$ & $20.58 \%$ \\
\hline$a_{2}^{\rho}$ & $18.34 \%$ \\
\hline$\Lambda_{5}^{\mathrm{QCD}}$ & $13.16 \%$ \\
\hline$\lambda_{B}$ & $8.27 \%$ \\
\hline$A_{0}^{B \rightarrow \rho}$ & $7.06 \%$ \\
\hline$a_{2}^{\pi}$ & $6.26 \%$ \\
\hline$m_{b}$ & $5.22 \%$ \\
\hline$F_{+}^{B \rightarrow \pi}$ & $2.19 \%$ \\
\hline$\left|V_{u s}\right|$ & $1.38 \%$ \\
\hline$f_{\rho}$ & $0.93 \%$ \\
\hline
\end{tabular}

Table 4. Error budget for the observable $S_{\pi \rho}$ (Part I). Here $A_{0}^{B \rightarrow \rho}$ is the form factor for the transition $B \rightarrow \rho, a_{2}^{\rho}$ is the Gegenbauer moment for the leading twist LCDA for the $\rho$ meson.

with $\lambda_{u, c}^{(d)}$ given by eq. (2.2). In analogy with $S_{\pi \pi}$, there are also tree level amplitudes given by $\left\{\alpha_{2}^{\pi \rho}, \alpha_{2}^{\rho \pi}\right\}$, together with QCD and electroweak penguin contributions introduced through $\left\{\alpha_{4}^{\pi \rho}, \alpha_{4}^{\rho \pi}\right\}$ and $\left\{\tilde{\alpha}_{4}^{\pi \rho}, \tilde{\alpha}_{4}^{\rho \pi}\right\}$ respectively. Moreover, the coefficients $\left\{\beta_{1}^{p, \pi \rho}, \beta_{1}^{p, \rho \pi}\right\}$ correspond to current-current annihilation, $\left\{\beta_{3,4}^{p, \pi \rho}, \beta_{3,4}^{p, \rho \pi}\right\}$ to $\mathrm{QCD}$ penguin annihilation and $\left\{\beta_{4, E W}^{p, \pi \rho}, \beta_{4, E W}^{p, \rho \pi}\right\}$ to electroweak penguin annihilation. The TA can be obtained using eq. (2.20) and the information provided in appendix B. Our SM determination of the mixing induced CP asymmetry reads

$$
S_{\pi \rho}^{\mathrm{SM}}=-0.04 \pm 0.08
$$

which is compatible with the current experimental average [111]

$$
S_{\pi \rho}^{\operatorname{Exp}}=0.06 \pm 0.07
$$

The relative errors from each one of the inputs for $S_{\pi \rho}$ are presented in tables 4 and 5 , it can be seen that this observable is highly sensitive to the CKM input $\gamma$ leading to a relative uncertainty of $\mathcal{O}(100 \%)$. This is related to the fact that in the ratio $\lambda_{\rho \pi}$ given in eq. (4.29) we have:

$$
\operatorname{Re}\left(\frac{\mathcal{A}_{\rho^{+} \pi^{-}}}{A_{\pi^{+} \rho^{-}}}\right) \approx \operatorname{Im}\left(\frac{\mathcal{A}_{\rho^{+} \pi^{-}}}{A_{\pi^{+} \rho^{-}}}\right)
$$




\begin{tabular}{|c|c|}
\hline Parameter & Relative Error \\
\hline$f_{\pi}$ & $0.51 \%$ \\
\hline$f_{B}$ & $0.26 \%$ \\
\hline$f_{\rho}^{\perp}$ & $0.23 \%$ \\
\hline$\left|V_{c b}\right|$ & $0.06 \%$ \\
\hline$m_{c}$ & $0.02 \%$ \\
\hline Total & $194.57 \%$ \\
\hline
\end{tabular}

Table 5. Error budget for the observable $S_{\pi \rho}$ (Part II).

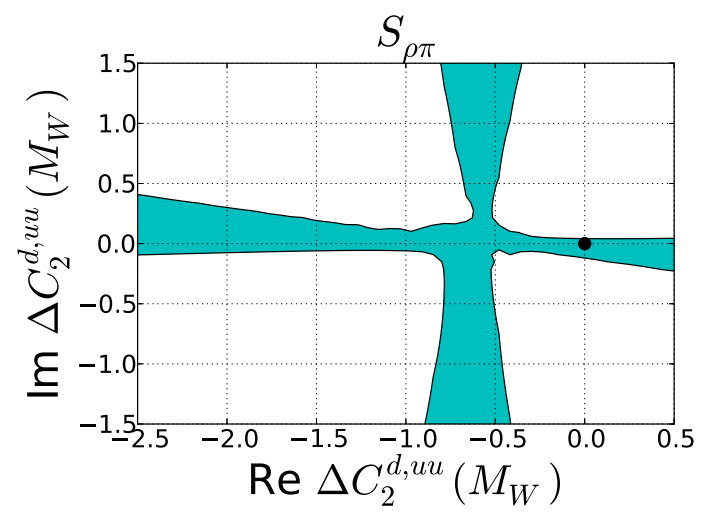

Figure 9. Potential regions for the NP contributions in $\Delta C_{2}^{d, u u}\left(M_{W}\right)$ allowed by the observable $S_{\rho \pi}$ at $90 \%$ C.L., the shift in the Wilson coefficient $\Delta C_{1}^{d, u u}\left(M_{W}\right)$ is only weakly constrained and therefore not shown. The black point corresponds to the SM value.

and

$$
\operatorname{Re}\left(\left[\frac{V_{t d} V_{t b}^{*}}{\left|V_{t d} V_{t b}^{*}\right|}\right]^{2}\right) \approx-\operatorname{Im}\left(\left[\frac{V_{t d} V_{t b}^{*}}{\left|V_{t d} V_{t b}^{*}\right|}\right]^{2}\right),
$$

which lead to a very strong cancellation on the resulting imaginary component. The allowed NP regions for $\Delta C_{2}^{d, u u}\left(M_{W}\right)$ are displayed in figure 9 . Here we can see how, in spite of having an uncertainty of $\mathcal{O}(100 \%)$, the observable $S_{\pi \rho}$ rules out large sections in the complex plane of $\Delta C_{2}^{d, u u}\left(M_{W}\right)$ and consequently deserves to be included in the analysis of $C_{2}^{d, u u}$. In contrast we find weak bounds for $\Delta C_{1}^{d, u u}\left(M_{W}\right)$ that are not strong enough to be taken into account. This is explained by the strong dependence of the amplitudes in eqs. (4.30) on $C_{2}^{d, u u}\left(M_{W}\right)$, which enters through $\alpha_{2}^{\pi \rho}$ and $\alpha_{2}^{\rho \pi}$ as shown in eq. (2.20).

\subsection{4 $R_{\rho \rho}$}

To obtain extra constraints on NP contributions to the tree level Wilson coefficients for the transition $b \rightarrow u \bar{u} d$ we include the ratio

$$
R_{\rho \rho}=\frac{\mathcal{B} r\left(B^{-} \rightarrow \rho_{L}^{-} \rho_{L}^{0}\right)}{\mathcal{B} r\left(\bar{B}_{d}^{0} \rightarrow \rho_{L}^{+} \rho_{L}^{-}\right)}=\left.\frac{\left|\mathcal{A}_{\rho^{-} \rho^{0}}\right|^{2}}{\left|\mathcal{A}_{\rho^{+}}\right|^{2}}\right|^{2}
$$


where $\mathcal{A}_{\rho^{-} \rho^{0}}$ and $\mathcal{A}_{\rho^{+} \rho-}$ are the amplitudes for the processes $B^{-} \rightarrow \rho_{L}^{-} \rho_{L}^{0}$ and $\bar{B}_{d}^{0} \rightarrow \rho_{L}^{+} \rho_{L}^{-}$ respectively. In terms of TAs they can be written as [97, 98]

$$
\begin{aligned}
\mathcal{A}_{\rho^{-}} \rho^{0}= & \frac{A_{\rho \rho}}{\sqrt{2}}\left[\lambda_{u}^{(d)}\left(\alpha_{1}^{\rho \rho}+\alpha_{2}^{\rho \rho}\right)+\frac{3}{2} \sum_{p=u, c} \lambda_{p}^{(d)}\left(\alpha_{7}^{p, \rho \rho}+\alpha_{9}^{p, \rho \rho}+\alpha_{10}^{p, \rho \rho}\right)\right], \\
\mathcal{A}_{\rho^{+} \rho^{-}}= & A_{\rho \rho}\left[\lambda_{u}^{(d)}\left(\alpha_{2}^{\rho \rho}+\beta_{2}^{\rho \rho}\right)+\sum_{p=u, c} \lambda_{p}^{(d)}\left(\alpha_{4}^{p, \rho \rho}+\alpha_{10}^{p, \rho \rho}\right.\right. \\
& \left.\left.+\beta_{3}^{p, \rho \rho}+2 \beta_{4}^{p, \rho \rho}-\frac{1}{2} \beta_{3, E W}^{p, \rho \rho}+\frac{1}{2} \beta_{4, E W}^{p, \rho \rho}\right)\right] .
\end{aligned}
$$

Here we expect a stronger dependence on $C_{1}$ compared to $C_{2}$. As indicated in eq. (4.36), in addition to the tree level contributions $\alpha_{1,2}^{\rho \rho}$, we can also identify QCD $\alpha_{4}^{\rho \rho}$ and electroweak penguins $\alpha_{7,9,10}^{\rho \rho}$. Moreover QCD penguin annihilation topologies enter through $\beta_{3,4}^{p, \rho \rho}$. On the other hand electroweak penguin annihilation is given by $\beta_{3,4, E W}^{p, \rho \rho}$. The expressions for the topological amplitudes obey the structure indicated in eq. (2.20) and can be calculated explicitly using the information provided in appendix B. Currently $\alpha_{1,2}^{\rho \rho}$ are available up to NNLO, we introduce these effects following the same procedure used for the determina-

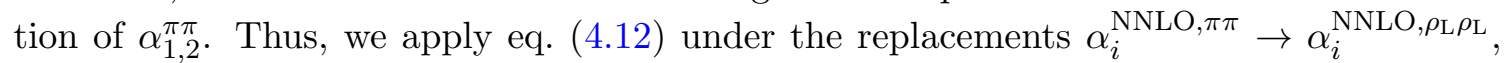
$\alpha_{i}^{\mathrm{NLO}, \pi \pi} \rightarrow \alpha_{i}^{\mathrm{NLO}, \rho_{\mathrm{L}} \rho_{\mathrm{L}}}$ and $\alpha_{i}^{\mathrm{NLO}, \pi \pi} \rightarrow \alpha_{0, i}^{\mathrm{NLO}, \rho_{\mathrm{L}} \rho_{\mathrm{L}}}$, with $i=1,2$. For the corresponding NNLO components we use [107]

$$
\begin{aligned}
& \alpha_{1}^{\mathrm{NNLO}, \rho_{L} \rho_{L}}=0.177_{-0.029}^{+0.025}-\left(0.097_{-0.029}^{+0.021}\right) i, \\
& \alpha_{2}^{\mathrm{NNLO}, \rho_{L} \rho_{L}}=1.017_{-0.011}^{+0.010}+\left(0.025_{-0.013}^{+0.019}\right) i .
\end{aligned}
$$

The uncertainty shown in eq. (4.37) has its origin in higher order perturbative corrections, we have taken this as the corresponding renormalization scale uncertainty when treating $\alpha_{1,2}^{\mathrm{NNLO}, \rho_{\mathrm{L}} \rho_{\mathrm{L}}}$ as nuisance parameters. Our SM determination for $R_{\rho \rho}$ is

$$
R_{\rho \rho}^{\mathrm{SM}}=(67.5 \pm 25.7) \cdot 10^{-2} .
$$

The experimental result for $R_{\rho \rho}$ is obtained by calculating the ratio of $\mathcal{B} r\left(B^{-} \rightarrow \rho_{L}^{-} \rho_{L}^{0}\right)$ and $\mathcal{B} r\left(\bar{B}_{d}^{0} \rightarrow \rho_{L}^{+} \rho_{L}^{-}\right)$weighted by the corresponding longitudinal polarization fractions $f_{L}^{-0}$ and $f_{L}^{+-}$. Using the numerical values available in the PDG [116] we obtain

$$
R_{\rho \rho}^{\text {Exp }}=(83.14 \pm 8.98) \cdot 10^{-2} .
$$

The partial contributions to the error budget are presented in table 6 and the constraints derived for $\Delta C_{1}^{d, u u}\left(M_{W}\right)$ in figure 10. We do not show the associated regions for $\Delta C_{2}^{d, u u}\left(M_{W}\right)$ because, for $R_{\rho \rho}$, the results are weaker than those derived from other observables in our study.

\subsection{Observables constraining $b \rightarrow c \bar{c} s$ transitions}

In this section we study bounds for $\Delta C_{1,2}^{s, c c}\left(M_{W}\right)$ stemming from $\mathcal{B} r\left(\bar{B} \rightarrow X_{s} \gamma\right)$, the mixing observable $\Delta \Gamma_{s}$, the CKM angle $\sin \left(2 \beta_{s}\right)$ and the lifetime ratio $\tau_{B_{s}} / \tau_{B_{d}}$. These observables give very constrained regions for $\Delta C_{1,2}^{s, c c}\left(M_{W}\right)$. 


\begin{tabular}{|c|c|}
\hline Parameter & Relative Error \\
\hline$X_{A}$ & $26.40 \%$ \\
\hline$X_{H}$ & $23.33 \%$ \\
\hline$\lambda_{B}$ & $12.32 \%$ \\
\hline$\mu$ & $6.78 \%$ \\
\hline$A_{0}^{B \rightarrow \rho}$ & $2.54 \%$ \\
\hline$a_{2}^{\rho}$ & $2.24 \%$ \\
\hline$f_{\rho}$ & $0.46 \%$ \\
\hline$\Lambda_{5}^{\mathrm{QCD}}$ & $0.45 \%$ \\
\hline$\gamma$ & $0.38 \%$ \\
\hline$m_{b}$ & $0.27 \%$ \\
\hline$f_{B}$ & $0.15 \%$ \\
\hline$f_{\rho}^{\perp}$ & $0.15 \%$ \\
\hline$m_{c}$ & $0.12 \%$ \\
\hline$f_{\rho}^{\perp}$ & $0.07 \%$ \\
\hline$\left|V_{u b} / V_{c b}\right|$ & $0.02 \%$ \\
\hline Total & $38.09 \%$ \\
\hline
\end{tabular}

Table 6. Error budget for the observable $R_{\rho \rho}$.

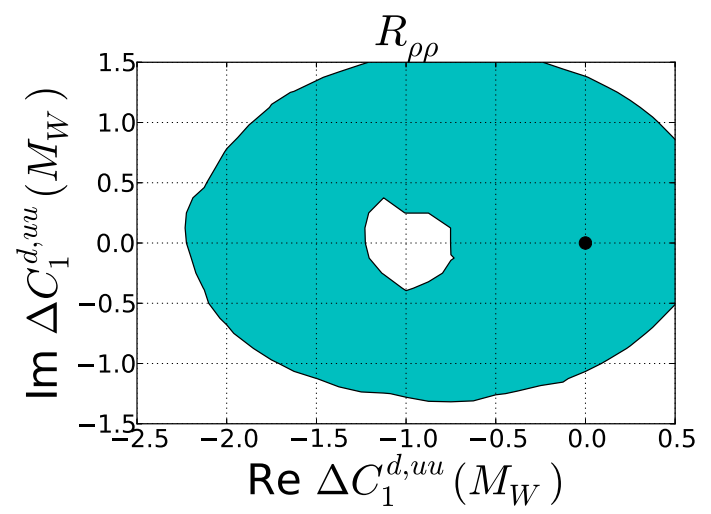

Figure 10. Potential regions for the NP contributions in $\Delta C_{1}^{d, u u}\left(M_{W}\right)$ allowed by the observable $R_{\rho \rho}$ at $90 \%$ C.L. . The bounds on $\Delta C_{2}^{d, u u}\left(M_{W}\right)$ are very weak and hence not shown. The black point corresponds to the SM value.

\subsection{1 $\bar{B} \rightarrow X_{s} \gamma$}

The process $\bar{B} \rightarrow X_{s} \gamma$ is of mayor interest for BSM studies for several reasons. To begin with, within the SM it is generated mainly at the loop level (its branching fraction actually receives contributions below $0.4 \%$ from the tree-level CKM-suppressed transitions $b \rightarrow u \bar{u} s \gamma$ when the energy of the photon is within the phenomenologically relevant range $\left.E_{\gamma} \geq 1.6 \mathrm{GeV}[123]\right)$. In the HQET, it corresponds to a flavour changing neutral current 
sensitive to new particles. Additionally, the experimental and theoretical precision achieved on its determination have an accuracy of the same order. Moreover, this transition is useful to constrain CKM elements involving the top quark.

The experimental world average for $\mathcal{B} r\left(\bar{B} \rightarrow X_{s} \gamma\right)$ up to date combines measurements from CLEO, Belle and BaBar leading to [111]

$$
\mathcal{B} r^{\operatorname{Exp}}\left(\bar{B} \rightarrow X_{s} \gamma\right)=(3.32 \pm 0.15) \cdot 10^{-4}
$$

On the theoretical side there has been a huge effort on the determination of this observable; the most precise results available are obtained at NNLO. Here we consider [124]

$$
\mathcal{B} r^{\mathrm{SM}}\left(\bar{B} \rightarrow X_{s} \gamma\right)=(3.36 \pm 0.22) \cdot 10^{-4}
$$

where the energy of the photon satisfies the cut

$$
E_{\gamma}>E_{0}=1.6 \mathrm{GeV}
$$

The calculation of the branching ratio for the process $\bar{B} \rightarrow X_{s} \gamma$ can be written as [125]

$$
\mathcal{B} r\left(\bar{B} \rightarrow X_{s} \gamma\right)_{E_{\gamma}>E_{0}}=\mathcal{B} r\left(\bar{B} \rightarrow X_{c} e \bar{\nu}\right)_{\exp }\left|\frac{V_{t s}^{*} V_{t b}}{V_{c b}}\right|^{2} \frac{6 \alpha_{\mathrm{em}}}{\pi C}\left[P\left(E_{0}\right)+N\left(E_{0}\right)\right]
$$

In eq. (4.43), $P\left(E_{0}\right)$ and $N\left(E_{0}\right)$ denote the perturbative and the non-perturbative contributions to the decay probability respectively. They depend on the lower cut for the energy of the photon in the Bremsstrahlung correction $E_{0}$ shown in eq. (4.42). Using the parameterisation given in ref. [126] we write $E_{0}=m_{b}^{1 S} / 2\left(1-\delta^{\prime}\right)$ and choose $\delta^{\prime}$ such that the lower bound in eq. (4.42) is saturated. The perturbative contribution $P\left(E_{0}\right)$ is given by $[125]$

$$
P\left(E_{0}\right)=\sum_{i, j=1}^{8} C_{i}^{\mathrm{eff}}\left(\mu_{b}\right) C_{j}^{\mathrm{eff} *}\left(\mu_{b}\right) K_{i j}\left(E_{0}, \mu_{b}\right)
$$

with $K_{i j}=\delta_{i 7} \delta_{j 7}+\mathcal{O}\left(\alpha_{s}\right)$. The effective Wilson coefficients $C_{i}^{\text {eff }}$ are expressed in terms of linear combinations of the coefficients for the operators $\hat{Q}_{i}^{s}(i=1, \ldots, 6), \hat{Q}_{7 \gamma}^{s}$ and $\hat{Q}_{8 g}^{s}$ introduced in section 2.1. For the denominator of eq. (4.43) we have [125]

$$
C=\left|\frac{V_{u b}}{V_{c b}}\right|^{2} \frac{\Gamma\left(\bar{B} \rightarrow X_{c} e \bar{\nu}\right)}{\Gamma\left(\bar{B} \rightarrow X_{u} e \bar{\nu}\right)}
$$

In order to account for the NNLO result in eq. (4.41) we write

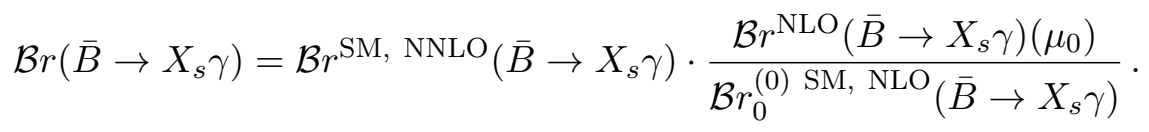




\begin{tabular}{|c|c|}
\hline Parameter & Relative error \\
\hline$N\left(E_{0}\right)$ & $5.00 \%$ \\
\hline$\mu$ & $3.00 \%$ \\
\hline $\mathcal{B} r\left(\bar{B} \rightarrow X_{c} e \bar{\nu}_{e}\right)$ & $2.68 \%$ \\
\hline$m_{c}\left(m_{c}\right)$ & $1.10 \%$ \\
\hline$m_{b}^{1 S}$ & $0.61 \%$ \\
\hline$\Lambda_{5}^{\mathrm{QCD}}$ & $0.26 \%$ \\
\hline$\gamma$ & $0.10 \%$ \\
\hline$\left|V_{u b} / V_{c b}\right|$ & $0.04 \%$ \\
\hline$\left|V_{u s}\right|$ & $0.01 \%$ \\
\hline Total & $6.55 \%$ \\
\hline
\end{tabular}

Table 7. Error budget for the observable $\mathcal{B} r\left(\bar{B} \rightarrow X_{s} \gamma\right)$. Here $N\left(E_{0}\right)$ determines the uncertainty arising from non-perturbative contributions.

Where

- $\mathcal{B r}^{\mathrm{NLO}}\left(\bar{B} \rightarrow X_{s} \gamma\right)$ is the branching ratio for the process $\bar{B} \rightarrow X_{s} \gamma$ calculated at NLO including NP effects from $\Delta C_{1,2}^{s, c c}\left(M_{W}\right)$. All inputs are allowed to float except the renormalisation scale, which is fixed at $\mu_{0}=m_{b}$. Our calculations are determined using the anomalous dimension matrices provided in [126]. NP contributions are introduced according to eq. (3.1). They propagate to the rest of the Wilson coefficients $C_{i}$ after applying the renormalisation group equations, described in section 2 of ref. [126].

- $\mathcal{B} r_{0}^{\mathrm{SM}, \mathrm{NLO}}\left(\bar{B} \rightarrow X_{s} \gamma\right)$ is the SM branching ratio for the process $\bar{B} \rightarrow X_{s} \gamma$ calculated at NLO and evaluated at the central values of all the input parameters and then kept constant during the $\chi^{2}$-fit.

- $\left.\mathcal{B}_{r} \mathrm{SM}, \mathrm{NNLO}_{(\bar{B}} \rightarrow X_{s} \gamma\right)$ is the SM branching ratio for the process $\bar{B} \rightarrow X_{s} \gamma$ calculated at NNLO and allowed to float within the uncertainty associated with the renormalisation scale. In the case of the theoretical result given in eq. (4.41) this corresponds to $3 \%$ of the central value [124]. ${ }^{5}$

The partial contributions to the final error are described in table 7. The allowed regions for $\Delta C_{1}^{s, c c}\left(M_{W}\right)$ and $\Delta C_{2}^{s, c c}\left(M_{W}\right)$ are shown in figure 11, where it can be seen how this observable imposes strong constraints on $\Delta C_{2}^{s, c c}\left(M_{W}\right)$. The bounds in figure 11 are consistent with those reported in [60] once a $68 \%$ C.L. is taken into account.

\footnotetext{
${ }^{5}$ In the NNLO determination in [124] two scales $\mu_{b}$ and $\mu_{c}$ are introduced. The $3 \%$ variation indicated in the error budget is derived from considering the variation $1.25 \mathrm{GeV} \leq \mu_{\mathrm{b}, \mathrm{c}} \leq 5 \mathrm{GeV}$ which accounts for about $2.4 \%$. However a more conservative value is taken due to the lack of certainty on extra contributions to the perturbation series involved, see more details in [124].
} 

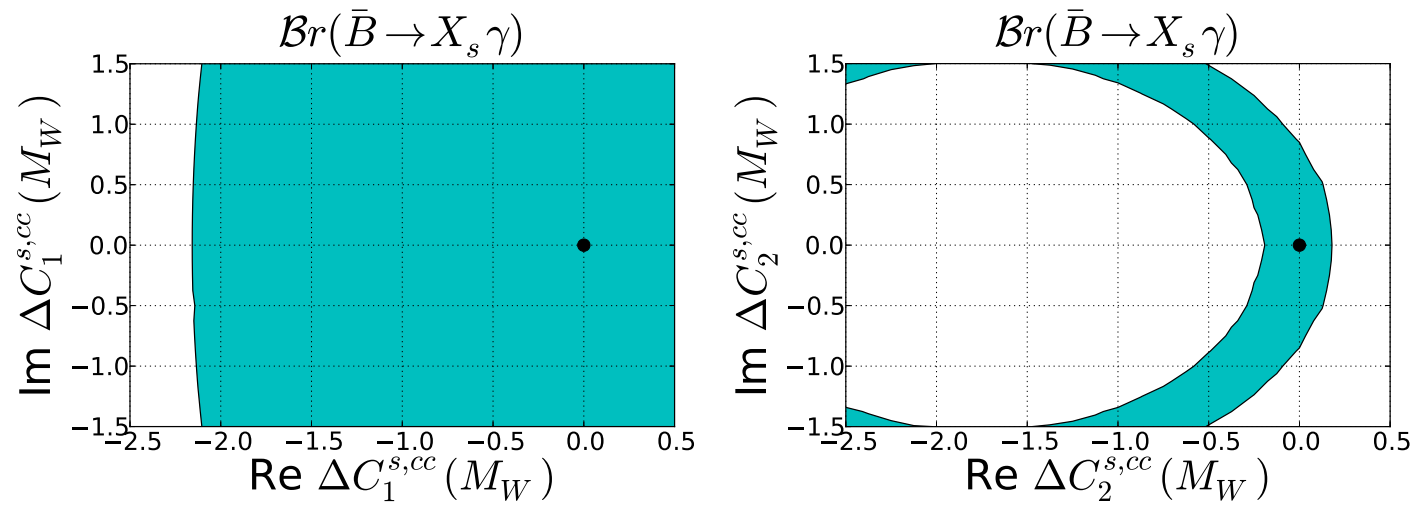

Figure 11. Potential regions for the NP contributions in $\Delta C_{1}^{s, c c}\left(M_{W}\right)$ and $\Delta C_{2}^{s, c c}\left(M_{W}\right)$ allowed by the observable $\mathcal{B} r\left(\bar{B} \rightarrow X_{s} \gamma\right)$ at $90 \%$ C.L. . The black point corresponds to the SM value.

\subsection{2 $\Delta \Gamma_{s}$ : bounds and SM update}

The decay rate differences $\Delta \Gamma_{q}$ and the semileptonic asymmetries $a_{s l}^{q}$ arising from neutral $B_{q}$ meson mixing are sensitive to the tree-level transitions $b \rightarrow u \bar{u} q, b \rightarrow u \bar{c} q, b \rightarrow c \bar{u} q$ and $b \rightarrow c \bar{c} q$ for $q=s, d$. We will, however, show below that for the decay rate difference of $B_{s}$-mesons our BSM study is completely dominated by the $b \rightarrow c \bar{c} s$ transition, yielding therefore strong constraints to $\Delta C_{1}^{s, c c}\left(M_{W}\right)$ and $\Delta C_{2}^{s, c c}\left(M_{W}\right)$.

The definitions of the observables $\Delta \Gamma_{q}$ and $a_{s l}^{q}$ in terms of $\Gamma_{12}^{q} / M_{12}^{q}$ were introduced in eqs. (2.16) and (2.17). Since, as explained in section 2.2, the elements $\Gamma_{12}^{q}$ are determined from the double insertion of $\hat{\mathcal{H}}_{\text {eff }}^{|\Delta B|=1}$ Hamiltonians, there are leading order contributions originating from the insertion of two current-current operators $\hat{Q}_{j}^{q, a b}$ for $a b=u u, u c, c c$ and $j=1,2$, see eq. (2.3). Additionally, there are also double insertions from a single currentcurrent $\hat{Q}_{1,2}^{q, a b}$ and a penguin operator $\hat{Q}_{3,4,5,6}^{q}$. In this section, we will only include NP effects to $\Gamma_{12}^{q}$, while we neglect tree level NP contributions to $M_{12}^{q}$ (these contribution are discussed in section 4.4.1 and they yield considerably weaker bounds for the observables $\Delta \Gamma_{q}$ and $\left.a_{s l}^{q}\right)$. To show the dominance of the $b \rightarrow c \bar{c} s$ contribution for $B_{s}$-mixing, we decompose $\Gamma_{12}^{q}$ into partial contributions $\Gamma_{12}^{q, a b}$, where the indices $a b=u u, u c, c c$ indicate which "up" type quarks are included inside the corresponding effective fermionic loops. Thus, the expression for $\Gamma_{12}^{q} / M_{12}^{q}$ becomes

$$
\begin{aligned}
\frac{\Gamma_{12}^{q}}{M_{12}^{q}} & =-\frac{\left(\lambda_{c}^{(q)}\right)^{2} \Gamma_{12}^{q, c c}+2 \lambda_{u}^{(q)} \lambda_{c}^{(q)} \Gamma_{12}^{q, u c}+\left(\lambda_{u}^{(q)}\right)^{2} \Gamma_{12}^{q, u u}}{M_{12}^{q}} \\
& =-\frac{\left(\lambda_{t}^{(q)}\right)^{2} \Gamma_{12}^{q, c c}+2 \lambda_{t}^{(q)} \lambda_{u}^{(q)}\left[\Gamma_{12}^{q, c c}-\Gamma_{12}^{q, u c}\right]+\left(\lambda_{u}^{(q)}\right)^{2}\left[\Gamma_{12}^{q, c c}-2 \Gamma_{12}^{q, u c}+\Gamma_{12}^{q, u u}\right]}{\left(\lambda_{t}^{(q)}\right)^{2} \tilde{M}_{12}^{q}} \\
& =-10^{-4}\left[c^{q}+a^{q} \frac{\lambda_{u}^{(q)}}{\lambda_{t}^{(q)}}+b^{q}\left(\frac{\lambda_{u}^{(q)}}{\lambda_{t}^{(q)}}\right)^{2}\right] .
\end{aligned}
$$

We have used here the unitarity of the CKM matrix: $\lambda_{u}^{(q)}+\lambda_{c}^{(q)}+\lambda_{t}^{(q)}=0$ and we have split off the CKM dependence from $M_{12}^{q}$ by introducing the quantity $\tilde{M}_{12}^{q}$. The GIM 
suppressed [127] terms $a$ and $b$ vanish in the limit $m_{c} \rightarrow m_{u}$ and the numerical values show a clear hierarchy

$$
c^{q} \approx-48, \quad a^{q} \approx 11, \quad b^{q} \approx 0.23 .
$$

For the ratio of CKM elements we obtain

$$
\begin{aligned}
\frac{\lambda_{u}^{(q)}}{\lambda_{t}^{(q)}} & \approx\left\{\begin{array}{c}
1.7 \cdot 10^{-2}-4.2 \cdot 10^{-1} i \text { for } q=d \\
-8.8 \cdot 10^{-3}+1.8 \cdot 10^{-2} i \text { for } q=s
\end{array}\right. \\
\left(\frac{\lambda_{u}^{(q)}}{\lambda_{t}^{(q)}}\right)^{2} & =\left\{\begin{array}{l}
-1.8 \cdot 10^{-1}-1.5 \cdot 10^{-2} i \text { for } q=d \\
-2.5 \cdot 10^{-4}-3.2 \cdot 10^{-4} i \text { for } q=s
\end{array}\right.
\end{aligned}
$$

Within the SM we find a very strong hierarchy of the three contributions in eq. (4.47). The by far largest term is given by $c^{q}$ and it is real. The second term proportional to $a^{q}$ is GIM and CKM suppressed - slightly for the case of $B_{d}$ mesons and more pronounced for $B_{s}$. Since $\lambda_{u}^{(q)} / \lambda_{t}^{(q)}$ is complex, this contribution gives rise to an imaginary part of $\Gamma_{12}^{q} / M_{12}^{q}$. Finally $b^{q}$ is even further GIM suppressed and again slightly/strongly CKM suppressed for $B_{d} / B_{s}$ mesons - this contribution has also both a real and an imaginary part. According to eqs. (2.16) the decay rate difference $\Delta \Gamma_{q}$, given by the real part of $\Gamma_{12}^{q} / M_{12}^{q}$, is dominated by the coefficient $c^{q}$ - stemming from $b \rightarrow c \bar{c} q$ transitions - and the coefficients $a^{q}$ and $b^{q}$ yield corrections of the order of 2 per mille. The semi-leptonic asymmetries are given by the imaginary part of $\Gamma_{12}^{q} / M_{12}^{q}$ (cf. eq. (2.17)), which in turn is dominated by the coefficient $a^{q}$, with $b^{q}$ giving sub-per mille corrections and no contributions from $c^{q}$.

Allowing new, complex contributions to $C_{1}$ and $C_{2}$ for individual quark level contributions we get the following effects:

1. The numerically leading coefficient $c^{q}$ can now also obtain an imaginary part.

2. The GIM cancellations in the coefficients $a^{q}$ and $b^{q}$ can be broken, if $b \rightarrow c \bar{c} q, b \rightarrow c \bar{u} q$, $b \rightarrow u \bar{c} q$ and $b \rightarrow u \bar{u} q$ are differently affected by NP. If there is a universal BSM contribution then the GIM cancellation will stay.

3. The CKM suppression will not be affected by our BSM modifications.

For the real part of $\Gamma_{12}^{s} / M_{12}^{s}$, we expect at most a correction of 2 per cent due to $a^{s}$ and $b^{s}$, even if the corresponding GIM suppression is completely lifted - thus $\Delta \Gamma_{s}$ is even in our BSM approach, completely dominated by $c^{s}$ and gives therefore only bounds on $b \rightarrow c \bar{c} s$. In the case of $B_{d}$ mesons, the corrections due to $a^{d}$ and $b^{d}$ could be as large as 40 per cent here all possible decay channels have to be taken into account - except we are considering universal BSM contributions to all decay channels. Since $\Delta \Gamma_{d}$ is not yet measured, we will revert our strategy and use the obtained bounds on the Wilson coefficients $C_{1}$ and $C_{2}$ to obtain potential enhancements or reductions of $\Delta \Gamma_{d}$ due to BSM effects in non-leptonic tree-level decays. Considering the imaginary part of $\Gamma_{12}^{s} / M_{12}^{s}$, we can get dramatically enhanced values for the semi-leptonic $\mathrm{CP}$ asymmetries, if $C_{1}$ or $C_{2}$ are complex, which will result in an imaginary part of the GIM-unsuppressed coefficient $c^{q}$. On the other hand new contributions to e.g. only $b \rightarrow c \bar{u} q$ or $b \rightarrow u \bar{c} q$ would have no effect on $c^{q}$, but they could lift 
the GIM suppression of the coefficient $a^{q}$ and thus lead to also large effects. Therefore the semileptonic $\mathrm{CP}$ asymmetries are not completely dominated by the $b \rightarrow c \bar{c} q$ transitions.

Next we explain in detail how to implement BSM contributions to $C_{1}$ and $C_{2}$ in the theoretical description of $\Gamma_{12}^{q}$. Each one of the functions $\Gamma_{12}^{q, a b}$ in eq. (4.47) are given by [87]

$$
\Gamma_{12}^{q, a b}=\frac{G_{F}^{2} m_{b}^{2}}{24 \pi M_{B_{q}}}\left[\left(G^{q, a b}+\frac{1}{2} \alpha_{2} G_{S}^{q, a b}\right)\left\langle B_{q}\left|\hat{Q}_{1}\right| \bar{B}_{q}\right\rangle+\alpha_{1} G_{S}^{q, a b}\left\langle B_{q}\left|\hat{Q}_{3}\right| \bar{B}_{q}\right\rangle\right]+\tilde{\Gamma}_{12,1 / m_{b}}^{q, a b} .
$$

The coefficients $\alpha_{1}$ and $\alpha_{2}$ in eq. (4.51) include NLO corrections and are written in the $\overline{\mathrm{MS}}$ scheme as

$$
\alpha_{1}=1+\frac{\alpha_{s}(\mu)}{4 \pi} C_{F}\left(12 \ln \frac{\mu}{m_{b}}+6\right), \quad \alpha_{2}=1+\frac{\alpha_{s}(\mu)}{4 \pi} C_{F}\left(12 \ln \frac{\mu}{m_{b}}+\frac{13}{2}\right) .
$$

Furthermore, the expressions for $G^{q, a b}$ and $G_{S}^{q, a b}$ in eq. (4.51) are decomposed as

$$
G^{q, a b}=F^{q, a b}+P^{q, a b}, \quad G_{S}^{q, a b}=-F_{S}^{q, a b}-P_{S}^{q, a b},
$$

with $F^{q, a b}$ and $F_{S}^{q, a b}$ encoding the perturbative contributions resulting from the double insertion of current-current operators. Finally, $P^{q, a b}$ and $P_{S}^{q, a b}$ contain the perturbative effects from the combined insertion of a current-current and a penguin operators. In terms of the tree-level Wilson coefficients $C_{1}^{q, a b}$ and $C_{2}^{q, a b}$, the equations for $F^{q, a b}$ and $F_{S}^{q, a b}$ have the following generic structure

$$
F^{q, a b}=F_{11}^{q, a b}\left[C_{1}^{q, a b}(\mu)\right]^{2}+F_{12}^{q, a b} C_{1}^{q, a b}(\mu) C_{2}^{q, a b}(\mu)+F_{22}^{q, a b}\left[C_{2}^{q, a b}(\mu)\right]^{2},
$$

where the individual factors $F_{11,12,22}^{q, a b}$ are available in the literature up to NLO

$$
F_{i j}^{q, a b}=F_{i j}^{q,(0)}+\frac{\alpha_{s}(\mu)}{4 \pi} F_{i j}^{q,(1)} .
$$

To account for NP effects, the Wilson coefficients inside eq. (4.54) should be determined using eq. (3.1) and applying the renormalization group equations introduced in section 2 . Notice that eq. (4.54) is sensitive to the different transitions $b \rightarrow c \bar{c} q, b \rightarrow u \bar{c} q, b \rightarrow$ $c \bar{u} q$ and $b \rightarrow u \bar{u} q$. To be consistent with the inclusion of NP effects $\Delta C_{1}^{q, a b}\left(M_{W}\right)$ and $\Delta C_{2}^{q, a b}\left(M_{W}\right)$ at LO only, we omit all the terms involving products between $\alpha_{s}(\mu)$ and the NP factors $\Delta C_{1,2}^{q, a b}\left(M_{W}\right)$ inside eq. (4.54). The penguin functions $P^{q, a b}$ and $P_{S}^{q, a b}$ also contain LO contributions from $C_{1,2}^{q, a b}$. For the purposes of illustration we will show the explicit expressions for the functions $P^{s, c c}$ and $P_{S}^{s, c c}$ corresponding to the $B_{s}^{0}-\bar{B}_{s}^{0}$ system. At NLO we have [67]

$$
\begin{aligned}
P^{s, c c}= & \sqrt{1-4 \bar{z}}\left[(1-\bar{z}) K_{1}^{\prime c c}(\mu)+\frac{1}{2}(1-4 \bar{z}) K_{2}^{\prime c c}(\mu)+3 \bar{z} K_{3}^{\prime c c}(\mu)\right] \\
& +\frac{\alpha_{s}(\mu)}{4 \pi} F_{p}^{c c}(\bar{z})\left[C_{2}^{s, c c}(\mu)\right]^{2}, \\
P_{S}^{s, c c}= & \sqrt{1-4 \bar{z}}[1+2 \bar{z}]\left[K_{1}^{\prime c c}(\mu)-K_{2}^{\prime c c}(\mu)\right]-\frac{\alpha_{s}(\mu)}{4 \pi} 8 F_{p}(\bar{z})\left[C_{2}^{s, c c}(\mu)\right]^{2} .
\end{aligned}
$$


Where the following definition for the ratio of the masses of the bottom and charm quarks, evaluated in the $\overline{\mathrm{MS}}$ scheme [87], has been used

$$
\bar{z}=\left[\bar{m}_{c}\left(\bar{m}_{b}\right) / \bar{m}_{b}\left(\bar{m}_{b}\right)\right]^{2} .
$$

The functions $K_{1,2,3}^{\prime c c}$ inside eq. (4.56) are given by

$$
\begin{aligned}
& K_{1}^{\prime c c}(\mu)=2\left[3 C_{1}^{s, c c}(\mu) C_{3}^{s}(\mu)+C_{1}^{s, c c}(\mu) C_{4}^{s}(\mu)+C_{2}^{s, c c}(\mu) C_{3}^{s}(\mu)\right] \\
& K_{2}^{\prime c c}(\mu)=2 C_{2}^{s, c c}(\mu) C_{4}^{s}(\mu), \\
& K_{3}^{\prime c c}(\mu)=2\left[3 C_{1}^{s, c c}(\mu) C_{5}^{s}(\mu)+C_{1}^{s, c c}(\mu) C_{6}(\mu)+C_{2}^{s, c c}(\mu) C_{5}^{s}(\mu)+C_{2}^{s, c c}(\mu) C_{6}^{s}(\mu)\right],
\end{aligned}
$$

and the expression for the NLO correction function $F_{p}^{c c}(z)$ is

$$
\begin{aligned}
F_{p}^{c c}(z)= & -\frac{1}{9} \sqrt{1-4 \bar{z}}(1+2 \bar{z})\left[2 \ln \frac{\mu}{m_{b}}+\frac{2}{3}+4 \bar{z}-\ln \bar{z}\right. \\
& \left.+\sqrt{1-4 \bar{z}}(1+2 \bar{z}) \ln \frac{1-\sqrt{1-4 \bar{z}}}{1+\sqrt{1+4 \bar{z}}}+\frac{3 C_{8 g}^{s}(\mu)}{C_{2}^{s, c c}(\mu)}\right]
\end{aligned}
$$

The Wilson coefficients inside eqs. (4.58) should be calculated by introducing NP deviations at the scale $\mu=M_{W}$ and then running down their corresponding values to the scale $\mu \sim m_{b}$ through the renormalization group equations, for details see the discussion in section 2 . In appendix A, we provide details on the numerical inputs used. Since there was tremendous progress $[64,65]$ in the theoretical precision of the mixing observables we will present in this work numerical updates of all mixing observables: $\Delta \Gamma_{q}$ below, $\Delta M_{q}$ in section 4.4.1 and the semi-leptonic CP asymmetries $a_{s l}^{q}$ and $\phi_{q}$ in section 4.5. For our numerical analysis we use results for $\Gamma_{12,3}^{q,(0)}, \Gamma_{12,3}^{q,(1)}$ and $\Gamma_{12,4}^{q,(0)}$, from $[67,80,83-87]$ and for the hadronic matrix elements the averages presented in [65] based on [82, 90, 91] and [92-95], as well as the dimension seven matrix elements from [96]. The new SM determinations for $\Delta \Gamma_{s}$ and $\Delta \Gamma_{d}$ are

$$
\begin{aligned}
& \Delta \Gamma_{s}^{\mathrm{SM}}=(9.1 \pm 1.3) \cdot 10^{-2} \mathrm{ps}^{-1}, \\
& \Delta \Gamma_{d}^{\mathrm{SM}}=(2.6 \pm 0.4) \cdot 10^{-3} \mathrm{ps}^{-1} .
\end{aligned}
$$

The error budgets of the mixing observables $\Delta \Gamma_{s}$ and $\Delta \Gamma_{d}$ are presented in tables 8 and 9 respectively. Compared to the SM estimates for $\Delta \Gamma_{s}$ stemming from 2006 [87], 2011 [128] and 2015 [31] we find a huge improvement in the SM precision. Moreover, the value of $\Delta \Gamma_{s}$ in eq. (4.60) is in good agreement with the corresponding result of $\Delta \Gamma_{s}=(9.2 \pm 1.4) \cdot 10^{-2} \mathrm{ps}^{-1}$ obtained in [96].

In addition, the current SM predictions are based for the first time on a nonperturbative determination [96] of the leading uncertainty due to dimension seven operators. All previous predictions had to rely on vacuum insertion approximation for the corresponding matrix elements. To further reduce the theory uncertainties, improvements in the lattice determination would be very welcome or a corresponding sum rule calculation. The next important uncertainty stems from the renormalisation scale dependence, 


\begin{tabular}{|c|c|c|c|c|}
\hline$\Delta \Gamma_{s}^{\mathrm{SM}}$ & this work & ABL 2015 & LN 2011 & LN 2006 \\
\hline Central Value & $0.091 \mathrm{ps}^{-1}$ & $0.088 \mathrm{ps}^{-1}$ & $0.087 \mathrm{ps}^{-1}$ & $0.096 \mathrm{ps}^{-1}$ \\
\hline$B_{\widetilde{R}_{2}}^{s}$ & $10.9 \%$ & $14.8 \%$ & $17.2 \%$ & $15.7 \%$ \\
\hline$\mu$ & $6.6 \%$ & $8.4 \%$ & $7.8 \%$ & $13.7 \%$ \\
\hline$V_{c b}$ & $3.4 \%$ & $4.9 \%$ & $3.4 \%$ & $4.9 \%$ \\
\hline$B_{R_{0}}^{s}$ & $3.2 \%$ & $2.1 \%$ & $3.4 \%$ & $3.0 \%$ \\
\hline$f_{B_{s}} \sqrt{B_{1}^{s}}$ & $3.1 \%$ & $13.9 \%$ & $13.5 \%$ & $34.0 \%$ \\
\hline$B_{3}^{s}$ & $2.2 \%$ & $2.1 \%$ & $4.8 \%$ & $3.1 \%$ \\
\hline $\bar{z}$ & $0.9 \%$ & $1.1 \%$ & $1.5 \%$ & $1.9 \%$ \\
\hline$m_{b}$ & $0.9 \%$ & $0.8 \%$ & $0.1 \%$ & $1.0 \%$ \\
\hline$B_{R_{3}}^{s}$ & $0.5 \%$ & $0.2 \%$ & $0.2 \%$ & - \\
\hline$B_{\tilde{R}_{3}}^{s}$ & - & $0.6 \%$ & $0.5 \%$ & - \\
\hline$m_{s}$ & $0.3 \%$ & $0.1 \%$ & $1.0 \%$ & $1.0 \%$ \\
\hline$B_{\tilde{R}_{1}}^{s}$ & $0.2 \%$ & $0.7 \%$ & $1.9 \%$ & - \\
\hline$\Lambda_{5}^{\mathrm{QCD}}$ & $0.1 \%$ & $0.1 \%$ & $0.4 \%$ & $0.1 \%$ \\
\hline$\gamma$ & $0.1 \%$ & $0.1 \%$ & $0.3 \%$ & $1.0 \%$ \\
\hline$B_{R_{1}}^{s}$ & $0.1 \%$ & $0.5 \%$ & $0.8 \%$ & - \\
\hline$\left|V_{u b} / V_{c b}\right|$ & $0.1 \%$ & $0.1 \%$ & $0.2 \%$ & $0.5 \%$ \\
\hline $\bar{m}_{t}\left(\bar{m}_{t}\right)$ & $0.0 \%$ & $0.0 \%$ & $0.0 \%$ & $0.0 \%$ \\
\hline Total $\left.^{\prime}\right)$ & $14.1 \%$ & $22.8 \%$ & $24.5 \%$ & $40.5 \%$ \\
\hline & & & & \\
\hline
\end{tabular}

Table 8. List of the individual contributions to the theoretical error of the decay rate difference $\Delta \Gamma_{s}$ within the Standard Model and comparison with the values obtained in 2015 [31], in 2011 [128] and in 2006 [87]. We have used equations of motion in the current analysis to get rid of the operator $\tilde{R}_{3}$.

to reduce this a NNLO calculation is necessary. First steps in that direction have been done in [129]. In the ratio $\Delta \Gamma_{q} / \Delta M_{q}$ uncertainties due to the matrix elements of dimension six are cancelling - so for a long time this ratio was considerably better known than the individual value of $\Delta \Gamma_{s}$. Due to the huge progress in determining precise values for these non-perturbative parameter, this advantage is now considerably less pronounced, see table 10. For the corresponding experimental values we use the HFLAV averages [111]

$$
\begin{aligned}
& \Delta \Gamma_{s}^{\operatorname{Exp}}=(8.8 \pm 0.6) \cdot 10^{-2} \mathrm{ps}^{-1}, \\
& \Delta \Gamma_{d}^{\operatorname{Exp}}=(-1.3 \pm 6.6) \cdot 10^{-3} \mathrm{ps}^{-1},
\end{aligned}
$$

where $\Delta \Gamma_{d}^{E x p}$ was obtained using [111]

$$
\left(\Delta \Gamma_{d} / \Gamma_{d}\right)^{\operatorname{Exp}}=-0.002 \pm 0.010, \quad \tau_{B_{d}^{0}}^{\operatorname{Exp}}=(1.520 \pm 0.004) \mathrm{ps} .
$$

The resulting regions for $\Delta C_{1}^{s, c c}\left(M_{W}\right)$ and $\Delta C_{2}^{s, c c}\left(M_{W}\right)$ allowed by $\Delta \Gamma_{s}$ are presented in figure 12 . 


\begin{tabular}{|c|c|c|}
\hline$\Delta \Gamma_{d}^{\mathrm{SM}}$ & This work & ABL 2015 \\
\hline Central Value & $2.61 \cdot 10^{-3} \mathrm{ps}^{-1}$ & $2.61 \cdot 10^{-3} \mathrm{ps}^{-1}$ \\
\hline$B_{\widetilde{R}_{2}}^{d}$ & $11.1 \%$ & $14.4 \%$ \\
\hline$f_{B_{d}} \sqrt{B_{1}^{d}}$ & $3.6 \%$ & $13.7 \%$ \\
\hline$\mu$ & $6.7 \%$ & $7.9 \%$ \\
\hline$V_{c b}$ & $3.4 \%$ & $4.9 \%$ \\
\hline$B_{3}^{d}$ & $2.4 \%$ & $4.0 \%$ \\
\hline$B_{R_{0}}^{d}$ & $3.3 \%$ & $2.5 \%$ \\
\hline $\bar{z}$ & $0.9 \%$ & $1.1 \%$ \\
\hline$m_{b}$ & $0.9 \%$ & $0.8 \%$ \\
\hline$\tilde{B}_{R_{3}}^{d}$ & - & $0.5 \%$ \\
\hline$B_{R_{3}}^{d}$ & $0.5 \%$ & $0.2 \%$ \\
\hline$\gamma$ & $2.2 \%$ & $2.5 \%$ \\
\hline$\Lambda_{5}^{\mathrm{QCD}}$ & $0.1 \%$ & $0.1 \%$ \\
\hline$\left|V_{u b} / V_{c b}\right|$ & $0.0 \%$ & $0.1 \%$ \\
\hline $\bar{m}_{t}\left(\bar{m}_{t}\right)$ & $0.0 \%$ & $0.0 \%$ \\
\hline Total & $14.7 \%$ & $22.7 \%$ \\
\hline
\end{tabular}

Table 9. List of the individual contributions to the theoretical error of the mixing quantity $\Delta \Gamma_{d}$ and comparison with the values obtained in 2015 [31]. We have used equations of motion in the current analysis to get rid of the operator $\tilde{R}_{3}$.
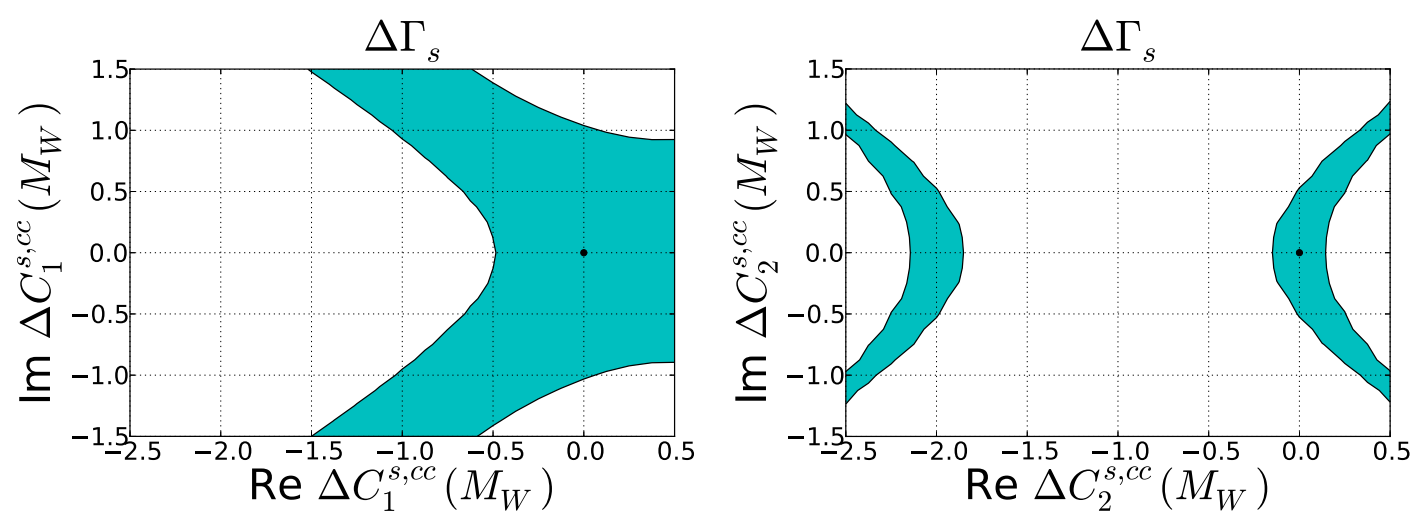

Figure 12. Potential regions for the NP contributions in $\Delta C_{1}^{s, c c}\left(M_{W}\right)$ and $\Delta C_{2}^{s, c c}\left(M_{W}\right)$ allowed by the observable $\Delta \Gamma_{s}$ at $90 \%$ C.L. . The black point corresponds to the SM value. 


\begin{tabular}{|c|c|c|c|c|}
\hline$\Delta \Gamma_{s}^{\mathrm{SM}} / \Delta M_{s}^{\mathrm{SM}}$ & this work & ABL 2015 & $\mathrm{LN} 2011$ & $\mathrm{LN} 2006$ \\
\hline Central Value & $48.2 \cdot 10^{-4}$ & $48.1 \cdot 10^{-4}$ & $50.4 \cdot 10^{-4}$ & $49.7 \cdot 10^{-4}$ \\
\hline$B_{R_{2}}^{s}$ & $10.9 \%$ & $14.8 \%$ & $17.2 \%$ & $15.7 \%$ \\
\hline$\mu$ & $6.6 \%$ & $8.4 \%$ & $7.8 \%$ & $9.1 \%$ \\
\hline$B_{R_{0}}^{s}$ & $3.2 \%$ & $2.1 \%$ & $3.4 \%$ & $3.0 \%$ \\
\hline$B_{3}^{s}$ & $2.2 \%$ & $2.1 \%$ & $4.8 \%$ & $3.1 \%$ \\
\hline $\bar{z}$ & $0.9 \%$ & $1.1 \%$ & $1.5 \%$ & $1.9 \%$ \\
\hline$m_{b}$ & $0.9 \%$ & $0.8 \%$ & $1.4 \%$ & $1.0 \%$ \\
\hline$B_{R_{3}}^{s}$ & $0.5 \%$ & $0.2 \%$ & $0.2 \%$ & - \\
\hline$B_{\tilde{R}_{3}}^{s}$ & - & $0.6 \%$ & $0.5 \%$ & - \\
\hline $\bar{m}_{t}\left(\bar{m}_{t}\right)$ & $0.3 \%$ & $0.7 \%$ & $1.1 \%$ & $1.8 \%$ \\
\hline$m_{s}$ & $0.3 \%$ & $0.1 \%$ & $1.0 \%$ & $0.1 \%$ \\
\hline$\Lambda_{5}^{\mathrm{QCD}}$ & $0.2 \%$ & $0.2 \%$ & $0.8 \%$ & $0.1 \%$ \\
\hline$B_{\tilde{R}_{1}}^{s}$ & $0.2 \%$ & $0.7 \%$ & $1.9 \%$ & - \\
\hline$B_{R_{1}}^{s}$ & $0.1 \%$ & $0.5 \%$ & $0.8 \%$ & - \\
\hline$\gamma$ & $<0.1 \%$ & $0.0 \%$ & $0.0 \%$ & $0.1 \%$ \\
\hline $\mid V_{u b} / V_{c b}$ & $<0.1 \%$ & $0.0 \%$ & $0.0 \%$ & $0.1 \%$ \\
\hline$V_{c b}$ & $<0.1 \%$ & $0.0 \%$ & $0.0 \%$ & $0.0 \%$ \\
\hline Total & $13.4 \%$ & $17.3 \%$ & $20.1 \%$ & $18.9 \%$ \\
\hline
\end{tabular}

Table 10. List of the individual contributions to the theoretical error of the ratio $\Delta \Gamma_{s} / \Delta M_{s}$ within the Standard Model and comparison with the values obtained in 2015 [31], in 2011 [128] and in 2006 [87]. We have used equations of motion in the current analysis to get rid of the operator $\tilde{R}_{3}$.

\subsection{3 $\quad S_{J / \psi \phi}$}

The mixing induced CP asymmetry for the decay $\bar{B}_{s} \rightarrow J / \psi \phi$, given as

$$
S_{J / \psi \phi}=\frac{2 \operatorname{Im}\left(\lambda_{J / \psi \phi}^{s}\right)}{1+\left|\lambda_{J / \psi \phi}^{s}\right|^{2}}=\sin \left(2 \beta_{s}\right),
$$

can be used to constrain $\Delta C_{1}^{s, c c}\left(M_{W}\right)$. In eq. (4.64), $\lambda_{J / \psi \phi}^{s}$ is determined according to eq. (4.20) considering the amplitudes $\overline{\mathcal{A}}_{J / \psi \phi}$ and $\mathcal{A}_{J / \psi \phi}$ for the decays $\bar{B}_{s}^{0} \rightarrow J / \psi \phi$ and $B_{s}^{0} \rightarrow J / \psi \phi$ respectively. The required theoretical expressions have been calculated explicitly within the QCDF formalism in [130]. The equation for the decay amplitude obeys the structure

$$
\mathcal{A}_{J / \psi \phi}^{h} \propto \alpha_{1}^{J / \psi \phi, h}+\alpha_{3}^{J / \psi \phi, h}+\alpha_{5}^{J / \psi \phi, h}+\alpha_{7}^{J / \psi \phi, h}+\alpha_{9}^{J / \psi \phi, h},
$$

where the proportionality constant has been omitted since it cancels in the ratio $\lambda_{J / \psi \phi}^{s}$. The amplitudes $\alpha_{i}^{J / \psi \phi}$ appearing in eq. (4.65) obey the structure given in eq. (2.20). The 


\begin{tabular}{|c|c|}
\hline Parameter & Relative error \\
\hline$\left|V_{u b} / V_{c b}\right|$ & $2.44 \%$ \\
\hline$\gamma$ & $1.39 \%$ \\
\hline$\left|V_{u s}\right|$ & $0.07 \%$ \\
\hline Total & $2.81 \%$ \\
\hline
\end{tabular}

Table 11. Error budget for the observable $\sin \left(2 \beta_{s}\right)$.

required expressions for the vertices and hard-scattering functions can be found in the appendix. The index $h=0, \pm$ indicated in eq. (4.65) makes reference to helicity of the particles in the final state. During our analysis we average over the different helicity contributions. Therefore we take

$$
S_{J / \psi \phi}=\frac{S_{J / \psi \phi}^{0}+S_{J / \psi \phi}^{+}+S_{J / \psi \phi}^{-}}{3},
$$

where each one of the asymmetries $S_{J / \psi \phi}^{h}$, are determined individually considering the corresponding amplitude $\mathcal{A}_{J / \psi \phi}^{h}$ for $h=0, \pm$.

Neglecting penguin contributions our theoretical evaluation leads to

$$
\sin \left(2 \beta_{s}^{\mathrm{SM}}\right)=0.037 \pm 0.001,
$$

which numerically coincides with $2 \beta_{s}^{\mathrm{SM}}$ within the precision under consideration. The error budget is shown in table 11. On the experimental side we use the average [111]

$$
2 \beta_{s}^{\text {Exp }}=0.021 \pm 0.031 \text {. }
$$

The effect of $S_{J / \psi \phi}$ on the allowed values for $\Delta C_{1}^{s, c c}\left(M_{W}\right)$ is not as strong as the results derived from other observables. However we included it in our analysis for completeness. For this reason we do not show the individual constraints from $S_{J / \psi \phi}$ and present only its effect in the global $\chi^{2}$-fit described in section 5.4.

\subsection{4 $\tau_{B_{s}} / \tau_{B_{d}}$}

The lifetime ratio $\tau_{B_{s}} / \tau_{B_{d}}$ gives us sensitivity to $\Delta C_{1}^{s, c c}\left(M_{W}\right)$ and $\Delta C_{2}^{s, c c}\left(M_{W}\right)$ via the weak exchange diagram contributing to the $B_{s}$-lifetime as CKM leading part. We assumed here that no new effects are arising in the $B_{d}$-lifetime, where the CKM leading part is given by a $b \rightarrow c \bar{u} d$ transition. Allowing new effects in both $b \rightarrow c \bar{c} s$ and $b \rightarrow c \bar{u} d$ the individually large effects will hugely cancel. We also neglect the currently unknown contribution of the Darwin term. ${ }^{6}$

Using the results presented in [58] we write

$$
\frac{\tau_{B_{s}}}{\tau_{B_{d}}}=\left(\frac{\tau_{B_{s}}}{\tau_{B_{d}}}\right)^{\mathrm{SM}}+\left(\frac{\tau_{B_{s}}}{\tau_{B_{d}}}\right)^{\mathrm{NP}}
$$

\footnotetext{
${ }^{6}$ Recently the Wilson coefficient of the Darwin operator was found to be large [131, 132] for B mesons. Due to the currently unknown size of the matrix element of this operator in between $B_{s}$ states, the numerical effect of these new contributions on the lifetime ratio $\tau_{B_{s}} / \tau_{B_{d}}$ - being proportional to $\left\langle B_{s}\left|\rho_{D}^{3}\right| B_{s}\right\rangle-$ $\left\langle B_{d}\left|\rho_{D}^{3}\right| B_{d}\right\rangle$ - cannot yet be estimated.
} 
for the SM value we take [82]

$$
\left(\frac{\tau_{B_{s}}}{\tau_{B_{d}}}\right)^{\mathrm{SM}}=1.0006 \pm 0.0020
$$

The experimental result for the ratio is [111]

$$
\left(\frac{\tau_{B_{s}}}{\tau_{B_{d}}}\right)^{\operatorname{Exp}}=0.994 \pm 0.004
$$

To estimate the NP contribution $\left(\tau_{B_{s}} / \tau_{B_{d}}\right)^{\mathrm{NP}}$ we consider the following function [58]

$$
\begin{aligned}
F_{\tau_{B_{s}} / \tau_{B_{d}}}\left(C_{1}, C_{2}\right)= & G_{F}^{2}\left|V_{c b} V_{c s}\right|^{2} m_{b}^{2} M_{B_{s}} f_{B_{s}}^{2} \tau_{B_{s}} \frac{\sqrt{1-4 x_{c}^{2}}}{144 \pi}\left\{( 1 - x _ { c } ^ { 2 } ) \left[4\left|C^{\prime}\right|^{2} B_{1}\right.\right. \\
& \left.\left.+24\left|C_{2}\right|^{2} \epsilon_{1}\right]-\frac{M_{B_{s}}^{2}\left(1+2 x_{c}^{2}\right)}{\left(m_{b}+m_{s}\right)^{2}}\left[4\left|C^{\prime}\right|^{2} B_{2}+24\left|C_{2}\right|^{2} \epsilon_{2}\right]\right\},
\end{aligned}
$$

where $x_{c}=m_{c} / m_{b}$ and $C^{\prime}$ denotes the following combination of tree-level Wilson coefficients

$$
C^{\prime} \equiv 3 C_{1}+C_{2}
$$

The non-perturbative matrix elements of the arising four-quark $\Delta B=0$ operators are parameterised in terms of the decay constant $f_{B_{s}}$ and the bag parameter $B_{1}, B_{2}, \epsilon_{1}$ and $\epsilon_{2}$, which we take from the recent evaluation in [82]. The numerical values used are listed in appendix A. The NP contribution to the lifetime ratio can be written as

$$
\begin{aligned}
\left(\frac{\tau_{B_{s}}}{\tau_{B_{d}}}\right)^{\mathrm{NP}}= & F_{\tau_{B_{s}} / \tau_{B_{d}}}\left(C_{1}^{s, c c}(\mu), C_{2}^{s, c c}(\mu)\right) \\
& -\left.F_{\tau_{B_{s}} / \tau_{B_{d}}}\left(C_{1}^{s, c c}(\mu), C_{2}^{s, c c}(\mu)\right)\right|_{\mathrm{SM}},
\end{aligned}
$$

where in the second term in eq. (4.74) we have dropped the NP contributions $\Delta C_{1}^{s, c c}(\mu)$ and $\Delta C_{2}^{s, c c}(\mu)$. Our bounds for $\Delta C_{1}^{s, c c}\left(M_{W}\right)$ are shown in figure 13, the corresponding results for $\Delta C_{2}^{s, c c}\left(M_{W}\right)$ turn out to be weak and therefore we do not display them. We would like to highlight the consistency between our regions and those presented in [60] which were calculated at the $68 \%$ C.L. .

\subsection{Observables constraining $b \rightarrow c \bar{c} d$ transitions}

We devote this section to the derivation of bounds on $\Delta C_{1}^{d, c c}\left(M_{W}\right)$ and $\Delta C_{2}^{d, c c}\left(M_{W}\right)$ from $\sin \left(2 \beta_{d}\right)$ and $B \rightarrow X_{d} \gamma$. In our final analysis we also included contributions from $a_{s l}^{d}$ which will be described in more detail in section 4.5.

\subsection{1 $\sin \left(2 \beta_{d}\right)$ and SM update of $\Delta M_{q}$}

In our BSM framework mixing induced CP asymmetries can be modified by changes in the tree-level decay or by changes to the neutral $B$-meson mixing. The first effect was 


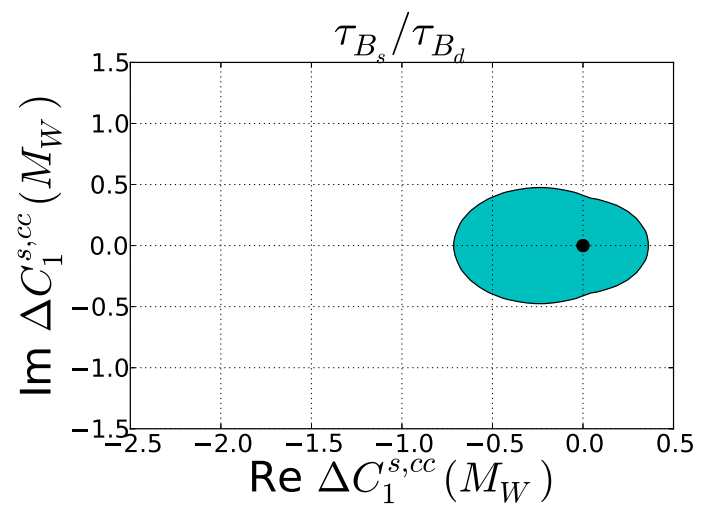

Figure 13. Potential regions for the NP contributions in $\Delta C_{1}^{s, c c}\left(M_{W}\right)$ and $\Delta C_{2}^{s, c c}\left(M_{W}\right)$ allowed by the life-time ratio $\tau_{B_{s}} / \tau_{B_{d}}$ at $90 \%$ C.L. . Here we assumed only BSM contributions to the decay channel $b \rightarrow c \bar{c} s$, but none to $b \rightarrow c \bar{u} d$. The black point corresponds to the SM value.

studied in section 4.3.3 for the case of $B_{s} \rightarrow J / \Psi \phi$ and found to give very weak bounds. Thus we will not consider them here. The second effect is also expected to give relatively weak bounds, but since the lack of strong bounds on new contributions to $b \rightarrow c \bar{c} d$ we will consider it here - in the $b \rightarrow c \bar{c} s$ we neglected it, because of much stronger constraints from other observables.

We can constrain $\Delta C_{2}^{d, c c}\left(M_{W}\right)$ with the observable

$$
\sin \left(2 \beta_{d}\right)=-S_{J / \psi K_{S}}
$$

which can be evaluated by applying the generic definition of the CP asymmetry shown in eq. (4.19) and using

$$
\lambda_{J / \psi K_{S}}^{d}=\left.\frac{q}{p}\right|_{B_{d}} \frac{\overline{\mathcal{A}}_{J / \psi K_{S}}}{\mathcal{A}_{J / \psi K_{S}}} .
$$

Where in eq. (4.76), $\mathcal{A}_{J / \psi K_{S}}$ and $\overline{\mathcal{A}}_{J / \psi K_{S}}$ correspond to the amplitudes for the processes $B^{0} \rightarrow J / \psi K_{S}$ and $\bar{B}^{0} \rightarrow J / \psi K_{S}$ respectively.

We study here modifications of $q /\left.p\right|_{B_{d}}$, while we neglect the change of the amplitudes $\mathcal{A}_{J / \psi K_{S}}$ and $\overline{\mathcal{A}}_{J / \psi K_{S}}$ - since an exploratory study found much weaker bounds. The definition of $q /\left.p\right|_{B_{d}}$ in terms of the $B_{d}$ matrix element $M_{12}^{d}$ is given in eq. (4.21).

In the SM we have

$$
M_{12}^{d, \mathrm{SM}}=\frac{\left\langle B_{d}^{0}\left|\hat{\mathcal{H}}_{d}^{|\Delta B|=2, \mathrm{SM}}\right| \bar{B}_{d}^{0}\right\rangle}{2 M_{B_{d}^{0}}},
$$

with

$$
\hat{\mathcal{H}}_{d}^{|\Delta B|=2, \mathrm{SM}}=\frac{G_{F}^{2}}{16 \pi^{2}}\left(\lambda_{t}^{(d)}\right)^{2} C^{|\Delta B|=2}\left(m_{t}, M_{W}, \mu\right) \hat{Q}_{1}^{d}+\text { h.c. } .
$$

The dimension six effective $|\Delta B|=2$ operator $Q_{1}^{d}$ in eq. (4.78) is given by

$$
\hat{Q}_{1}^{d}=(\overline{\hat{d} \hat{b}})_{V-A}(\overline{\hat{d} \hat{b}})_{V-A}
$$

and the Wilson coefficient $C^{|\Delta B|=2}\left(m_{t}, M_{W}, \mu\right)$ corresponds to

$$
C^{|\Delta B|=2}\left(m_{t}, M_{W}, \mu\right)=\tilde{\eta} M_{W}^{2} S_{0}\left(x_{t}\right),
$$




\begin{tabular}{|c|c|c|c|c|}
\hline$\Delta M_{s}^{\mathrm{SM}}$ & This work & ABL 2015 & LN 2011 & LN 2006 \\
\hline Central Value & $18.77 \mathrm{ps}^{-1}$ & $18.3 \mathrm{ps}^{-1}$ & $17.3 \mathrm{ps}^{-1}$ & $19.3 \mathrm{ps}^{-1}$ \\
\hline$f_{B_{s}} \sqrt{B_{1}^{s}}$ & $3.1 \%$ & $13.9 \%$ & $13.5 \%$ & $34.1 \%$ \\
\hline$V_{c b}$ & $3.4 \%$ & $4.9 \%$ & $3.4 \%$ & $4.9 \%$ \\
\hline $\bar{m}_{t}\left(\bar{m}_{t}\right)$ & $0.3 \%$ & $0.7 \%$ & $1.1 \%$ & $1.8 \%$ \\
\hline$\Lambda_{5}^{\mathrm{QCD}}$ & $0.2 \%$ & $0.1 \%$ & $0.4 \%$ & $2.0 \%$ \\
\hline$\gamma$ & $0.1 \%$ & $0.1 \%$ & $0.3 \%$ & $1.0 \%$ \\
\hline$\left|V_{u b} / V_{c b}\right|$ & $<0.1 \%$ & $0.1 \%$ & $0.2 \%$ & $0.5 \%$ \\
\hline $\bar{m}_{b}$ & $<0.1 \%$ & $<0.1 \%$ & $0.1 \%$ & - \\
\hline Total & $4.6 \%$ & $14.8 \%$ & $14.0 \%$ & $34.6 \%$ \\
\hline
\end{tabular}

Table 12. List of the individual contributions to the theoretical error of the mass difference $\Delta M_{s}$ within the Standard Model and comparison with the values obtained in 2015 [31], in 2011 [128] and in 2006 [87].

where the factor $\tilde{\eta}$ accounts for the renormalization group evolution from the scale $m_{t}$ down to the renormalization scale $\mu \sim m_{b}$ [89] and $S_{0}\left(x_{t}\right)$ is the Inami-Lim function [88]

$$
S_{0}\left(x_{t}\right)=\frac{x_{t}}{\left(1-x_{t}\right)^{2}}\left(1-\frac{11}{4} x_{t}+\frac{x_{t}^{2}}{4}-\frac{3 x_{t}^{2} \ln x_{t}}{\left(1-x_{t}\right)}\right)
$$

Using the new averages presented in [65] for the hadronic matrix elements (based on the non-perturbative calculations in [82, 90, 91] and [92-95]) we get the new updated SM results

$$
\begin{aligned}
& \Delta M_{s}^{\mathrm{SM}}=(18.77 \pm 0.86) \mathrm{ps}^{-1} \\
& \Delta M_{d}^{\mathrm{SM}}=(0.543 \pm 0.029) \mathrm{ps}^{-1},
\end{aligned}
$$

where we observe a huge reduction of the theoretical uncertainty, see tables 12 and 13 . Our numbers agree with the ones quoted in [65] - a tiny difference stems from a different treatment of the top quark mass, the CKM input and the symmetrisation of the error we have performed here. HFLAV [111] gives for the experimental values

$$
\begin{aligned}
\Delta M_{s}^{\operatorname{Exp}} & =(17.757 \pm 0.021) \mathrm{ps}^{-1}, \\
\Delta M_{d}^{\exp } & =(0.5064 \pm 0.0019) \mathrm{ps}^{-1} .
\end{aligned}
$$

We introduce BSM effects to eq. (4.77) by adding to the SM expression in eq. (4.78) the double insertion of the effective Hamiltonian

$$
\hat{\mathcal{H}}_{\mathrm{eff}}^{|\Delta B|=1}=\frac{G_{F}}{\sqrt{2}}\left(\sum_{p, p^{\prime}=u, c} \lambda_{p p^{\prime}}^{(d)} C_{2}^{d, p p^{\prime}} \hat{Q}_{2}^{d, p p^{\prime}}+\text { h.c. }\right) .
$$




\begin{tabular}{|c|c|c|}
\hline$\Delta M_{d}^{\mathrm{SM}}$ & This work & ABL 2015 \\
\hline Central Value & $0.543 \mathrm{ps}^{-1}$ & $0.528 \mathrm{ps}^{-1}$ \\
\hline$f_{B_{d}} \sqrt{B_{1}^{d}}$ & $3.6 \%$ & $13.7 \%$ \\
\hline$V_{c b}$ & $3.4 \%$ & $4.9 \%$ \\
\hline$m_{b}$ & $0.1 \%$ & $0.1 \%$ \\
\hline$\gamma$ & $0.2 \%$ & $0.2 \%$ \\
\hline$\Lambda_{5}^{\mathrm{QCD}}$ & $0.2 \%$ & $0.1 \%$ \\
\hline$\left|V_{u b} / V_{c b}\right|$ & $0.1 \%$ & $0.1 \%$ \\
\hline $\bar{m}_{t}\left(\bar{m}_{t}\right)$ & $0.3 \%$ & $0.1 \%$ \\
\hline Total & $5.3 \%$ & $14.8 \%$ \\
\hline
\end{tabular}

Table 13. List of the individual contributions to the theoretical error of the mixing quantity $\Delta M_{d}$ and comparison with the values obtained in 2015 [31].

Following [133] we evaluate the full combination at the scale $\mu_{c}=m_{c}$, where the extra contribution to the SM $|\Delta B|=2$ Hamiltonian in eq. (4.78) is given by

$$
\begin{aligned}
\hat{\mathcal{H}}_{\text {extra }}^{|\Delta B|=2} \approx & \frac{G_{F}^{2}}{16 \pi^{2}}\left\{C_{1}^{\prime}\left(\mu_{c}\right) \hat{P}_{1}+C_{2}^{\prime}\left(\mu_{c}\right) \hat{P}_{2}\right. \\
& \left.+\left[\left(2 \lambda_{c}^{(d)} \lambda_{t}^{(d)} \tilde{C}_{3}\left(x_{t}^{2}\right)+\left(\lambda_{c}^{(d)}\right)^{2}\right)+C_{3}^{\prime}\left(\mu_{c}\right)\right] \hat{P}_{3}\right\},
\end{aligned}
$$

with

$$
\begin{aligned}
C_{1}^{\prime}\left(\mu_{c}\right)= & -\frac{2}{3} \ln \left[\frac{\mu_{c}^{2}}{M_{W}^{2}}\right]\left\{\frac{\left(\lambda_{c}^{(d)}\right)^{2}}{2}\left(C_{2}^{d, c c}\right)^{2}-\left(\lambda_{c}^{(d)}\right)^{2} C_{2}^{d, c u} C_{2}^{d, u c}-\lambda_{c}^{(d)} \lambda_{t}^{(d)} C_{2}^{d, c u} C_{2}^{d, u c}\right. \\
& \left.+\frac{\left(\lambda_{c}^{(d)}\right)^{2}}{2}\left(C_{2}^{d, u u}\right)^{2}+\lambda_{c}^{(d)} \lambda_{t}^{(d)}\left(C_{2}^{d, u u}\right)^{2}+\frac{\left(\lambda_{t}^{(d)}\right)^{2}}{2}\left(C_{2}^{d, u u}\right)^{2}\right\}, \\
C_{2}^{\prime}\left(\mu_{c}\right)= & \frac{2}{3} \ln \left[\frac{\mu_{c}^{2}}{M_{W}^{2}}\right]\left\{\left(\lambda_{c}^{(d)}\right)^{2}\left(C_{2}^{d, c c}\right)^{2}-2\left(\lambda_{c}^{(d)}\right)^{2} C_{2}^{d, c u} C_{2}^{d, u c}-2 \lambda_{c}^{(d)} \lambda_{t}^{(d)} C_{2}^{d, c u} C_{2}^{d, u c}\right. \\
& \left.+\left(\lambda_{c}^{(d)}\right)^{2}\left(C_{2}^{d, u u}\right)^{2}+2 \lambda_{c} \lambda_{t}^{(d)}\left(C_{2}^{d, u u}\right)^{2}+\left(\lambda_{t}^{(d)}\right)^{2}\left(C_{2}^{d, u u}\right)^{2}\right\}, \\
C_{3}^{\prime}\left(\mu_{c}\right)= & \frac{2}{3} \ln \left[\frac{\mu_{c}^{2}}{M_{W}^{2}}\right]\left\{3\left(\lambda_{c}^{(d)}\right)^{2}\left(C_{2}^{d, c c}\right)^{2}-3\left(\lambda_{c}^{(d)}\right)^{2} C_{2}^{d, c u} C_{2}^{d, u c}-3 \lambda_{c}^{(d)} \lambda_{t}^{(d)} C_{2}^{d, c u} C_{2}^{d, u c}\right\},
\end{aligned}
$$

and

$$
\tilde{C}_{3}\left(x_{t}\right)=\ln x_{t}-\frac{3 x_{t}}{4\left(1-x_{t}\right)}-\frac{3 x_{t}^{2} \ln x_{t}}{4\left(1-x_{t}\right)^{2}} .
$$

The set of HQET operators required in eq. (4.87) are

$$
\begin{array}{ll}
\hat{P}_{0}=\left(\overline{\hat{h}}^{(+)} \hat{d}\right)_{V-A}\left(\overline{\hat{h}}^{(-)} \hat{d}\right)_{V-A}, & \hat{P}_{1}=m_{b}^{2} \hat{P}_{0}, \\
\hat{P}_{2}=m_{b}^{2}\left(\overline{\hat{h}}_{v}^{(+)}\left[1-\gamma_{5}\right] \hat{d}\right)\left(\overline{\hat{h}}_{v}^{(-)}\left[1-\gamma_{5}\right] \hat{d}\right), & \hat{P}_{3}=m_{c}^{2} \hat{P}_{0} .
\end{array}
$$




\begin{tabular}{|c|c|}
\hline Parameter & Relative error \\
\hline$\left|V_{u b} / V_{c b}\right|$ & $4.22 \%$ \\
\hline$\left|V_{u s}\right|$ & $0.20 \%$ \\
\hline$\gamma$ & $0.04 \%$ \\
\hline$\mu_{c}$ & $0.02 \%$ \\
\hline$\left|V_{c b}\right|$ & $0.01 \%$ \\
\hline Total & $4.22 \%$ \\
\hline
\end{tabular}

Table 14. Error budget for the observable $\sin \left(2 \beta_{d}\right)$.

Thus, our full determination of $M_{12}^{d}$ is given by

$$
M_{12}^{d}=\frac{\left\langle B_{d}^{0}\left|\hat{\mathcal{H}}_{d}^{|\Delta B|=2, \mathrm{SM}}+\hat{\mathcal{H}}_{\text {extra }}^{|\Delta B|=2}\right| \bar{B}_{d}^{0}\right\rangle}{2 M_{B_{d}^{0}}},
$$

where the $|\Delta B|=2$ operator $\hat{Q}_{1}^{d}$ is matched at the scale $\mu_{c}=m_{c}$ into $\hat{P}_{0}$ [133]. The required matrix elements for the numerical evaluations are [82]

$$
\begin{aligned}
\left\langle B_{d}^{0}\left|\hat{P}_{0}\right| \bar{B}_{d}^{0}\right\rangle & =\frac{8}{3} f_{B_{d}}^{2} M_{B_{d}}^{2} B_{1}^{d}\left(\mu_{c}\right), \\
\left\langle B_{d}^{0}\left|\hat{P}_{2}\right| \bar{B}_{d}^{0}\right\rangle & =-\frac{5}{3} m_{b}^{2}\left(\frac{M_{B_{d}}}{m_{b}+m_{d}}\right)^{2} f_{B_{d}}^{2} M_{B_{d}}^{2} B_{2}^{d}\left(\mu_{c}\right),
\end{aligned}
$$

with the values for the Bag parameters as indicated in appendix A. Our theoretical result - neglecting contributions from penguins - is

$$
\sin \left(2 \beta_{d}^{\mathrm{SM}}\right)=0.707 \pm 0.030,
$$

the full error budget in the SM can be found in table 14. Notice that, the contributions from double insertions of the $|\Delta B|=1$ effective Hamiltonian are relevant only when $\Delta C_{2}^{d, c c}\left(M_{W}\right) \neq 0$, hence they do not appear in table 14. On the experimental side we use the average from direct measurements [111]

$$
\sin \left(2 \beta_{d}^{\operatorname{Exp}}\right)=0.699 \pm 0.017,
$$

our results for the allowed regions on $\Delta C_{2}^{d, c c}\left(M_{W}\right)$ are shown in figure 14 .

\subsection{2 $\quad \bar{B} \rightarrow X_{d} \gamma$}

The branching ratio of the process $\bar{B} \rightarrow X_{d} \gamma$ allows us to impose further constraints on the NP contribution $\Delta C_{2}^{d, c c}\left(M_{W}\right)$. For the theoretical determination, we used the NNLO branching ratio for the transition $\bar{B} \rightarrow X_{d} \gamma$ given in [134]

$$
\mathcal{B}_{r}^{\mathrm{NNLO}}\left(\bar{B} \rightarrow X_{d} \gamma\right)=\left(1.73_{-0.22}^{+0.12}\right) \cdot 10^{-5} \quad \text { for } E_{\gamma}>1.6 \mathrm{GeV} .
$$

On the experimental side we consider [135-137]

$$
\mathcal{B}_{r}^{\operatorname{Exp}}\left(\bar{B} \rightarrow X_{d} \gamma\right)=(1.41 \pm 0.57) \cdot 10^{-5}
$$




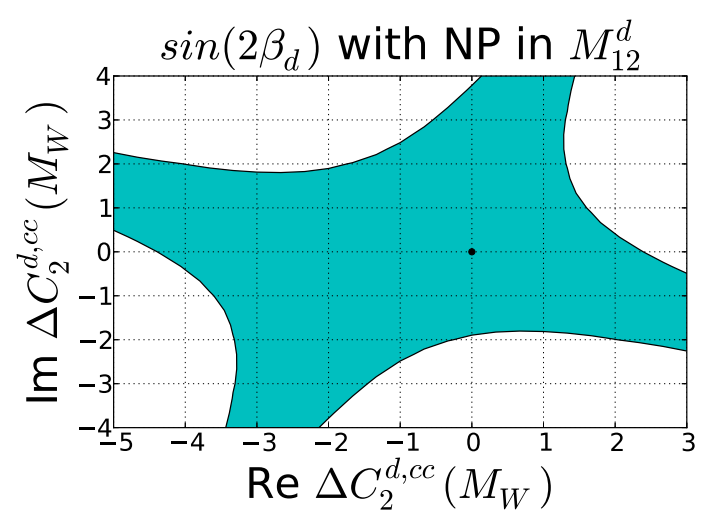

Figure 14. Potential regions for the NP contributions in $\Delta C_{2}^{d, c c}\left(M_{W}\right)$ allowed by the observable $\sin \left(2 \beta_{d}\right)$ from modifications in $M_{12}^{d}$ through double insertions of the $\Delta B=1$ effective Hamiltonian at $90 \%$ C.L. . Due to the weakness of the current bounds, penguin pollution has been neglected in the analysis. The black point corresponds to the SM value.

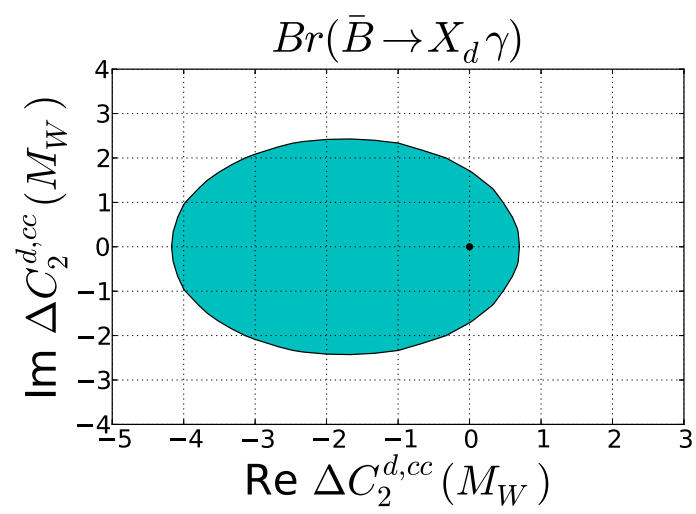

Figure 15. Potential regions for the NP contributions in $\Delta C_{2}^{d, c c}\left(M_{W}\right)$ allowed by the observable $\mathcal{B} r\left(\bar{B} \rightarrow X_{d} \gamma\right)$ at $90 \%$ C.L. . The black point corresponds to the SM value.

The NP regions on $\Delta C_{1}^{c c, d}\left(M_{W}\right)$ derived from $\mathcal{B}_{r}^{\mathrm{NNLO}}\left(\bar{B} \rightarrow X_{d} \gamma\right)$ are shown in figure 15 . Our treatment for $\bar{B} \rightarrow X_{d} \gamma$ is analogous to the one of $\bar{B} \rightarrow X_{s} \gamma$, therefore our discussion here is rather short and we refer the reader to the details provided in section 4.3.1.

\subsection{Observables constraining multiple channels}

Several observables like $\Delta \Gamma_{q}, \tau\left(B_{s}\right) / \tau\left(B_{d}\right)$ and the semi-leptonic CP asymmetries are affected by different decay channels. We have shown that $\Delta \Gamma_{s}$ is by far dominated by the $b \rightarrow c \bar{c} s$ transition, $\Delta \Gamma_{d}$ has not yet been measured. In $\tau\left(B_{s}\right) / \tau\left(B_{d}\right)$ a new effect in the $b \rightarrow c \bar{c} s$ transition roughly cancels a similar size effect in a $b \rightarrow c \bar{u} d$ transition, thus we have assumed for this observable only BSM effects in the $b \rightarrow c \bar{c} s$ transition. Below we will study constraints stemming from $a_{s l}^{q}$, which is affected by the decay channels $b \rightarrow c \bar{c} q$, $b \rightarrow c \bar{u} q, b \rightarrow u \bar{c} q$ and $b \rightarrow u \bar{u} q$. 


\begin{tabular}{|c|c|c|c|c|}
\hline$a_{\mathrm{sl}}^{s, \mathrm{SM}}$ & this work & ABL 2015 & LN 2011 & LN 2006 \\
\hline Central Value & $2.06 \cdot 10^{-5}$ & $2.22 \cdot 10^{-5}$ & $1.90 \cdot 10^{-5}$ & $2.06 \cdot 10^{-5}$ \\
\hline$\mu$ & $6.7 \%$ & $9.5 \%$ & $8.9 \%$ & $12.7 \%$ \\
\hline $\bar{z}$ & $4.0 \%$ & $4.6 \%$ & $7.9 \%$ & $9.3 \%$ \\
\hline$\left|V_{u b} / V_{c b}\right|$ & $2.6 \%$ & $5.0 \%$ & $11.6 \%$ & $19.5 \%$ \\
\hline$B_{R_{3}}^{s}$ & $2.3 \%$ & $1.1 \%$ & $1.2 \%$ & $1.1 \%$ \\
\hline$B_{\tilde{R}_{3}}^{s}$ & - & $2.6 \%$ & $2.8 \%$ & $2.5 \%$ \\
\hline$m_{b}$ & $1.3 \%$ & $1.0 \%$ & $2.0 \%$ & $3.7 \%$ \\
\hline$\gamma$ & $1.1 \%$ & $1.3 \%$ & $3.1 \%$ & $11.3 \%$ \\
\hline$B_{R_{2}}^{s}$ & $0.8 \%$ & $0.1 \%$ & $0.1 \%$ & - \\
\hline$\Lambda_{5}^{\mathrm{QCD}}$ & $0.6 \%$ & $0.5 \%$ & $1.8 \%$ & $0.7 \%$ \\
\hline $\bar{m}_{t}\left(\bar{m}_{t}\right)$ & $0.3 \%$ & $0.7 \%$ & $1.1 \%$ & $1.8 \%$ \\
\hline$B_{3}^{s}$ & $0.3 \%$ & $0.3 \%$ & $0.6 \%$ & $0.4 \%$ \\
\hline$B_{R_{0}}^{s}$ & $0.3 \%$ & $0.2 \%$ & $0.3 \%$ & - \\
\hline$m_{s}$ & $<0.1 \%$ & $0.1 \%$ & $0.1 \%$ & $0.1 \%$ \\
\hline$B_{\tilde{R}_{1}}^{s}$ & $<0.1 \%$ & $0.5 \%$ & $0.2 \%$ & - \\
\hline$B_{R_{1}}^{s}$ & $<0.1 \%$ & $<0.1 \%$ & $0.0 \%$ & - \\
\hline$V_{c b}$ & $<0.1 \%$ & $0.0 \%$ & $0.0 \%$ & $0.0 \%$ \\
\hline Total & $8.8 \%$ & $12.2 \%$ & $17.3 \%$ & $27.9 \%$ \\
\hline & & & & \\
\hline & & & \\
\hline
\end{tabular}

Table 15. List of the individual contributions to the theoretical error of the semileptonic CP asymmetries $a_{s l}^{s}$ within the Standard Model and comparison with the values obtained in 2015 [31], in 2011 [128] and in 2006 [87]. We have used equations of motion in the current analysis to get rid of the operator $\tilde{R}_{3}$.

\subsection{1 $a_{s l}^{s}$ and $a_{s l}^{d}$ : bounds and SM update}

The theoretical description of the semi-leptonic CP asymmetries was already presented in detail in section 4.3.2. Our SM predictions for the semileptonic asymmetries $a_{s l}^{s}$ and $a_{s l}^{d}$ are

$$
\begin{aligned}
& a_{s l}^{s, \mathrm{SM}}=(2.06 \pm 0.18) \cdot 10^{-5}, \\
& a_{s l}^{d, \mathrm{SM}}=(-4.73 \pm 0.42) \cdot 10^{-4} .
\end{aligned}
$$

The error budgets of the mixing observables $a_{s l}^{s}$ and $a_{s l}^{d}$ within the SM are presented in tables 15 and 16 respectively.

The current experimental bounds [111] are far above the SM predictions

$$
\begin{aligned}
& a_{s l}^{s, \operatorname{Exp}}=(60 \pm 280) \cdot 10^{-5}, \\
& a_{s l}^{d, \operatorname{Exp}}=(-21 \pm 17) \cdot 10^{-4} .
\end{aligned}
$$




\begin{tabular}{|c|c|c|}
\hline$a_{\text {sl }}^{d, \text { SM }}$ & This work & ABL 2015 \\
\hline Central Value & $-4.7 \cdot 10^{-4}$ & $-4.7 \cdot 10^{-4}$ \\
\hline$B_{\widetilde{R}_{2}}^{d}$ & $0.8 \%$ & $0.1 \%$ \\
\hline$\mu$ & $6.7 \%$ & $9.4 \%$ \\
\hline$V_{c b}$ & $0.0 \%$ & $0.0 \%$ \\
\hline$B_{3}^{d}$ & $0.4 \%$ & $0.6 \%$ \\
\hline$B_{R_{0}}^{d}$ & $0.3 \%$ & $0.2 \%$ \\
\hline $\bar{z}$ & $4.1 \%$ & $4.9 \%$ \\
\hline$m_{b}$ & $1.3 \%$ & $1.3 \%$ \\
\hline$B_{\tilde{R}_{3}}^{d}$ & $-\%$ & $2.7 \%$ \\
\hline$B_{R_{3}}^{d}$ & $2.3 \%$ & $1.2 \%$ \\
\hline$\gamma$ & $1.0 \%$ & $1.1 \%$ \\
\hline$\Lambda_{5}^{\mathrm{QCD}}$ & $0.8 \%$ & $0.5 \%$ \\
\hline $\mid V_{u b} / V_{c b}$ & $2.7 \%$ & $5.2 \%$ \\
\hline $\bar{m}_{t}\left(\bar{m}_{t}\right)$ & $0.3 \%$ & $0.7 \%$ \\
\hline Total & $8.8 \%$ & $12.3 \%$ \\
\hline
\end{tabular}

Table 16. List of the individual contributions to the theoretical error of the mixing quantity $a_{\mathrm{sl}}^{d, \mathrm{SM}}$ in the $B^{0}$-sector and comparison with the values obtained in 2015 [31]. We have used equations of motion in the current analysis to get rid of the operator $\tilde{R}_{3}$.

Nevertheless, these observables yield already, with the current experimental precision, strong bounds on $C_{1}$ and $C_{2}$ due to the pronounced sensitivity of $\operatorname{Im}\left(\Gamma_{12}^{q} / M_{12}^{q}\right)$ on the imaginary components of the $\Delta B=1$ Wilson coefficients. The regions for $\Delta C_{1}\left(M_{W}\right)$ and $\Delta C_{2}\left(M_{W}\right)$ allowed by the observables $a_{s l}^{s}$ and $a_{s l}^{d}$ are presented in figures 16 and 17 respectively where for simplicity we have assumed the universal behaviour

$$
\Delta C_{j}^{q, u u}\left(M_{W}\right)=\Delta C_{j}^{q, u c}\left(M_{W}\right)=\Delta C_{j}^{q, c c}\left(M_{W}\right),
$$

for $j=1,2$. As discussed in section 4.3.2 different BSM effects in individual decay channels could lift the severe GIM suppression and lead to large effects, while the scenario given in eq. (4.100) is dominated by $b \rightarrow c \bar{c} q$ transitions. However, in sections 5.1, 5.2 and 4.4 we will also study the effects of $a_{s l}^{d}$ on the different $b$-quark decay channels $b \rightarrow u \bar{u} d, b \rightarrow c \bar{u} d$, and $b \rightarrow c \bar{c} d$ independently.

\section{$5 \quad$ Global $\chi^{2}$-fit results}

So far, we have limited our discussion to constraints derived from individual observables. In this section, we present, as the main result of this work, the resulting regions for $\Delta C_{1}\left(M_{W}\right)$ and $\Delta C_{2}\left(M_{W}\right)$ obtained after combining observables for the different exclusive $b$ quark 

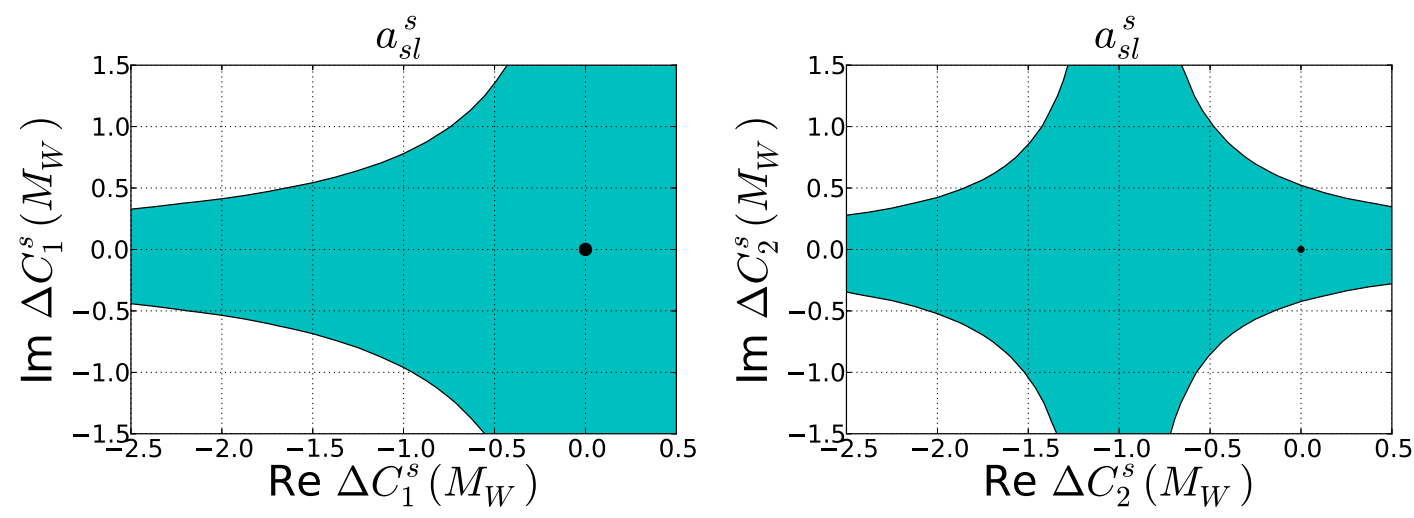

Figure 16. Potential regions for the NP contributions in $\Delta C_{1}^{s}\left(M_{W}\right)$ and $\Delta C_{2}^{s}\left(M_{W}\right)$ allowed by the semileptonic asymmetry $a_{s l}^{s}$ at $90 \%$ C.L. . The black point corresponds to the SM value. For the purposes of illustration we have made the universality assumptions: $\Delta C_{1}^{s, u u}\left(M_{W}\right)=\Delta C_{1}^{s, c u}\left(M_{W}\right)=$ $\Delta C_{1}^{s, u c}\left(M_{W}\right)=\Delta C_{1}^{s, c c}\left(M_{W}\right)=\Delta C_{1}^{s}\left(M_{W}\right)$ and similarly for $\Delta C_{2}^{s}\left(M_{W}\right)$.
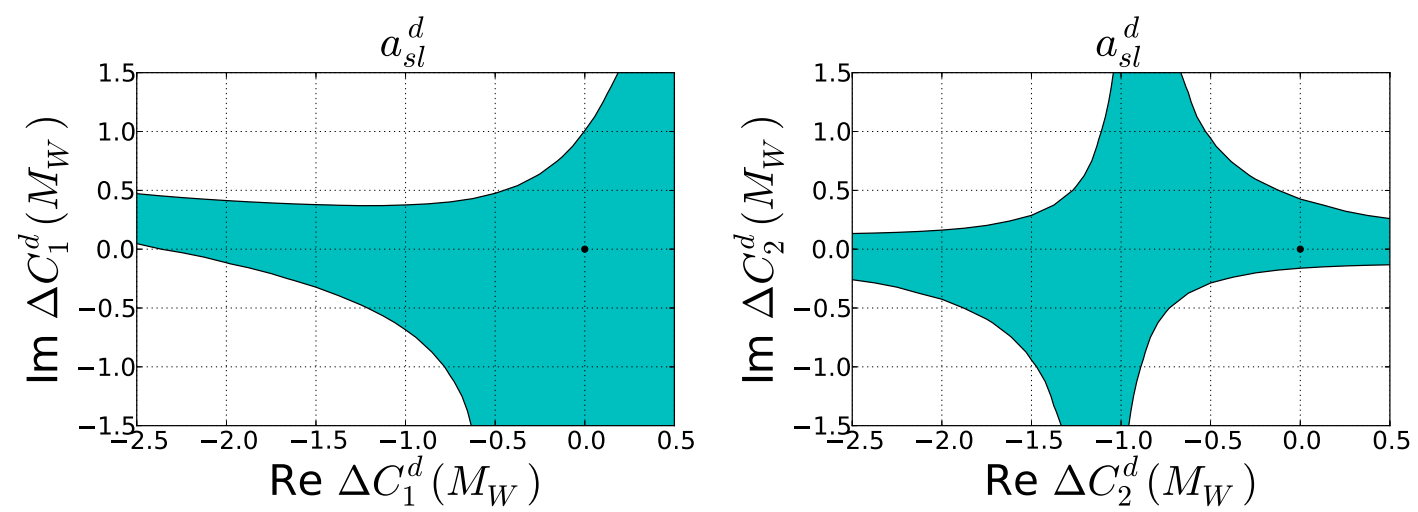

Figure 17. Potential regions for the NP contributions in $\Delta C_{1}^{d}\left(M_{W}\right)$ and $\Delta C_{2}^{d}\left(M_{W}\right)$ allowed by the semileptonic asymmetry $a_{s l}^{d}$ at $90 \%$ C.L. . The black point corresponds to the SM value. For the purposes of illustration we have made the universality assumptions: $\Delta C_{1}^{d, u u}\left(M_{W}\right)=\Delta C_{1}^{d, c u}\left(M_{W}\right)=$ $\Delta C_{1}^{d, u c}\left(M_{W}\right)=\Delta C_{1}^{d, c c}\left(M_{W}\right)=\Delta C_{1}^{d}\left(M_{W}\right)$ and similarly for $\Delta C_{2}^{d}\left(M_{W}\right)$.

transitions. We will investigate three consequences of BSM effects in non-leptonic treelevel decays.

1. The allowed size of BSM contributions to the Wilson coefficients $C_{1}$ and $C_{2}$, governing the leading tree-level decays.

2. The impact of these new effects on the possible size of the observable $\Delta \Gamma_{d}$, which has not been measured yet. Notice that, if one sigma deviations are considered, the current experimental uncertainty associated with $\Delta \Gamma_{d}$, see eq. (4.62), allows enhancement factors within the interval

$$
-3.40<\Delta \Gamma_{d}^{\mathrm{Exp}} / \Delta \Gamma_{d}^{\mathrm{SM}}<2.27
$$

On the other hand, if the confidence interval is increased up to 1.65 sigmas, i.e. $90 \%$ 
C.L., then the potential effects in $\Delta \Gamma_{d}$ become

$$
-5.97<\Delta \Gamma_{d}^{\mathrm{Exp}} / \Delta \Gamma_{d}^{\mathrm{SM}}<4.67 .
$$

The measured value of the dimuon asymmetry by the D0-collaboration [33-36] seems to be in conflict with the current experimental bounds on $a_{s l}^{d}$ and $a_{s l}^{s}$, see e.g. the discussion in [138]. An enhanced value of $\Delta \Gamma_{d}$ could solve this experimental discrepancy [139], at the expense of introducing new physics in $\Delta \Gamma_{d}$ and potentially also in $a_{s l}^{s}$ and $a_{s l}^{d}$. If all BSM effects in the dimuon asymmetry are due to $\Delta \Gamma_{d}$, then an enhancement factor of 6 with respect to its SM value is required. On the other hand, if there are also BSM contributions in $a_{s l}^{s}$ and $a_{s l}^{d}$, then the BSM enhancement factor in $\Delta \Gamma_{d}$ can be smaller.

3. The impact of these new effects on the determination of the CKM angle $\gamma$. Within the $\mathrm{SM}$, this quantity can be extracted with negligible uncertainties from $B \rightarrow D K$ treelevel decays [140-145]. This quantity is currently extensively tested by experiments, see e.g. $[146,147]$ and future measurements will dramatically improve its precision to the one degree level [148]. This observable is particular interesting since direct measurements, e.g. LHCb [146], seem to be larger than bounds from B-mixing [64]. ${ }^{7}$

$$
\begin{aligned}
& \gamma^{\mathrm{LHCb}}=\left(74.0_{-5.8}^{+5.0}\right)^{\circ}, \\
& \gamma^{\mathrm{B}-\text { mixing }} \leq 66.9^{\circ} .
\end{aligned}
$$

Therefore, in sections 5.1 to 5.3 we combine our bounds from the $b \rightarrow u \bar{u} d, b \rightarrow c \bar{u} d$ and $b \rightarrow c \bar{c} d$ transitions, and evaluate the corresponding potential enhancement in $\Delta \Gamma_{d}$. We do not present the allowed regions for the NP contributions related to the channel $b \rightarrow u \bar{c} d$, since the bounds are expected to be rather weak considering that our only bound will arise from $a_{s l}^{d}$. In section 5.4 we report the maximal bounds on $\Delta C_{1}\left(M_{W}\right)$ and $\Delta C_{2}\left(M_{W}\right)$, assuming universal BSM contributions to all different quark level decays. Hence, we combine all our possible bounds regardless of the quark level transition and asses the implications on the measurement of the CKM angle $\gamma$. The target of this part of analysis, is to update the investigations reported in [57] in the light of a far more detailed study of BSM effects in non-leptonic tree-level decays. In particular we account here for uncertainties neglected in the former study and we also make a very careful choice of reliable observables.

\section{1 $\chi^{2}$-fit for the $b \rightarrow u \bar{u} d$ channel and bounds on $\Delta \Gamma_{d}$}

We perform a combined $\chi^{2}$-fit including $R_{\pi \pi}, S_{\pi \pi}, S_{\rho \pi}, R_{\rho \rho}$ and $a_{s l}^{d}$ with the aim of constraining $\Delta C_{1}^{d, u u}\left(M_{W}\right)$ and $\Delta C_{2}^{d, u u}\left(M_{W}\right)$. The resulting regions are shown in figure 18. $\Delta C_{2}^{d, u u}\left(M_{W}\right)$ is considerably stronger constrained than $\Delta C_{1}^{d, u u}\left(M_{W}\right)$, but sizeable deviations can still not be excluded. Due to the irregularity of the regions for $\Delta C_{1}^{d, u u}\left(M_{W}\right)$ and $\Delta C_{2}^{d, u u}\left(M_{W}\right)$, expressing the possible NP values for the tree level contributions in terms

\footnotetext{
${ }^{7}$ Similar observations were made in e.g. $[149,150]$.
} 

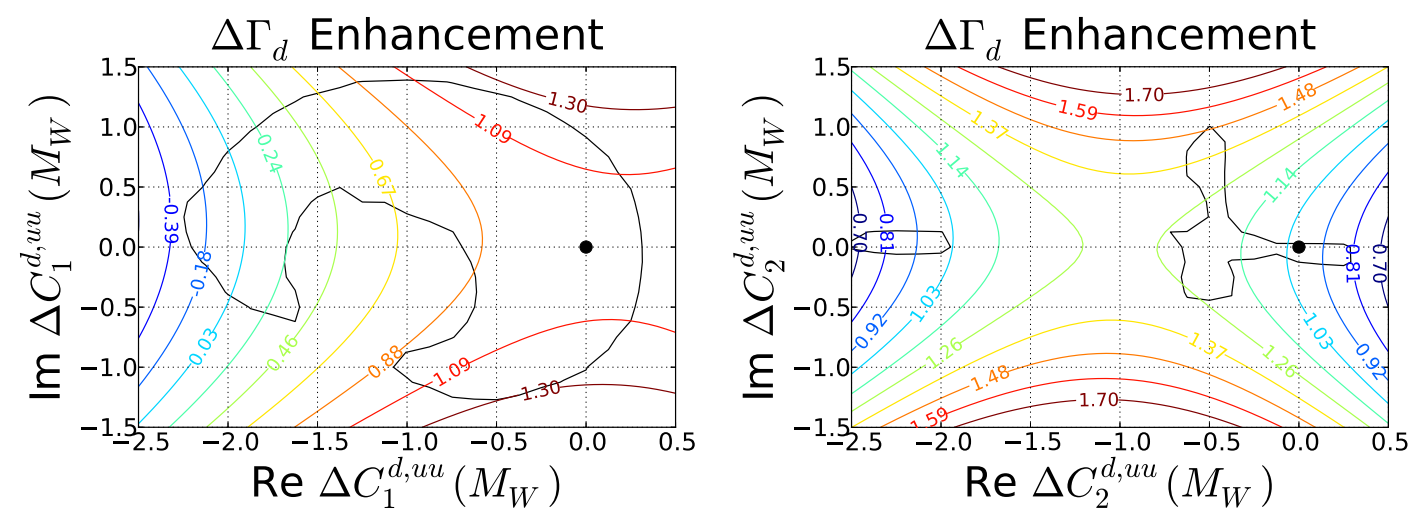

Figure 18. Global $\chi^{2}$-fit including observables constraining the inclusive transition $b \rightarrow u \bar{u} d$. The $90 \%$ C.L. allowed regions correspond to the areas contained within the black contours. The colored curves indicate the possible enhancements on $\Delta \Gamma_{d}$ with respect to the SM value. The black dot corresponds to the SM result.

of simple inequalities is not possible. Instead, we limit ourselves to quote the minimum and maximum bounds for the real and the imaginary components of our NP regions. For $\Delta C_{1}^{d, u u}\left(M_{W}\right)$ we have

$$
\begin{array}{ll}
\left.\operatorname{Re}\left[\Delta C_{1}^{d, u u}\left(M_{W}\right)\right]\right|_{\min }=-2.23, & \left.\operatorname{Im}\left[\Delta C_{1}^{d, u u}\left(M_{W}\right)\right]\right|_{\min }=-1.27, \\
\left.\operatorname{Re}\left[\Delta C_{1}^{d, u u}\left(M_{W}\right)\right]\right|_{\max }=0.32, & \left.\operatorname{Im}\left[\Delta C_{1}^{d, u u}\left(M_{W}\right)\right]\right|_{\max }=1.40 .
\end{array}
$$

On the other hand for $\Delta C_{2}^{d, u u}\left(M_{W}\right)$ we get

$$
\begin{array}{lc}
\left.\operatorname{Re}\left[\Delta C_{2}^{d, u u}\left(M_{W}\right)\right]\right|_{\min }=-2.5, & \left.\operatorname{Im}\left[\Delta C_{2}^{d, u u}\left(M_{W}\right)\right]\right|_{\min }=-0.44, \\
\left.\operatorname{Re}\left[\Delta C_{2}^{d, u u}\left(M_{W}\right)\right]\right|_{\max }=0.28, & \left.\operatorname{Im}\left[\Delta C_{2}^{d, u u}\left(M_{W}\right)\right]\right|_{\max }=1.00 .
\end{array}
$$

We have also included the contour lines showing the potential enhancement of the observable $\Delta \Gamma_{d}$. Accounting for the uncertainties in theory and experiment we find the following $90 \%$ C.L. intervals for $\Delta \Gamma_{d}$ due to NP at tree level:

$$
\begin{array}{lc}
\text { for } \Delta C_{1}^{d, u u}\left(M_{W}\right): & -0.39<\Delta \Gamma_{d} / \Delta \Gamma_{d}^{\mathrm{SM}}<1.30, \\
\text { for } \Delta C_{2}^{d, u u}\left(M_{W}\right): & 0.70<\Delta \Gamma_{d} / \Delta \Gamma_{d}^{\mathrm{SM}}<1.48 .
\end{array}
$$

Thus only moderate enhancements of $\Delta \Gamma_{d}$ seem to be possible, while a reduction to up to $-39 \%$ of its SM values is still possible. This scenario could thus not be a solution for the dimuon asymmetry. 

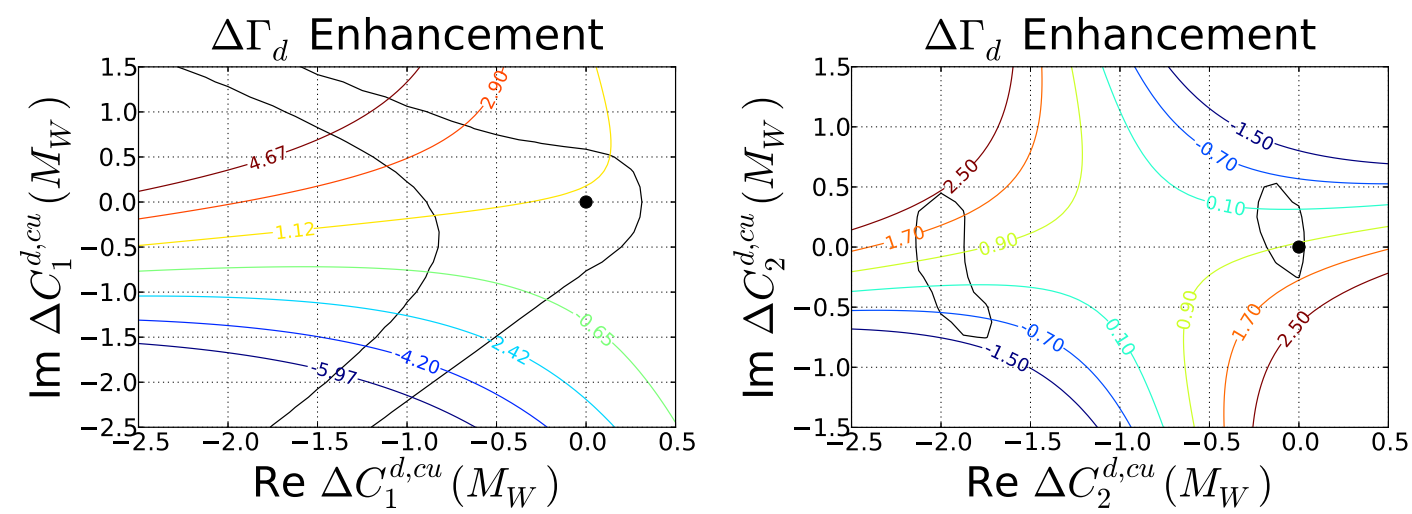

Figure 19. Global $\chi^{2}$-fit including observables constraining the inclusive transition $b \rightarrow c \bar{u} d$. The $90 \%$ C.L. allowed regions correspond to the areas contained within the black contours. The colored curves indicate the possible enhancements on $\Delta \Gamma_{d}$ with respect to the SM value. The black dot corresponds to the SM result.

\section{$5.2 \quad \chi^{2}$-fit for the $b \rightarrow c \bar{u} d$ channel and bounds on $\Delta \Gamma_{d}$}

To establish constraints on $\Delta C_{1}^{d, c u}\left(M_{W}\right)$ and $\Delta C_{2}^{d, c u}\left(M_{W}\right)$ we combine $R_{D^{*} \pi}$ together with $a_{s l}^{d}$. Our results are presented in figure 19. At the $90 \%$ C.L. we find the possibility of huge enhancements/reductions of $\Delta \Gamma_{d}$ :

$$
\begin{array}{ll}
\text { for } \Delta C_{1}^{d, c u}\left(M_{W}\right): & -5.97<\Delta \Gamma_{d} / \Delta \Gamma_{d}^{\mathrm{SM}}<4.67, \\
\text { for } \Delta C_{2}^{d, c u}\left(M_{W}\right): & -1.5<\Delta \Gamma_{d} / \Delta \Gamma_{d}^{\mathrm{SM}}<2.50 .
\end{array}
$$

Based on the bounds shown in eq. (5.8), we find that this scenario could solve the dimuon asymmetry. Since the experimental bounds for $\Delta \Gamma_{d}$ are saturated in the case of $\Delta C_{1}^{d, c u}\left(M_{W}\right)$ in eq. (5.8), it turns out that $\Delta \Gamma_{d}$ acts as a constraint in itself. Using this additional information we establish the following bounds for $\Delta C_{1}^{d, c u}\left(M_{W}\right)$

$$
\begin{array}{ll}
\left.\operatorname{Re}\left[\Delta C_{1}^{d, c u}\left(M_{W}\right)\right]\right|_{\min }=-1.40, & \left.\operatorname{Im}\left[\Delta C_{1}^{d, c u}\left(M_{W}\right)\right]\right|_{\min }=-2.17, \\
\left.\operatorname{Re}\left[\Delta C_{1}^{d, c u}\left(M_{W}\right)\right]\right|_{\max }=0.32, & \left.\operatorname{Im}\left[\Delta C_{1}^{d, c u}\left(M_{W}\right)\right]\right|_{\max }=1.15 .
\end{array}
$$

The corresponding bounds for $\Delta C_{2}^{d, c u}\left(M_{W}\right)$ read

$$
\begin{array}{ll}
\left.\operatorname{Re}\left[\Delta C_{2}^{d, c u}\left(M_{W}\right)\right]\right|_{\min }=-2.14, & \left.\operatorname{Im}\left[\Delta C_{2}^{d, c u}\left(M_{W}\right)\right]\right|_{\min }=-0.75, \\
\left.\operatorname{Re}\left[\Delta C_{2}^{d, c u}\left(M_{W}\right)\right]\right|_{\max }=0.04, & \left.\operatorname{Im}\left[\Delta C_{2}^{d, c u}\left(M_{W}\right)\right]\right|_{\max }=0.53 .
\end{array}
$$

\section{$5.3 \quad \chi^{2}$-fit for the $b \rightarrow c \bar{c} d$ channel and bounds on $\Delta \Gamma_{d}$}

Next we perform a $\chi^{2}$-fit including $\mathcal{B} r\left(B \rightarrow X_{d} \gamma\right), a_{s l}^{d}$ and $\sin \left(2 \beta_{d}\right)$. These observables give strong constraints for $\Delta C_{2}^{d, c c}\left(M_{W}\right)$ (see figure 20), which turn out to saturate the current 

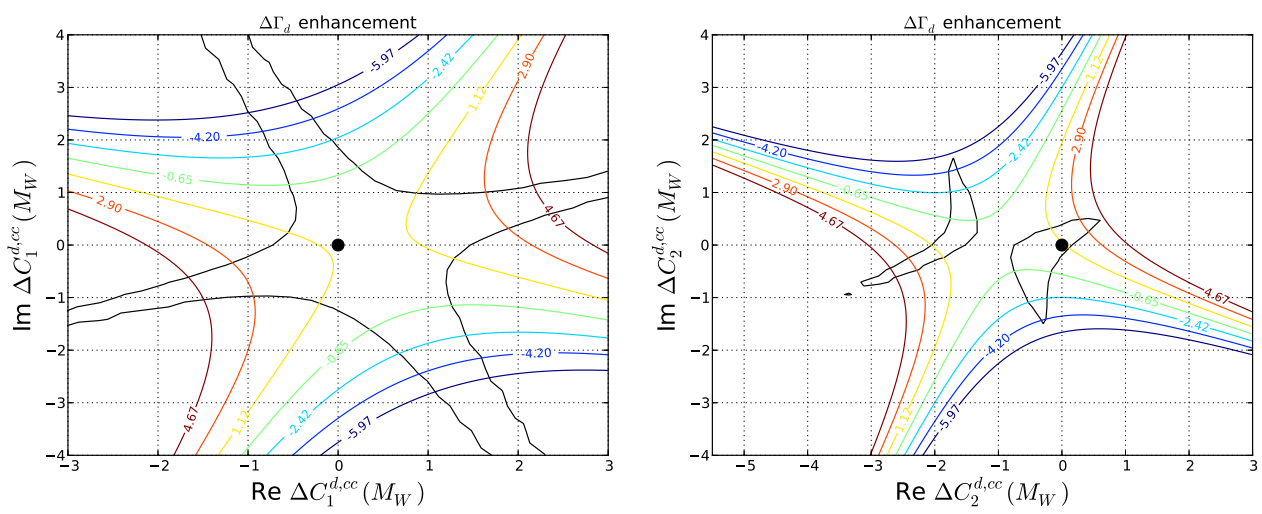

Figure 20. Global $\chi^{2}$-fit including observables constraining the inclusive transition $b \rightarrow c \bar{c} d$. The $90 \%$ C.L. allowed regions correspond to the areas contained within the black contours. The colored curves indicate the possible enhancements on $\Delta \Gamma_{d}$ with respect to the SM value. The black dot corresponds to the SM result.

experimental bounds on $\Delta \Gamma_{d}$. At the $90 \%$ C.L. we find

$$
\text { for } \Delta C_{1}^{d, c c}\left(M_{W}\right) \text { and } \Delta C_{2}^{d, c c}\left(M_{W}\right): \quad-5.97<\Delta \Gamma_{d} / \Delta \Gamma_{d}^{\mathrm{SM}}<4.67 \text {. }
$$

We find again that this scenario could solve the tension between theory and experiment found in the measurement of the dimuon asymmetry. Considering the results shown in figure 20 we see that $\Delta \Gamma_{d}$ is indeed a powerful constraint for $\Delta C_{1}^{d, c c}\left(M_{W}\right)$ and $\Delta C_{2}^{d, c c}\left(M_{W}\right)$, which together with $\mathcal{B} r\left(B \rightarrow X_{d} \gamma\right), a_{s l}^{d}$ and $\sin \left(2 \beta_{d}\right)$ defines the following limits

$$
\begin{array}{ll}
\left.\operatorname{Re}\left[\Delta C_{1}^{d, c c}\left(M_{W}\right)\right]\right|_{\min }=-1.66, & \left.\operatorname{Im}\left[\Delta C_{1}^{d, c c}\left(M_{W}\right)\right]\right|_{\min }=-2.80, \\
\left.\operatorname{Re}\left[\Delta C_{1}^{d, c c}\left(M_{W}\right)\right]\right|_{\max }=2.36, & \left.\operatorname{Im}\left[\Delta C_{1}^{d, c c}\left(M_{W}\right)\right]\right|_{\max }=2.74,
\end{array}
$$

and

$$
\begin{array}{ll}
\left.\operatorname{Re}\left[\Delta C_{2}^{d, c c}\left(M_{W}\right)\right]\right|_{\min }=-2.70, & \left.\operatorname{Im}\left[\Delta C_{2}^{d, c c}\left(M_{W}\right)\right]\right|_{\min }=-1.46, \\
\left.\operatorname{Re}\left[\Delta C_{2}^{d, c c}\left(M_{W}\right)\right]\right|_{\max }=0.58, & \left.\operatorname{Im}\left[\Delta C_{2}^{d, c c}\left(M_{W}\right)\right]\right|_{\max }=1.65 .
\end{array}
$$

As can be seen on the l.h.s. of figure $20 \Delta C_{1}^{d, c c}\left(M_{W}\right)$ is only weakly constrained by the semi-leptonic CP asymmetries, here additional information stemming from $\Delta \Gamma_{d}$ will be important to shrink the allowed regions.

\subsection{Universal fit on $\Delta C_{1}\left(M_{W}\right)$ and $\Delta C_{2}\left(M_{W}\right)$}

In this section we work under the assumptions

$$
\begin{aligned}
& \Delta C_{1}^{s, a b}\left(M_{W}\right)=\Delta C_{1}^{d, a b}\left(M_{W}\right)=\Delta C_{1}\left(M_{W}\right) \\
& \Delta C_{2}^{s, a b}\left(M_{W}\right)=\Delta C_{2}^{d, a b}\left(M_{W}\right)=\Delta C_{2}\left(M_{W}\right)
\end{aligned}
$$



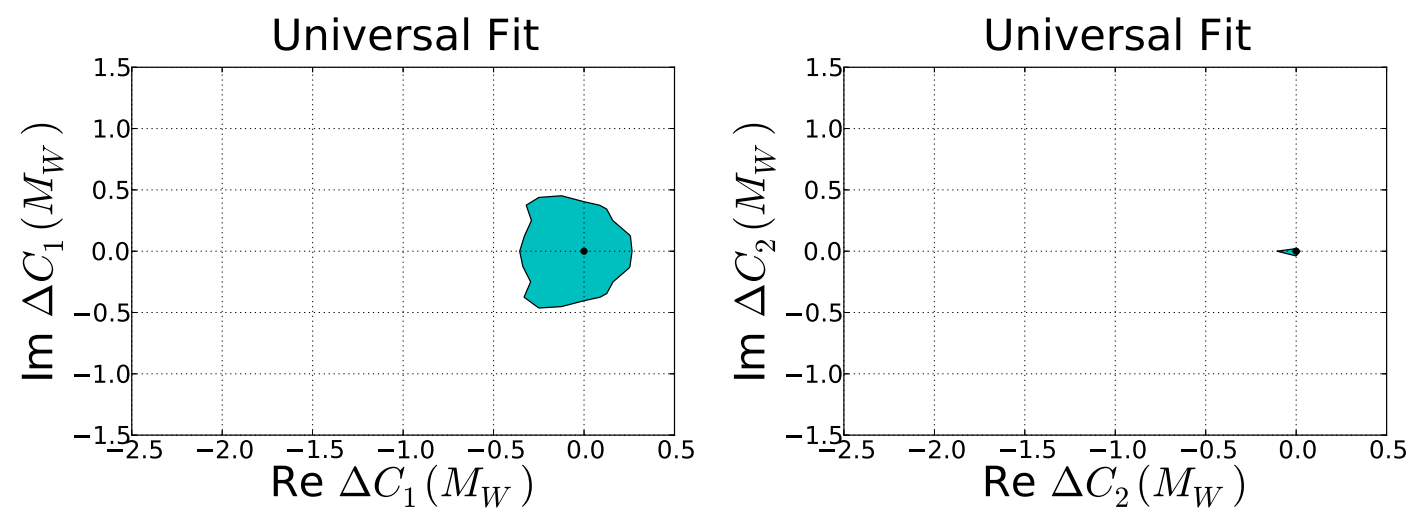

Figure 21. Potential regions for the NP contributions $\Delta C_{1}\left(M_{W}\right)$ and $\Delta C_{2}\left(M_{W}\right)$ allowed by the observables used in our analysis at 90\% C.L. assuming universal NP contributions. The black dot corresponds to the SM result.

for $a=u, d$ and $b=u, d$. This procedure allows us to obtain the maximal constraints for our NP contributions. Making a combined $\chi^{2}$-fit is time and resource consuming, consequently we select the set of observables that give the strongest possible bounds. For $\Delta C_{1}\left(M_{W}\right)$ this includes: $R_{D^{*} \pi}, S_{\rho \pi}, \Delta \Gamma_{s}, \mathcal{B} r\left(\bar{B} \rightarrow X_{s} \gamma\right)$ and $a_{s l}^{d}$ and for $\Delta C_{2}\left(M_{W}\right)$ we use: $R_{D^{*} \pi}, R_{\pi \pi}, \Delta \Gamma_{s}, S_{J / \psi \phi}$ and $\tau_{B_{s}} / \tau_{B_{d}}$. We show in figure 21 our resulting regions from which we extract

$$
\begin{array}{ll}
\left.\operatorname{Re}\left[\Delta C_{1}\left(M_{W}\right)\right]\right|_{\min }=-0.36, & \left.\operatorname{Im}\left[\Delta C_{1}\left(M_{W}\right)\right]\right|_{\min }=-0.47, \\
\left.\operatorname{Re}\left[\Delta C_{1}\left(M_{W}\right)\right]\right|_{\max }=0.26, & \left.\operatorname{Im}\left[\Delta C_{1}\left(M_{W}\right)\right]\right|_{\max }=0.45,
\end{array}
$$

and

$$
\begin{array}{ll}
\left.\operatorname{Re}\left[\Delta C_{2}\left(M_{W}\right)\right]\right|_{\min }=-0.11, & \left.\operatorname{Im}\left[\Delta C_{2}\left(M_{W}\right)\right]\right|_{\min }=-0.04, \\
\left.\operatorname{Re}\left[\Delta C_{2}\left(M_{W}\right)\right]\right|_{\max }=0.02, & \left.\operatorname{Im}\left[\Delta C_{2}\left(M_{W}\right)\right]\right|_{\max }=0.02 .
\end{array}
$$

We can see from eqs. (5.16) and (5.17) how severely constrained is $\Delta C_{2}\left(M_{W}\right)$ allowing deviations with respect to the SM point of a few percent at most. This behaviour is clearly in contrast with the results obtained for $\Delta C_{1}\left(M_{W}\right)$, where effects of almost up to \pm 0.5 are still possible. For completeness we present the implications of universal NP in $\Delta C_{1}\left(M_{W}\right)$ on $\Delta \Gamma_{d}$ in figure 22 . We find that at $90 \%$ C.L. only $\mathcal{O}(20 \%)$ deviations on $\Delta \Gamma_{d}$ with respect to its $\mathrm{SM}$ value can be induced, which is in a similar ballpark as the SM uncertainties of $\Delta \Gamma_{d}$ and can clearly not explain the D0 measurement of the dimuon asymmetry. 


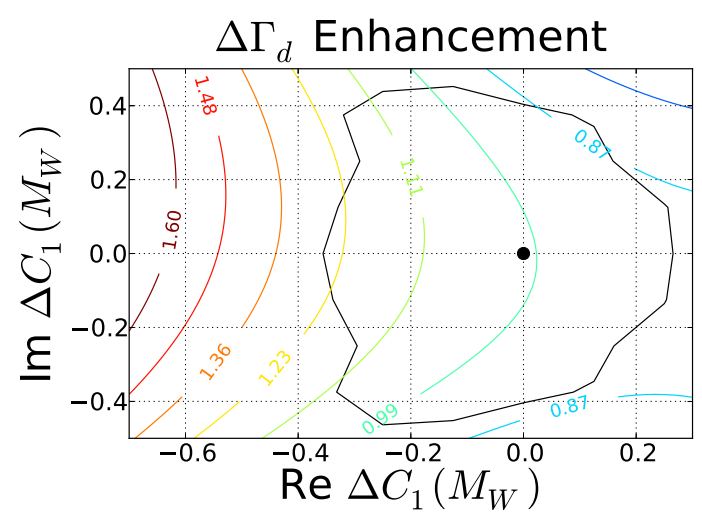

Figure 22. Enhancements on $\Delta \Gamma_{d}$ when assuming universal NP effects in $C_{1}\left(M_{W}\right)$. The black dot corresponds to the SM result.

\subsection{NP in non-leptonic tree-level decays and its interplay with the CKM angle $\gamma$}

As is well known [140-145] the CKM phase $\gamma$ can be determined from the interference of the transition amplitudes associated with the quark tree level decays $b \rightarrow c \bar{u} s$ and $b \rightarrow u \bar{c} s$ with negligible theory uncertainty within the SM [151]. ${ }^{8}$ At the exclusive level, this can be done with the decay channels $B^{-} \rightarrow D^{0} K^{-}$and $B^{-} \rightarrow \bar{D}^{0} K^{-}$. The ratio of the two corresponding decay amplitudes can be written as

$$
r_{B} e^{i\left(\delta_{B}-\gamma\right)}=\frac{\mathcal{A}\left(B^{-} \rightarrow \bar{D}^{0} K^{-}\right)}{\mathcal{A}\left(B^{-} \rightarrow D^{0} K^{-}\right)}
$$

where the $r_{B}$ stands for the ratio of the modulus of the relevant amplitudes. The resulting phase has a strong component, denoted as $\delta_{B}$, and a weak one, which is precisely CKM $\gamma$. New effects in $C_{1}$ and $C_{2}$ can lead to huge shifts in $\gamma$. To study this possibility we follow [152] and assume universal NP in $C_{1}$ and $C_{2}$. Thus $\Delta C_{1}^{s, u c}=\Delta C_{1}^{s, c u}$ and $\Delta C_{2}^{s, u c}=\Delta C_{2}^{s, c u}$. Then, the left side of eq. (5.18) will be modified according to [57]

$$
\begin{aligned}
& r_{B} e^{i\left(\delta_{B}-\gamma\right)} \rightarrow r_{B} e^{i\left(\delta_{B}-\gamma\right)} \cdot\left[\frac{C_{2}+\Delta C_{2}+r_{A^{\prime}}\left(C_{1}+\Delta C_{1}\right)}{C_{2}+r_{A^{\prime}} C_{1}}\right. \\
&\left.\cdot \frac{C_{2}+r_{A} C_{1}}{C_{2}+\Delta C_{2}+r_{A}\left(C_{1}+\Delta C_{1}\right)}\right],
\end{aligned}
$$

where

$$
r_{A^{\prime}}=\frac{\left\langle\bar{D}^{0} K^{-}\left|Q_{1}^{\bar{u} c s}\right| B^{-}\right\rangle}{\left\langle\bar{D}^{0} K^{-}\left|Q_{2}^{\bar{u} c s}\right| B^{-}\right\rangle}, \quad r_{A}=\frac{\left\langle D^{0} K^{-}\left|Q_{1}^{\bar{c} u s}\right| B^{-}\right\rangle}{\left\langle D^{0} K^{-}\left|Q_{2}^{\bar{c} u s}\right| B^{-}\right\rangle}
$$

\footnotetext{
${ }^{8}$ Due to the absence of penguins and the fact that the relevant hadronic matrix elements cancel, the extraction of CKM $\gamma$ is extremely clean. The irreducible theoretical uncertainty is due to higher-order electroweak corrections and has been found to be negligible. For instance, when the modes $B \rightarrow D K$ are used the correction effect is $|\delta \gamma / \gamma|<\mathcal{O}\left(10^{-7}\right)$ [151]. On the other hand, if CKM $\gamma$ is obtained using $B \rightarrow D \pi$ decays instead, then $|\delta \gamma / \gamma|<\mathcal{O}\left(10^{-4}\right)[152]$.
} 

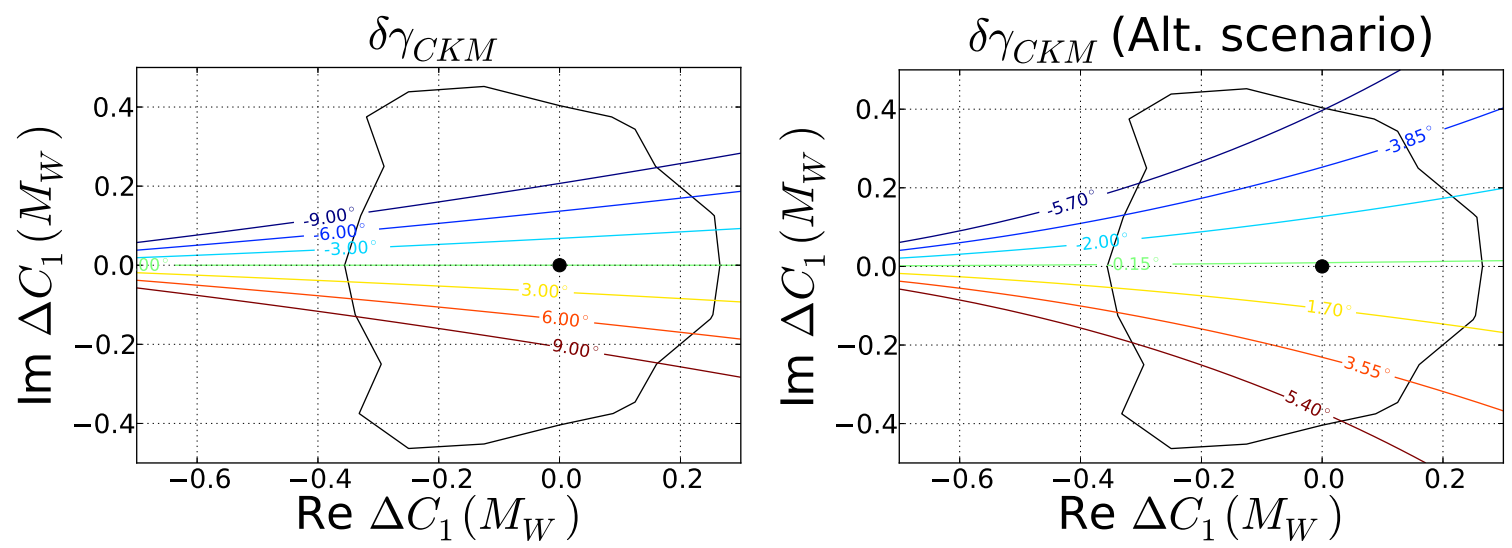

Figure 23. Possible deviations on the CKM phase $\gamma$ due to NP at tree level in $C_{1}\left(M_{W}\right)$ assuming $r_{A}=0.4$ (left) and $r_{A}=0.8$ (right). In both cases we have considered $r_{A}^{\prime}=1$. The black dot corresponds to the SM result.

The ratios of matrix elements in eq. (5.20) have not been determined from first principles, to provide an estimation we use naive factorization arguments and colour counting to obtain $[57,152]$

$$
r_{A}=0.4, \quad r_{A}-r_{A}^{\prime}=-0.6 .
$$

Eq. (5.19) gives a particularly strong dependence of the shift in $\gamma$ on the imaginary part of $C_{1}$; approximately we get [57]

$$
\delta \gamma=\left(r_{A}-r_{A^{\prime}}\right) \frac{\operatorname{Im}\left[\Delta \mathrm{C}_{1}\right]}{C_{2}} .
$$

We are now ready to update the study presented in [57] on the effects of NP in $C_{1}$ and $C_{2}$ on the precision for the determination of the CKM angle $\gamma$, our results are presented graphically in figure 23. On the left hand side of figure 23 we can see how for the values of $r_{A}$ and $r_{A}^{\prime}$ shown in eq. (5.21), the current uncertainties in our knowledge of $C_{1}$ seem to indicate an uncertainty in the extraction of the CKM angle $\gamma$ of considerably more than $10^{\circ}$. This is much higher than the current experimental uncertainty of around five degrees $[146,147]$. Interestingly direct measurements give typically larger values than the ones obtained by CKM fits [153, 154] or extracted from B-mixing [64]. Even more interestingly, future measurements will dramatically improve the precision of $\gamma$ to the one degree level [148] and our BSM approach would offer a possibility of explaining large deviations in the extraction of the CKM angle $\gamma$. We would like, however, to add some words of cautions: for a quantitative reliable relation between the deviations of $C_{1}$ and the shifts in the CKM angle $\gamma$, the non-perturbative parameter $r_{A}$ and $r_{A^{\prime}}$ have to be known more precisely. The values proposed in eq. (5.21) correspond to an educated ansatz. We can explore the effects of modifying these values on CKM- $\gamma$. For instance, consider an alternative scenario where $r_{A}$ is twice the value presented in eq. (5.21), while $r_{A}^{\prime}$ remains fixed. This is equivalent to assigning an uncertainty of $100 \%$ to $r_{A}$ and taking the upper limit. The results for this new scenario are presented on the right hand side of figure 23, where the shifts $\delta \gamma_{C K M}$ have been halved with respect to those found on the left hand side of the same figure, however the absolute numerical values of about $\pm 5^{\circ}$, still represent huge effects on the CKM angle $\gamma$ itself. 

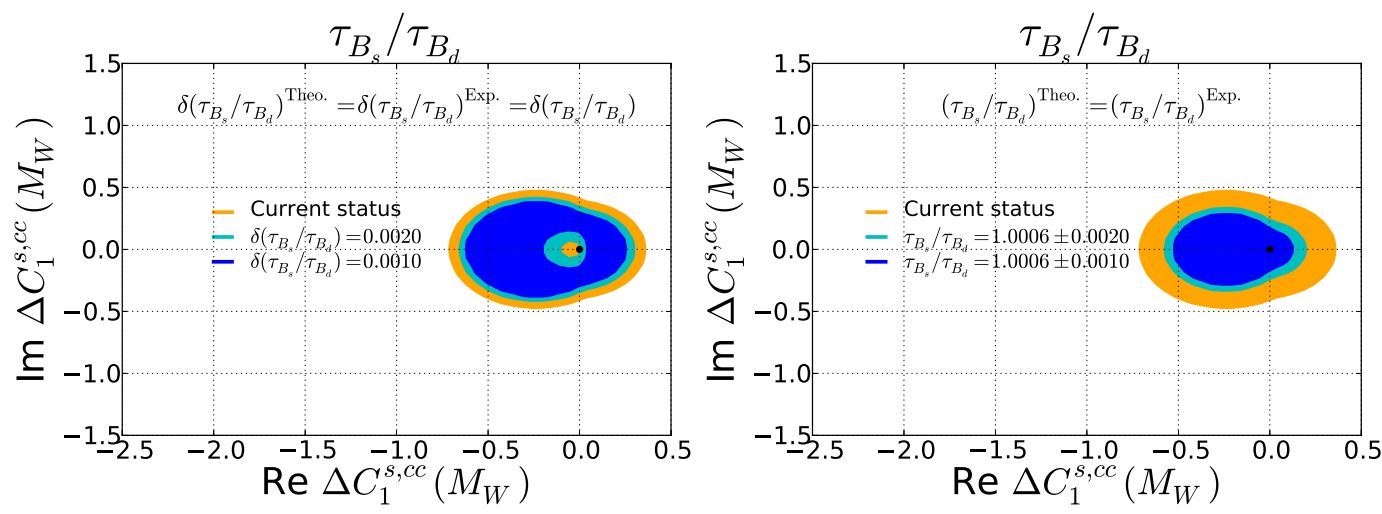

Figure 24. Future scenarios concerning the behaviour of $\tau_{B_{s}} / \tau_{B_{d}}$. In the left panel the central experimental value of the lifetime ratio is assumed to remain unchanged in the future whereas the uncertainties will be reduced. In the right panel, the theoretical and experimental values for the lifetime ratio are supposed to become equal. The black dot corresponds to the SM result.

Here clearly more theoretical work leading to a more precise understanding of $r_{A}$ and $r_{A^{\prime}}$ is highly desirable.

\section{$6 \quad$ Future prospects}

In this section we will present projections for observables, that are particularly promising to further shrink the allowed regions of NP contributions to non-leptonic tree-level decays. We have already studied the impact of BSM effects in non-leptonic tree-level decays on the observables $\Delta \Gamma_{d}$ and the CKM angle $\gamma$ in detail. More precise experimental data on $\Delta \Gamma_{d}$ will immediately lead to stronger bounds on the $\Delta B=1$ Wilson coefficients, it could also exclude the possibility of solving the D0 dimuon asymmetry with an enlarged value of $\Delta \Gamma_{d}$. Alternatively, if the measured values of $\Delta \Gamma_{d}$ will not be SM-like, we could get an intriguing hint for BSM physics. In order to make use of the extreme sensitivity of the CKM angle $\gamma$ on an imaginary part of $C_{1}$ more theory work is required to make this relation quantitatively reliable. If this is available, then already the current experimental uncertainty on $\gamma$ will exclude a large part of the allowed region on $\Delta C_{1}$ - or it will indicate the existence of NP effects. Below we will show projections for improved experimental values on the lifetime ratio $\tau_{B_{s}} / \tau_{B_{d}}$ and the semi-leptonic $\mathrm{CP}$ asymmetries, as well as commenting on consequences of our BSM approach to the recently observed flavour anomalies.

\section{$6.1 \tau_{B_{s}} / \tau_{B_{d}}$}

As already explained, the lifetime ratio $\tau_{B_{s}} / \tau_{B_{d}}$ can pose very strong constraints on the Wilson coefficients $C_{1}$ and $C_{2}$, if we e.g. assume that BSM effects are only acting in the $b \rightarrow c \bar{c} s$ channel. In figure 24 we show future projections, assuming the errors will go down to 2 per mille or even one per mille. On the l.h.s. of figure 24 we assume that the current experiment value will stay - in this case a tension between the SM value and the experimental measurement will emerge. On the r.h.s. of figure 24 we assume that the future experimental value perfectly agrees with the SM prediction. 

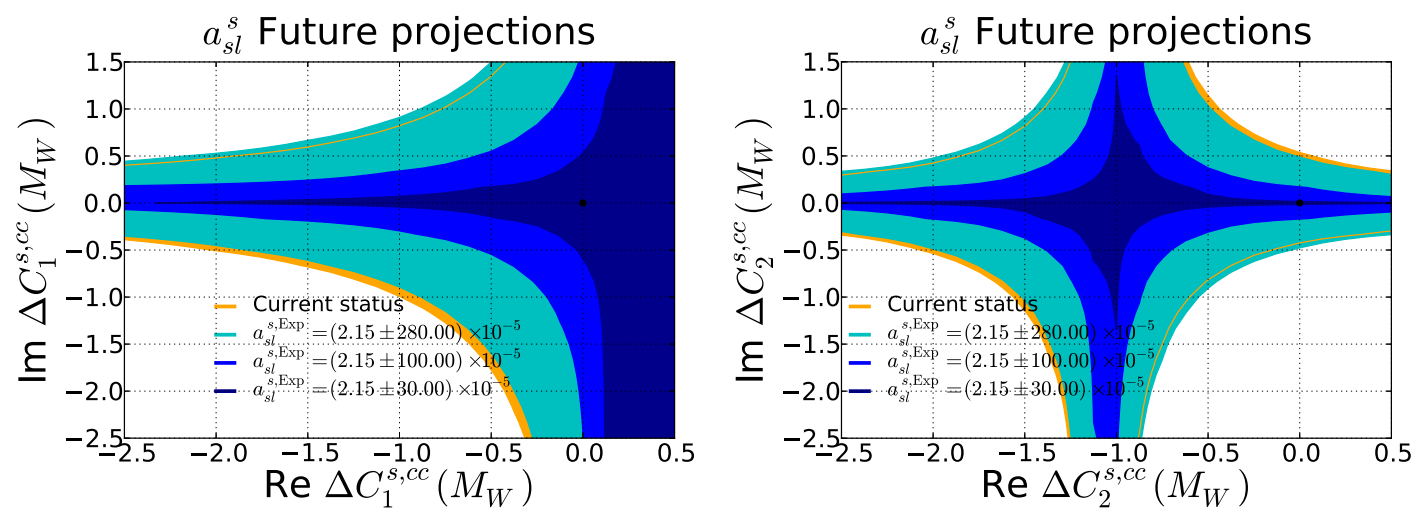

Figure 25. Future scenarios for the precision in the observable $a_{s l}^{s}$ and resulting constraints on $\Delta C_{1}$ and $\Delta C_{2}$. The current uncertainty is expected to be reduced down to 1 per mille and later even to 0.3 per mille.

\subsection{Semi-leptonic CP asymmetries}

The experimental precision for the semi-leptonic CP asymmetries is still much larger than the tiny SM values for these quantities. Nevertheless already at this stage $a_{s l}^{q}$ provide important bounds on possible BSM effects in the Wilson coefficients. The experimental precision in the semi-leptonic $\mathrm{CP}$-asymmetries will rise considerable in the near future, see e.g. table 1 of [155] from where we take:

$$
\begin{aligned}
& \delta\left(a_{s l}^{s}\right)=1 \cdot 10^{-3} \quad \text { LHCb } 2025 \\
& \delta\left(a_{s l}^{s}\right)=3 \cdot 10^{-4} \quad \text { Upgrade II }
\end{aligned}
$$

We show the dramatic impact of these future projections on the BSM bounds on the Wilson coefficients in figure 25.

\subsection{Rare decays}

As discussed in $[58,60]$ NP effects in the $b \rightarrow c \bar{c} s$ transitions can induce shifts in the Wilson coefficient of the operator

$$
\hat{Q}_{9 V}=\frac{\alpha}{4 \pi}\left(\overline{\hat{s}}_{L} \gamma_{\mu} \hat{b}_{L}\right)\left(\overline{\hat{\ell}} \gamma^{\mu} \hat{\ell}\right)
$$

leading to

$$
\left.\Delta C_{9}^{\mathrm{eff}}\right|_{\mu=m_{b}}=\left.\left[8.48 \Delta C_{1}+1.96 \Delta C_{2}\right]\right|_{\mu=M_{W}} .
$$

This result offers an interesting link with the anomalous deviations in observables associated with the decay $B \rightarrow K^{(*)} \mu^{+} \mu^{-}$, where model independent explanations with physics only in $C_{9}$ require $\left.\Delta C_{9}^{\text {eff }}\right|_{\mu=m_{b}}=-\mathcal{O}(1)$. In order to account for NP phases we use the results presented in [156] where $\Delta C_{9}$ is allowed to take complex values leading to the constraints shown in figure 26. Here both $C_{1}$ and $C_{2}$ get a shift towards negative values. BSM in effects in non-leptonic tree-level can in principle explain the deviations seen in leptonflavour universal observables, like the branching ratios or $P_{5}^{\prime}$; they can, however, not explain the anomalous values of lepton flavour universality violating observables like $R_{K}$. Future measurements will show, whether the bounds, obtained in figure 26 should be included in our full fit. 

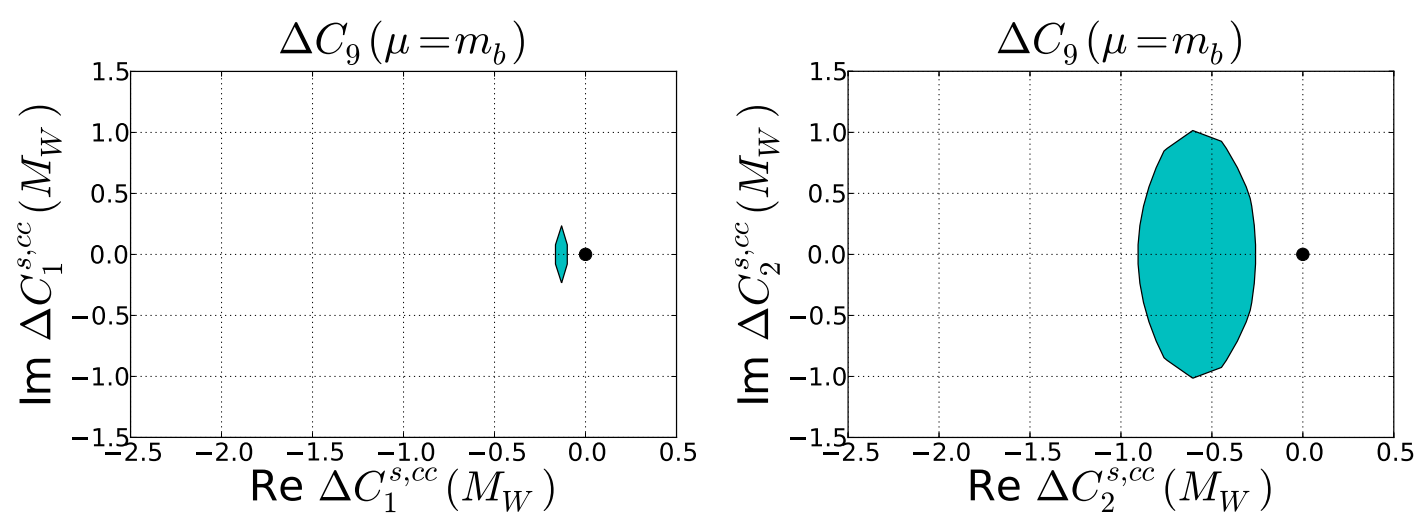

Figure 26. Regions for NP, at $90 \%$ C.L., in the $\operatorname{Re} \Delta C_{1}^{s, c c}$-Im $\Delta C_{1}^{s, c c}$ (left) and $\operatorname{Re} \Delta C_{2}^{s, c c}$ $\operatorname{Im} \Delta C_{2}^{s, c c}$ (right) planes allowed by the B physics anomalies related with the decay $B \rightarrow K^{(*)} \mu^{+} \mu^{-}$. The black dot corresponds to the SM result.

\section{Conclusions and outlook}

In this work we have questioned the well accepted assumption of having no NP in tree level decays, in particular we explored for possible deviations with respect to the SM values in the dimension six current-current operators $\hat{Q}_{1}$ (colour suppressed) and $\hat{Q}_{2}$ (colour allowed) associated with the quark level transitions $b \rightarrow q \bar{q}^{\prime} s$ and $b \rightarrow q \bar{q}^{\prime} d\left(q, q^{\prime}=u, c\right)$. We evaluated the size of the NP effects by modifying the corresponding Wilson coefficients according to $C_{1} \rightarrow C_{1}+\Delta C_{1}, C_{2} \rightarrow C_{2}+\Delta C_{2}$, for $\Delta C_{1,2} \in \mathcal{C}$; we found that sizeable deviations in $\Delta C_{1,2}$ are not ruled out by the recent experimental data.

Our analysis was based on a $\chi^{2}$-fit where we included different B-physics observables involving the decay processes: $\bar{B}_{d}^{0} \rightarrow D^{*} \pi, \bar{B}_{d}^{0} \rightarrow \pi \pi, \bar{B}_{d}^{0} \rightarrow \pi \rho, \bar{B}_{d}^{0} \rightarrow \rho \rho, \bar{B} \rightarrow X_{s} \gamma$, $\bar{B}_{s} \rightarrow J / \psi \phi$ and $\bar{B} \rightarrow X_{d} \gamma$. We also considered neutral B mixing observables: the semileptonic asymmetries $a_{s l}^{s}$ and $a_{s l}^{d}$ as well as the decay width difference $\Delta \Gamma_{s}$ of $B_{s}^{0}$ oscillations and the lifetime ratio of $B_{s}$ and $B_{d}$ mesons. Finally we also studied the CKM angles $\beta, \beta_{s}$ and $\gamma$.

For the amplitudes of the hadronic transitions $\bar{B}_{d}^{0} \rightarrow D^{*} \pi, \bar{B}_{d}^{0} \rightarrow \pi \pi, \bar{B}_{d}^{0} \rightarrow \pi \rho$ and $\bar{B}_{d}^{0} \rightarrow \rho \rho$ and $\bar{B}_{s} \rightarrow J / \psi \phi$ we used the formulas calculated within the QCD factorization framework. We have identified a high sensitivity on $\Delta C_{1,2}$ with respect to the power corrections arising in the annihilation topologies and in some cases in those for the hardspectator scattering as well. It is also important to mention that the uncertainty in the parameter $\lambda_{B}$ used to describe the inverse moment of the light cone distribution for the neutral $\mathrm{B}$ mesons is of special importance in defining the size of $\Delta C_{1}$ and $\Delta C_{2}$. For the mixing observables and the lifetime ratios we have benefited from the enormous progress achieved in the precision of the hadronic input parameters, thus we have also updated the corresponding SM predictions:

$$
\begin{aligned}
\Delta M_{s} & =(18.77 \pm 0.86) \mathrm{ps}^{-1}, & \Delta M_{d} & =(0.543 \pm 0.029) \mathrm{ps}^{-1}, \\
\Delta \Gamma_{s} & =(9.1 \pm 1.3) \cdot 10^{-2} \mathrm{ps}^{-1}, & \Delta \Gamma_{d} & =(2.6 \pm 0.4) \cdot 10^{-3} \mathrm{ps}^{-1}, \\
a_{s l}^{s} & =(2.06 \pm 0.18) \cdot 10^{-5}, & a_{s l}^{d} & =(-4.73 \pm 0.42) \cdot 10^{-4} .
\end{aligned}
$$


We have made a channel by channel study by combining different constraints for the decay chains $b \rightarrow u \bar{u} d, b \rightarrow c \bar{u} d, b \rightarrow c \bar{c} s$ and $b \rightarrow c \bar{c} d$; we also performed a universal $\chi^{2}$-fit where we have included observables mediated by $b \rightarrow q q^{\prime} s$ decays as well. The universal $\chi^{2}$-fit provides the strongest bounds on the NP deviations, we found that

$$
\begin{array}{rlrl}
\left|\operatorname{Re}\left(\Delta C_{1}\right)\right| & \leq \mathcal{O}(0.4), & & \left|\operatorname{Re}\left(\Delta C_{2}\right)\right| \leq \mathcal{O}(0.1), \\
\left|\operatorname{Im}\left(\Delta C_{1}\right)\right| \leq \mathcal{O}(0.5), & & \left|\operatorname{Im}\left(\Delta C_{2}\right)\right| \leq \mathcal{O}(0.04),
\end{array}
$$

whereas for the independent channel analyses the corresponding deviations can much larger.

We have analysed the implications of having NP in tree level b quark transitions on the decay width difference of neutral $B_{d}^{0}$ mixing $\Delta \Gamma_{d}$ - note, that the most recent experimental average is still consistent with zero. We found that enhancements in $\Delta \Gamma_{d}$ with respect to its SM value of up to a factor of five are consistent with the current experimental data. Such a huge enhancement could solve the tension between experiment and theory in the D0 measurement for the dimuon asymmetry. Thus we strongly encourage further experimental efforts to measure $\Delta \Gamma_{d}$, see also [157].

Next we evaluated the impact of our allowed NP regions for $\Delta C_{1}$ and $\Delta C_{2}$ on the determination of the CKM phase $\gamma$, where the absence of penguins leads in principle to an exceptional theoretical cleanness. We found that $\gamma$ is highly sensitive to the imaginary components of $\Delta C_{1}$ and $\Delta C_{2}$ and our BSM effects could lead to deviations in this quantity by up to $10^{\circ}$. It has to be stressed, however, that for quantitative statements about the size of the shift $\delta \gamma$ the ratios of the matrix elements $\left\langle\bar{D}^{0} K^{-}\left|Q_{1}^{\bar{u} c s}\right| B^{-}\right\rangle /\left\langle\bar{D}^{0} K^{-}\left|Q_{2}^{\bar{u} c s}\right| B^{-}\right\rangle$and $\left\langle D^{0} K^{-}\left|Q_{1}^{\bar{c} u s}\right| B^{-}\right\rangle /\left\langle D^{0} K^{-}\left|Q_{2}^{\bar{c} u s}\right| B^{-}\right\rangle$have to be determined in future with more reliable methods. So far only naive estimates are available for these ratios.

Finally we studied future projections for observables that will shrink the allowed region for NP effects - or identify a BSM region — in non-leptonic tree-level decays. Here $\tau\left(B_{s}\right) / \tau\left(B_{d}\right)$ and the semi-leptonic CP asymmetries seem to be very promising.

\section{Acknowledgments}

We would like to thank Martin Wiebusch for collaborating at early stages of the project. This work benefited from physics discussions with Christoph Bobeth, Tobias Huber, Joachim Brod, Leonardo Vernazza, Michael Spannowsky, Marco Gersabeck, Thomas Rauh, Marcel Merk, Niels Tuning, Patrick Koppenburg and Laurent Dufour. We acknowledge Luiz Vale for his help with CKM-Fitter Live. We thank to Bert Schellekens for his help and support in accessing the Nikhef computing cluster "Stoomboot" during the development of this project and to Patrick Koppenburg for supporting the access in the final stages of the project as well. GTX acknowledges support from the NWO program 156, "Higgs as Probe and Portal" and by the Deutsche Forschungsgemeinschaft (DFG, German Research Foundation) under grant 396021762 - TRR 257. The work of AL was supported by STFC via the IPPP grant. 


\begin{tabular}{|c|c|c|c|c|c|c|}
\hline Parameter & Value & Unit Ref. & Parameter & Value & Unit & Ref. \\
\hline \multicolumn{7}{|c|}{ Lepton masses, gauge boson masses and couplings } \\
\hline$m_{\mu}$ & $0.1056583745(24)$ & $\mathrm{GeV} \quad[116]$ & $G_{F}$ & $1.1663787(6) \cdot 10^{-5}$ & $\mathrm{GeV}^{-2}$ & {$[116]$} \\
\hline$m_{\tau}$ & $1.77686(12)$ & $\mathrm{GeV} \quad[116]$ & $\alpha_{s}\left(M_{Z}\right)$ & $0.1181 \pm 0.0011$ & & {$[116]$} \\
\hline$M_{Z}$ & $91.1876(21)$ & $\mathrm{GeV} \quad[116]$ & $\alpha$ & $7.2973525664(17) \cdot 10^{-3}$ & & {$[116]$} \\
\hline \multirow[t]{2}{*}{$M_{W}$} & $80.379(12)$ & $\mathrm{GeV} \quad[116]$ & $\Lambda_{5}^{\mathrm{QCD}}$ & $0.210 \pm 0.014$ & $\mathrm{GeV}$ & {$[116]$} \\
\hline & & & $\hbar$ & $6.582119514(40) \cdot 10^{-25}$ & $\mathrm{GeV} \mathrm{s}$ & {$[116]$} \\
\hline \multicolumn{7}{|l|}{ CKM } \\
\hline$\left|V_{u s}\right|$ & $0.224746_{-0.000058}^{+0.000253}$ & & $\gamma$ & $\left(65.17_{-3.05}^{+0.26}\right)^{\circ}$ & & \\
\hline$\left|V_{c b}\right|$ & $0.04243_{-0.00088}^{+0.00036}$ & & $\sin (2 \beta)_{\text {dir. }}$ & $0.699 \pm 0.017$ & & {$[111]$} \\
\hline$\left|V_{u b} / V_{c b}\right|$ & $0.08833 \pm 0.00218$ & & $\sin (2 \beta)_{\text {indir. }}$ & $0.732 \pm 0.029$ & & \\
\hline \multicolumn{7}{|c|}{ Quark masses } \\
\hline$m_{d}$ & $0.00467_{-0.00017}^{+0.00048}$ & $\mathrm{GeV} \quad[116]$ & $m_{b}^{1 S}$ & $4.65 \pm 0.03$ & $\mathrm{GeV}$ & {$[116]$} \\
\hline$m_{s}(2 \mathrm{GeV})$ & $0.093_{-0.005}^{+0.011}$ & $\mathrm{GeV} \quad[116]$ & & & & \\
\hline $\bar{m}_{c}\left(\bar{m}_{c}\right)$ & $1.27 \pm 0.02$ & $\mathrm{GeV} \quad[116]$ & & & & \\
\hline $\bar{m}_{c}\left(\bar{m}_{b}\right)$ & $0.96 \pm 0.02$ & $\mathrm{GeV}$ & $m_{t}^{\text {pole }}$ & $173.1 \pm 0.9$ & $\mathrm{GeV}$ & {$[116,158]$} \\
\hline $\bar{m}_{b}\left(\bar{m}_{b}\right)$ & $4.214_{-0.043}^{+0.042}$ & $\mathrm{GeV} \quad[159]$ & $\bar{m}_{t}\left(\bar{m}_{t}\right)$ & $163.3 \pm 0.9$ & $\mathrm{GeV}$ & \\
\hline$m_{b}^{\text {pole }}$ & $4.61 \pm 0.05$ & $\mathrm{GeV}$ & $m_{t}\left(m_{W}\right)$ & $172.6 \pm 1.0$ & $\mathrm{GeV}$ & \\
\hline
\end{tabular}

Table 17. Values of the input parameters used for our numerical evaluations.

\section{A Numerical inputs}

In this section we collect the numerical values of the input parameter used in this work.

Using the PDG value for the strong coupling

$$
\alpha_{s}\left(M_{Z}\right)=0.1181 \pm 0.0011
$$

we derive with $M_{Z}=91.1876 \pm 0.0021 \mathrm{GeV}$ at NLO-QCD

$$
\Lambda_{\mathrm{QCD}}^{(5)}=228 \pm 14 \mathrm{MeV} \text {, }
$$

while PDG gives

$$
\Lambda_{\mathrm{QCD}}^{(5)}=210 \pm 14 \mathrm{MeV},
$$

using 4-loop running, 3-loop matching. We decided to use the latter value, the effects on $\alpha_{s}\left(m_{b}\right)$ are very small.

For quark masses we use the PDG values in the MSbar definition, except for the b-quark, where we use a more conservative determination. The PDG value reads for comparison

$$
m_{b}\left(m_{b}\right)=4.18_{-0.03}^{+0.04} \mathrm{GeV}
$$

The PDG value for $m_{c}\left(m_{c}\right)$ correspond to $m_{c}\left(m_{b}\right)=0.947514$, which will be used ${ }^{9}$ for the analysis of the mixing quantities $\Delta \Gamma_{q}$ and $a_{s l}^{q}$.

\footnotetext{
${ }^{9}$ Actually $\bar{z}:=m_{c}^{2}\left(m_{b}\right) / m_{b}^{2}\left(m_{b}\right)=0.0505571$ is used.
} 


\begin{tabular}{|c|c|c|c|c|c|c|c|}
\hline Parameter & Value & Unit & Ref. & Parameter & Value & Unit & Ref. \\
\hline \multicolumn{8}{|c|}{$B$ - and light meson properties (cont.) } \\
\hline$m_{B^{+}}$ & $5279.33(13)$ & $\mathrm{MeV}$ & {$[116]$} & $a_{1}^{\pi}$ & 0.0 & & {$[160,161]$} \\
\hline$m_{B_{d}}$ & $5279.64(13)$ & $\mathrm{MeV}$ & [116] & $a_{2}^{\pi}$ & $0.17 \pm 0.10$ & & [161] \\
\hline$m_{B_{s}}$ & $5366.88(17)$ & $\mathrm{MeV}$ & [116] & $a_{1}^{\rho}$ & 0.0 & & [162] \\
\hline$m_{\pi^{+}}$ & $139.57061(24)$ & $\mathrm{MeV}$ & [116] & $a_{1 \perp}^{\rho}$ & 0.0 & & [162] \\
\hline$m_{\pi^{0}}$ & $134.9770(5)$ & $\mathrm{MeV}$ & [116] & $a_{2}^{\rho}$ & $0.1 \pm 0.3$ & & {$[20]$} \\
\hline$m_{\rho^{+}}$ & $775.11 \pm 0.34$ & $\mathrm{MeV}$ & {$[116]$} & $a_{2 \perp}^{\rho}$ & $0.11 \pm 0.05$ & & {$[162,163]$} \\
\hline$m_{\omega}$ & $782.65 \pm 0.12$ & $\mathrm{MeV}$ & [116] & $a_{1 \|}^{\phi}$ & 0 & & [164] \\
\hline$m_{D^{*+}}$ & $2010.26 \pm 0.05$ & $\mathrm{MeV}$ & [116] & $a_{2 \|}^{\phi}$ & $0 \pm 0.1$ & & [164] \\
\hline$m_{K^{0}}$ & $497.611 \pm 0.013$ & $\mathrm{MeV}$ & [116] & $B_{3}^{s} / B_{1}^{s}$ & $1.006 \pm 0.066$ & & \\
\hline$f_{B_{u, d}}$ & $190.0 \pm 1.3$ & $\mathrm{MeV}$ & [165] & $B_{R_{0}}^{s} / B_{1}^{s}$ & $0.377 \pm 0.154$ & & \\
\hline$f_{B_{d}}^{2} B_{1}^{d}$ & $(0.0305 \pm 0.0011)$ & $\mathrm{GeV}^{2}$ & & $B_{R_{1}}^{s} / B_{1}^{s}$ & $1.193 \pm 0.052$ & & \\
\hline$f_{B_{s}}^{2} B_{1}^{s}$ & $(0.0452 \pm 0.0014)$ & $\mathrm{GeV}^{2}$ & & $B_{R_{2}}^{s} / B_{1}^{s}$ & $0.318 \pm 0.118$ & & \\
\hline$f_{\pi}$ & $130.2 \pm 0.8$ & $\mathrm{MeV}$ & [165] & $B_{R_{3}}^{s} / B_{1}^{s}$ & $0.389 \pm 0.130$ & & \\
\hline$f_{\rho}$ & $216 \pm 3$ & $\mathrm{MeV}$ & {$[107,166]$} & $B_{\tilde{R}_{1}}^{s} / B_{1}^{s}$ & $1.130 \pm 0.047$ & & \\
\hline$f_{\rho}^{\perp}(1 \mathrm{GeV})$ & $165 \pm 9$ & $\mathrm{MeV}$ & {$[162,166]$} & & & & \\
\hline$f_{\omega}$ & $195 \pm 3$ & $\mathrm{MeV}$ & {$[167]$} & $B_{3}^{d} / B_{1}^{d}$ & $0.928 \pm 0.072$ & & \\
\hline$f_{D^{*}}$ & $223.5 \pm 8.4$ & $\mathrm{MeV}$ & [168] & $B_{R_{0}}^{d} / B_{1}^{d}$ & $0.383 \pm 0.156$ & & \\
\hline$F_{+}^{B \rightarrow \pi}(0)$ & $0.261 \pm 0.023$ & & {$[117,169]$} & $B_{R_{1}}^{d} / B_{1}^{d}$ & $1.190 \pm 0.060$ & & \\
\hline$A_{0}^{B \rightarrow \rho}(0)$ & $0.36 \pm 0.04$ & & {$[170]$} & $B_{R_{2}}^{d} / B_{1}^{d}$ & $0.323 \pm 0.120$ & & \\
\hline$A_{0}^{B \rightarrow D^{*}}$ & $0.66 \pm 0.02$ & & {$[171,172]$} & $B_{R_{3}}^{d} / B_{1}^{d}$ & $0.395 \pm 0.132$ & & \\
\hline$A_{0}^{B \rightarrow \phi}\left(m_{J / \psi}^{2}\right)$ & $0.68 \pm 0.07$ & & {$[170]$} & $B_{\tilde{R}_{1}}^{d} / B_{1}^{d}$ & $1.190 \pm 0.060$ & & \\
\hline$A_{1}^{B \rightarrow \phi}\left(m_{J / \psi}^{2}\right)$ & $0.37 \pm 0.04$ & & [170] & & & & \\
\hline$A_{2}^{B \rightarrow \phi}\left(m_{J / \psi}^{2}\right)$ & $0.40 \pm 0.14$ & & [170] & $\tau\left(B_{s}^{0}\right)$ & $1.509 \pm 0.004$ & ps & [111] \\
\hline$V_{2}^{B \rightarrow \phi}\left(m_{J / \psi}^{2}\right)$ & $0.70 \pm 0.06$ & & [170] & $\tau\left(B_{d}^{0}\right)$ & $1.520 \pm 0.004$ & ps & {$[111]$} \\
\hline$\Lambda_{h}$ & 500 & $\mathrm{MeV}$ & {$[20]$} & $\Gamma_{\omega}$ & $8.49 \pm 0.08$ & $\mathrm{MeV}$ & [116] \\
\hline$\lambda_{B}$ & $400 \pm 150$ & $\mathrm{MeV}$ & [107] & $\Gamma_{\rho}$ & $149.5 \pm 1.3$ & $\mathrm{MeV}$ & [116] \\
\hline
\end{tabular}

Table 18. Values of the input parameters used for our numerical evaluations (cont.).

For the top quark pole mass we use the result obtained from cross-section measurements given in [116]

$$
m_{t}^{\text {Pole }}=173.1 \pm 0.9 \mathrm{GeV}
$$

which is an average including measurements from D0, ATLAS and CMS.

Entering eq. (A.5) in the version 3 of the software RunDec [173] we obtain

$$
\bar{m}_{t}\left(\bar{m}_{t}\right)=163.3 \pm 0.9 \mathrm{GeV},
$$

and

$$
m_{t}\left(M_{W}\right)=172.6 \pm 1.0 \mathrm{GeV}
$$

We use the averages of the $B$ mixing bag parameters obtained in [65] based on the HQET sum rule calculations in $[82,90,91,174]$ and the corresponding lattice studies 
in $[175-179]$ :

$$
\begin{array}{ll}
B_{1}^{s}\left(\mu_{b}\right)=0.849 \pm 0.023, & B_{1}^{d}\left(\mu_{b}\right)=0.835 \pm 0.028 \\
B_{2}^{s}\left(\mu_{b}\right)=0.835 \pm 0.032, & B_{2}^{d}\left(\mu_{b}\right)=0.791 \pm 0.034 \\
B_{3}^{s}\left(\mu_{b}\right)=0.854 \pm 0.051, & B_{3}^{d}\left(\mu_{b}\right)=0.775 \pm 0.054 \\
B_{4}^{s}\left(\mu_{b}\right)=1.031 \pm 0.035, & B_{4}^{d}\left(\mu_{b}\right)=1.063 \pm 0.041, \\
B_{5}^{s}\left(\mu_{b}\right)=0.959 \pm 0.031, & B_{5}^{d}\left(\mu_{b}\right)=0.994 \pm 0.037,
\end{array}
$$

at the scale $\mu_{b}=\bar{m}_{b}\left(\bar{m}_{b}\right)$. For the first time we do not have to rely on vacuum insertion approximation for the dimension seven operators, instead we can now use the values obtained in $[96,179]$

$$
\begin{aligned}
& B_{R_{0}}^{q}=0.32 \pm 0.13 \\
& B_{R_{1}}^{q}=1.031 \pm 0.035 \\
& B_{\tilde{R}_{1}}^{q}=0.959 \pm 0.031 \\
& B_{R_{2}}^{q}=0.27 \pm 0.10 \\
& B_{R_{3}}^{q}=0.33 \pm 0.11
\end{aligned}
$$

Note that our notation for the dimension seven Bag parameter $B_{R_{2}}^{q}$ and $B_{R_{3}}^{q}$ corresponds to the primed bag parameter of [96]. For the remaining two operators we are using equations of motion [83]

$$
\begin{aligned}
& B_{\tilde{R_{2}}}^{q}=-B_{R_{2}}^{q} \\
& B_{\tilde{R_{3}}}^{q}=\frac{7}{5} B_{R_{3}}^{q}-\frac{2}{5} B_{R_{2}}^{q} .
\end{aligned}
$$

For the determination of the uncertainties of the ratios of Bag parameter, we first symmetrized the errors of the individual bag parameter. Based on the updated value for the bag parameter $B_{1}^{q}$ given above and the lattice average $\left(N_{f}=2+1+1\right)$ for $f_{B_{q}}$ presented in [165] — based on [92-95]

$$
\begin{aligned}
& f_{B_{s}}=(230.3 \pm 1.3) \mathrm{MeV}, \\
& f_{B_{d}}=(190.0 \pm 1.3) \mathrm{MeV},
\end{aligned}
$$

we obtain after symmetrizing the uncertainties

$$
\begin{aligned}
& f_{B_{s}}^{2} B_{1}^{s}=(0.0452 \pm 0.0014) \mathrm{GeV}^{2} \\
& f_{B_{d}}^{2} B_{1}^{d}=(0.0305 \pm 0.0011) \mathrm{GeV}^{2}
\end{aligned}
$$

Additionally, for the determination of the contributions of the double insertion of the $\Delta B=1$ effective Hamiltonians to $M_{12}^{d}$ we require the following Bag parameters at the scale $\mu_{c}=1.5 \mathrm{GeV}$ (see [82])

$$
B_{1}^{d}(1.5 \mathrm{GeV})=0.910_{-0.031}^{+0.023}, \quad B_{2}^{d}(1.5 \mathrm{GeV})=0.923_{-0.035}^{+0.029} .
$$


To calculate the CKM-elements in eq. (A.20) we require the renormalization group invariant bag parameter $\hat{B}_{1}^{s}$ which in the $\overline{\mathrm{MS}}-\mathrm{NDR}$ scheme relates with $B_{1}^{s}$, via (see e.g. [66])

$$
\begin{aligned}
\hat{B}_{1}^{s} & =\alpha_{s}(\mu)^{-\gamma_{0} /\left(2 \beta_{0}\right)}\left[1+\frac{\alpha_{s}(\mu)}{4 \pi}\left(\frac{\beta_{1} \gamma_{0}-\beta_{0} \gamma_{1}}{2 \beta_{0}^{2}}\right)\right] B_{1}^{s} \\
& =\alpha_{s}(\mu)^{-\frac{6}{23}}\left[1+\frac{\alpha_{s}(\mu)}{4 \pi} \frac{5165}{3174}\right] B_{1}^{s}=1.52734 B_{1}^{s}
\end{aligned}
$$

where we have used

$$
\begin{aligned}
C_{F} & =\frac{N_{c}^{2}-1}{2 N_{c}}, & & \\
\beta_{0} & =\frac{11 N_{c}-2 n_{f}}{3}, & \beta_{1} & =\frac{34}{3} N_{c}^{2}-\frac{10}{3} N_{c} n_{f}-2 C_{F} n_{f}, \\
\gamma_{0} & =6 \frac{N_{c}-1}{N_{c}}, & \gamma_{1} & =\frac{N_{c}-1}{2 N_{c}}\left(-21+\frac{57}{N_{c}}-\frac{19}{3} N_{c}+\frac{4}{3} n_{f}\right) .
\end{aligned}
$$

Finally we take the lifetime bag parameter from the recent HQET sum rule evaluation in [82] - here no corresponding up to date lattice evaluation exists

$$
\begin{aligned}
B_{1}\left(\mu=m_{b}\right) & =1.028_{-0.056}^{+0.064}, & B_{2}\left(\mu=m_{b}\right) & =0.988_{-0.079}^{+0.087} \\
\epsilon_{1}\left(\mu=m_{b}\right) & =-0.107_{-0.029}^{+0.028}, & \epsilon_{2}\left(\mu=m_{b}\right) & =-0.033_{-0.021}^{+0.021}
\end{aligned}
$$

Using CKMfitter-Live [153] online, we perform a fit to the CKM elements $\left|V_{u s}\right|,\left|V_{u b}\right|,\left|V_{c b}\right|$ and the CKM angle $\gamma$ excluding in all the cases the direct determination of the CKM angle $\gamma$ itself. Our inputs coincide mostly with the CKMfitter-Summer 2018 analysis, however in order to be consistent with our main study we modify the following entries $\bar{m}_{t}\left(\bar{m}_{t}\right)$, $\bar{m}_{c}\left(\bar{m}_{c}\right), \hat{B}_{1}^{s}$ and the ratios

$$
\frac{\hat{B}_{1}^{s}}{\hat{B}_{1}^{d}}=0.987 \pm 0.008[91], \quad \frac{f_{B_{s}}}{f_{B_{d}}}=1.212 \pm 0.011
$$

Our results are

$$
\begin{aligned}
\left|V_{u s}\right| & =0.224746_{-0.000058}^{+0.000253}, & \left|V_{u b}\right| & =0.003741_{-0.000061}^{+0.000082} \\
\left|V_{c b}\right| & =0.04243_{-0.00088}^{+0.00036}, & \gamma & =\left(65.17_{-3.05}^{+0.26}\right)^{\circ},
\end{aligned}
$$

from which we obtain

$$
\frac{\left|V_{u b}\right|}{\left|V_{c b}\right|}=0.08833 \pm 0.00218
$$

The full set of CKM matrix elements is then calculated under the assumption of the unitarity of the $3 \times 3$ CKM matrix. 


\section{B QCD-factorization formulas}

\section{B.1 Generic parameters}

$$
\begin{aligned}
f_{V}^{\perp}(\mu) & =f_{V}^{\perp}\left(\mu_{0}\right)\left(\frac{\alpha_{s}(\mu)}{\alpha_{s}\left(\mu_{0}\right)}\right)^{\frac{C_{F}}{\beta_{0}}}, & r_{\chi}^{\pi}(\mu) & =\frac{2 m_{\pi}^{2}}{m_{b}(\mu) 2 m_{q}(\mu)}, \\
r_{\chi}^{\rho}(\mu) & =\frac{2 m_{\rho}}{m_{b}(\mu)} \frac{f_{\rho}^{\perp}(\mu)}{f_{\rho}}, & r_{\chi}^{D^{*}}(\mu) & =\frac{2 m_{D^{*}}}{m_{b}(\mu)} \frac{f_{D^{*}}^{\perp}(\mu)}{f_{D^{*}}}, \\
r_{\chi}^{K}(\mu) & =\frac{m_{K}^{2}}{m_{b}(\mu)\left(m_{q}(\mu)+m_{s}(\mu)\right)}, & A_{\pi \pi} & =i \frac{G_{F}}{\sqrt{2}} m_{B}^{2} F_{0}^{B \rightarrow \pi}(0) f_{\pi}, \\
A_{\pi \rho} & =-i \frac{G_{F}}{\sqrt{2}} m_{B}^{2} F_{0}^{B \rightarrow \pi}(0) f_{\rho}, & A_{\rho \pi} & =-i \frac{G_{F}}{\sqrt{2}} m_{B}^{2} A_{0}^{B \rightarrow \rho}(0) f_{\pi}, \\
A_{\rho \rho} & =i \frac{G_{F}}{\sqrt{2}} m_{B}^{2} A_{0}^{B \rightarrow \rho}(0) f_{\rho}, & B_{\pi \pi} & =i \frac{G_{F}}{\sqrt{2}} f_{B} f_{\pi} f_{\pi}, \\
B_{\pi \rho} & =B_{\rho \pi}=-i \frac{G_{F}}{\sqrt{2}} f_{B} f_{\pi} f_{\rho}, & B_{\rho \rho} & =i \frac{G_{F}}{\sqrt{2}} f_{B} f_{\rho} f_{\rho}, \\
\tilde{\alpha}_{4}^{p, \pi \pi / \pi \rho} & =\alpha_{4}^{p, \pi \pi / \pi \rho}+r_{\chi}^{\pi / \rho} \alpha_{6}^{p, \pi \pi / \pi \rho}, & \tilde{\alpha}_{4}^{p, \rho \pi} & =\alpha_{4}^{p, \rho \pi}-r_{\chi}^{\pi} \alpha_{6}^{p, \rho \pi}, \\
\tilde{\alpha}_{4, E W}^{\pi \pi / \pi \rho} & =\alpha_{10}^{p, \pi \pi / \pi \rho}+r_{\chi}^{\pi / \rho} \alpha_{8}^{p, \pi \pi / \pi \rho}, & \tilde{\alpha}_{4, E W}^{\rho \pi} & =\alpha_{10}^{p, \rho \pi}-r_{\chi}^{\pi} \alpha_{8}^{p, \rho \pi} .
\end{aligned}
$$

Following $[19,20]$ we take

$$
m_{q}(\mu)=\frac{m_{\pi}^{2}}{\left(2 m_{K}^{2}-m_{\pi}^{2}\right)} m_{s}(\mu),
$$

which leads to the condition $r_{\chi}^{\pi}(\mu)=r_{\chi}^{K}(\mu)$.

\section{B.1.1 Vertices for the $B \rightarrow \pi \pi, \rho \pi, \pi \rho, \rho \rho$ decays}

$$
\begin{aligned}
V_{1,2,4,10}^{\pi} & =12 \ln \frac{m_{b}}{\mu}-18+\left[-\frac{1}{2}-3 i \pi+\left(\frac{11}{2}-3 i \pi\right) a_{1}^{\pi}-\frac{21}{20} a_{2}^{\pi}\right], \\
V_{6,8}^{\pi} & =-6, \\
V_{1,2,3,9}^{\rho} & =V^{\rho}=12 \ln \frac{m_{b}}{\mu}-18+\left[-\frac{1}{2}-3 i \pi+\left(\frac{11}{2}-3 i \pi\right) a_{1}^{\rho}-\frac{21}{20} a_{2}^{\rho}\right], \\
V_{4}^{\rho} & = \begin{cases}V^{\rho} & \text { for } \bar{B}^{0} \rightarrow \pi^{+} \rho^{-}, \\
V^{\rho}-\frac{C_{5}}{C_{3}} r_{\chi}^{\rho} V_{\perp}^{\rho} & \text { for } B \rightarrow \rho \rho,\end{cases} \\
V_{\perp}^{\rho} & =9-6 i \pi+\left(\frac{19}{6}-i \pi\right) a_{2, \perp}^{\rho}, \\
V_{7}^{\rho} & =-12 \ln \frac{m_{b}}{\mu}+6-\left[-\frac{1}{2}-3 i \pi-\left(\frac{11}{2}-3 i \pi\right) a_{1}^{\rho}-\frac{21}{20} a_{2}^{\rho}\right], \\
V_{6,8}^{\rho} & =9-6 i \pi+\left(\frac{19}{6}-i \pi\right) a_{2, \perp}^{\rho}, \\
V_{10}^{\rho} & = \begin{cases}V^{\rho} & \text { for } \bar{B}^{0} \rightarrow \pi^{+} \rho^{-}, \\
V^{\rho}-\frac{C_{7}}{C_{9}} r_{\chi}^{\rho} V_{\perp}^{\rho} & \text { for } B \rightarrow \rho \rho .\end{cases}
\end{aligned}
$$




\section{B.1.2 Vertices for the $B \rightarrow J / \psi \phi$ decay}

$$
\begin{aligned}
& V_{J / \psi \phi}^{i}= \begin{cases}-18-12 \ln \frac{\mu}{m_{b}}+f_{I}^{h} & \text { for } i=1,3,9 \\
-6-12 \ln \frac{\mu}{m_{b}}+f_{I}^{h} & \text { for } i=5,7\end{cases} \\
& f_{I}^{h}= \begin{cases}f_{I}+g_{I} \cdot(1-\tilde{z}) \frac{A_{0}^{B K *}}{A_{3}^{B K *}} & \text { for } h=0 \\
f_{I} & \text { for } h= \pm\end{cases} \\
& f_{I}=\int_{0}^{1} d \xi \Phi_{\|}^{J / \psi}(\xi)\left\{\frac{2 \tilde{z} \xi}{1-\tilde{z}(1-\xi)}+(3-2 \xi) \frac{\ln \xi}{1-\xi}\right. \\
& +\left(-\frac{3}{1-\tilde{z} \xi}+\frac{1}{1-\tilde{z}(1-\xi)}-\frac{2 \tilde{z} \xi}{[1-\tilde{z}(1-\xi)]^{2}}\right) \times \tilde{z} \xi \ln [\tilde{z} \xi] \\
& \left.+\left(3(1-\tilde{z})+2 \tilde{z} \xi+\frac{2 \tilde{z}^{2} \xi^{2}}{1-\tilde{z}(1-\xi)}\right) \times \frac{\ln (1-\tilde{z})-i \pi}{1-\tilde{z}(1-\xi)}\right\} \\
& +\int_{0}^{1} d \xi \Phi_{\perp}^{J / \psi}(\xi)\left\{-4 r \frac{\ln \xi}{1-\xi}+\frac{4 \tilde{z} r \ln [\tilde{z} \xi]}{1-\tilde{z}(1-\xi)}\right. \\
& \left.-4 \tilde{z} r \frac{\ln (1-\tilde{z})-i \pi}{1-\tilde{z}(1-\xi)}\right\} \\
& g_{I}=\int_{0}^{1} d \xi \Phi_{\|}^{J / \Psi}(\xi)\left\{\frac{-4 \xi}{(1-\tilde{z})(1-\xi)} \ln \xi+\frac{\tilde{z} \xi}{(1-\tilde{z}(1-\xi))^{2}} \ln (1-\tilde{z})\right. \\
& +\left(\frac{1}{(1-\tilde{z} \xi)^{2}}-\frac{1}{(1-\tilde{z}(1-\xi))^{2}}+\frac{2(1+\tilde{z}-2 \tilde{z} \xi)}{(1-\tilde{z})(1-\tilde{z} \xi)^{2}}\right) \times \tilde{z} \xi \ln [\tilde{z} \xi] \\
& \left.-i \pi \frac{\tilde{z} \xi}{(1-\tilde{z}(1-\xi))^{2}}\right\}+\int_{0}^{1} d \xi \Phi_{\perp}^{J / \Psi}(\xi)\left\{\frac{4 r}{(1-\tilde{z})(1-\xi)} \ln \xi\right. \\
& \left.-\frac{4 r \tilde{z}}{(1-\tilde{z})(1-\tilde{z} \xi)} \ln [\tilde{z} \xi]\right\}
\end{aligned}
$$

for

$$
\tilde{z}=\frac{m_{J / \Psi}^{2}}{m_{B}^{2}}, \quad r=2 \cdot\left(\frac{m_{c}}{m_{J / \Psi}}\right)^{2} .
$$

\section{B.1.3 Penguin functions}

To simplify the following equations we have denoted $M=\pi, \rho$ when the corresponding expressions apply to both $\pi$ and $\rho$ mesons. In addition we have used

$$
s_{p}=\left(\frac{m_{p}}{m_{b}}\right)^{2},
$$

for $p=u, c$, although in practice we consider $s_{u}=0$. Notice that in the following equations the symbol "hat" does not denote an operator and is used to distinguish the different kind 
of functions under consideration.

$$
\begin{aligned}
& P_{1,2,3}^{p, M}=P_{1,2,3}^{M}=0, \\
& P_{4}^{p, \pi}=\frac{C_{F} \alpha_{s}}{4 \pi N_{c}}\left\{C_{2}\left[\frac{4}{3} \ln \frac{m_{b}}{\mu}+\frac{2}{3}-G_{\pi}\left(s_{p}\right)\right]+C_{3}\left[\frac{8}{3} \ln \frac{m_{b}}{\mu}+\frac{4}{3}-G_{\pi}(0)-G_{\pi}(1)\right]\right. \\
& +\left(C_{4}+C_{6}\right)\left[\frac{4 n_{f}}{3} \ln \frac{m_{b}}{\mu}-\left(n_{f}-2\right) G_{\pi}(0)-G_{\pi}\left(s_{c}\right)-G_{\pi}(1)\right] \\
& \left.-6 C_{8 g}^{\mathrm{eff}}\left(1+\alpha_{1}^{\pi}+\alpha_{2}^{\pi}\right)\right\} \text {, } \\
& P_{6}^{p, M}=\frac{C_{F} \alpha_{s}}{4 \pi N_{c}}\left\{C_{2}\left[\frac{4}{3} \ln \frac{m_{b}}{\mu}+\frac{2}{3}-\hat{G}_{M}\left(s_{p}\right)\right]+C_{3}\left[\frac{8}{3} \ln \frac{m_{b}}{\mu}+\frac{4}{3}-\hat{G}_{M}(0)-\hat{G}_{M}(1)\right]\right. \\
& \left.+\left(C_{4}+C_{6}\right)\left[\frac{4 n_{f}}{3} \ln \frac{m_{b}}{\mu}-\left(n_{f}-2\right) \hat{G}_{M}(0)-\hat{G}_{M}\left(s_{c}\right)-\hat{G}_{M}(1)\right]-2 C_{8 g}^{\mathrm{eff}}\right\}, \\
& P_{8}^{p, \pi}=\frac{\alpha}{9 \pi N_{c}}\left\{\left(N_{c} C_{1}+C_{2}\right)\left[\frac{4}{3} \ln \frac{m_{b}}{\mu}+\frac{2}{3}-\hat{G}_{\pi}\left(s_{p}\right)\right]-3 C_{7}^{\mathrm{eff}}\right\} \\
& P_{10}^{p, M}=\frac{\alpha}{9 \pi N_{c}}\left\{\left(N_{c} C_{1}+C_{2}\right)\left[\frac{4}{3} \ln \frac{m_{b}}{\mu}+\frac{2}{3}-G_{M}\left(s_{p}\right)\right]-9 C_{7}^{\mathrm{eff}}\left(1+\alpha_{1}^{M}+\alpha_{2}^{M}\right)\right\}, \\
& P_{4}^{p, \rho}= \begin{cases}P_{4}^{\prime p, \rho} & \text { for } \bar{B}^{0} \rightarrow \pi^{+} \rho^{-}, \\
P_{4}^{\prime p, \rho}-r_{\chi}^{\rho} P_{4}^{\prime \prime p, \rho} & \text { for } B \rightarrow \rho \rho,\end{cases} \\
& P_{4}^{\prime p, \rho}=\frac{C_{F} \alpha_{s}}{4 \pi N_{c}}\left\{C_{2}\left[\frac{4}{3} \ln \frac{m_{b}}{\mu}+\frac{2}{3}-G_{\rho}\left(s_{p}\right)\right]+C_{3}\left[\frac{8}{3} \ln \frac{m_{b}}{\mu}+\frac{4}{3}-G_{\rho}(0)-G_{\rho}(1)\right]\right. \\
& +\left(C_{4}+C_{6}\right)\left[\frac{4 n_{f}}{3} \ln \frac{m_{b}}{\mu}-\left(n_{f}-2\right) G_{\rho}(0)-G_{\rho}\left(s_{c}\right)-G_{\rho}(1)\right] \\
& \left.-6 C_{8 g}^{\mathrm{eff}}\left(1+\alpha_{1}^{\rho}+\alpha_{2}^{\rho}\right)\right\} \text {, } \\
& P_{4}^{\prime \prime p, \rho}=-\left[C_{2} \hat{G}_{\rho}\left(s_{p}\right)+C_{3}\left(\hat{G}_{\rho}(0)+\hat{G}_{\rho}(1)\right)\right. \\
& \left.+\left(C_{4}+C_{6}\right)\left(3 \hat{G}_{\rho}(0)+\hat{G}_{\rho}\left(s_{p}\right)+\hat{G}_{\rho}(1)\right)\right], \\
& P_{7,9}^{u, \rho}=\frac{\alpha}{9 \pi}\left\{( N _ { c } C _ { 1 } + C _ { 2 } ) \left[\frac{4}{3} \frac{m_{b}}{\mu}-\frac{10}{9}+\frac{4 \pi^{2}}{3} \sum_{r=\rho, \omega} \frac{f_{r}^{2}}{m_{\rho}^{2}-m_{r}^{2}+i m_{r} \Gamma_{r}}\right.\right. \\
& \left.\left.-\frac{2 \pi}{3} \frac{m_{\rho}^{2}}{t_{c}} i+\frac{2}{3} \ln \frac{m_{\rho}^{2}}{m_{b}^{2}}+\frac{2}{3} \frac{t_{c}-m_{\rho}^{2}}{t_{c}} \ln \frac{t_{c}-m_{\rho}^{2}}{m_{\rho}^{2}}\right]-3 C_{7, \gamma}^{\mathrm{eff}}\right\} \text {, } \\
& P_{7,9}^{c, \rho}=\frac{\alpha}{9 \pi}\left\{\left(N_{c} C_{1}+C_{2}\right)\left[\frac{4}{3} \ln \frac{m_{b}}{\mu}+\frac{2}{3}+\frac{4}{3} \ln \frac{m_{c}}{m_{b}}\right]-3 C_{\gamma}^{\mathrm{eff}}\right\}, \\
& P_{8}^{p, \rho}=-\frac{\alpha}{9 \pi N_{c}}\left(N_{c} C_{1}+C_{2}\right) \hat{G}_{\rho}\left(s_{p}\right), \\
& P_{10}^{p, \rho}=\frac{\alpha}{9 \pi N_{c}}\left(P_{10}^{\prime p, \rho}+r_{\chi}^{\rho} P_{10}^{\prime \prime p, \rho}\right), \\
& P_{10}^{\prime p, \rho}=\left(N_{c} C_{1}+C_{2}\right)\left[\frac{4}{3} \ln \frac{m_{b}}{\mu}+\frac{2}{3}-G_{\rho}\left(s_{p}\right)\right]-9 C_{7, \gamma}^{\mathrm{eff}}\left(1+\alpha_{1}^{\rho}+\alpha_{2}^{\rho}\right), \\
& P_{10}^{\prime \prime p, \rho}=\left(N_{c} C_{1}+C_{2}\right) \hat{G}_{\rho}\left(s_{p}\right) \text {. }
\end{aligned}
$$


For the calculation of $P_{7,9}^{u, \rho}$ above the symbol $t_{c}$ denotes

$$
t_{c}=4 \pi^{2}\left(f_{\rho}^{2}+f_{\omega}^{2}\right)
$$

Extra functions required for the evaluation of the penguin contributions

$$
\begin{aligned}
& G_{M}\left(s_{c}\right)=\frac{5}{3}-\frac{2}{3} \ln \left(s_{c}\right)+\frac{\alpha_{1}^{M}}{2}+\frac{\alpha_{2}^{M}}{5}+\frac{4}{3}\left(8+9 \alpha_{1}^{M}+9 \alpha_{2}^{M}\right) s_{c} \\
& +2\left(8+63 \alpha_{1}^{M}+214 \alpha_{2}^{M}\right) s_{c}^{2}-24\left(9 \alpha_{1}^{M}+80 \alpha_{2}^{M}\right) s_{c}^{3} \\
& +2880 \alpha_{2}^{M} s_{c}^{4}-\frac{2}{3} \sqrt{1-4 s_{c}}\left(2 \operatorname{arctanh} \sqrt{1-4 s_{c}}-i \pi\right)\left[1+2 s_{c}\right. \\
& \left.+6\left(4+27 \alpha_{1}^{M}+78 \alpha_{2}^{M}\right) s_{c}^{2}-36\left(9 \alpha_{1}^{M}+70 \alpha_{2}^{M}\right) s_{c}^{3}+4320 \alpha_{2}^{M} s_{c}^{4}\right] \\
& +12 s_{c}^{2}\left(2 \operatorname{arctanh} \sqrt{1-4 s_{c}}-i \pi\right)^{2}\left[1+3 \alpha_{1}^{M}+6 \alpha_{2}^{M}-\frac{4}{3}\left(1+9 \alpha_{1}^{\rho}\right.\right. \\
& \left.\left.+36 \alpha_{2}^{M}\right) s_{c}+18\left(\alpha_{1}^{M}+10 \alpha_{2}^{M}\right) s_{c}^{2}-240 \alpha_{2}^{M} s_{c}^{3}\right], \\
& G_{M}(0)=\frac{5}{3}+\frac{2 i \pi}{3}+\frac{\alpha_{1}^{M}}{2}+\frac{\alpha_{2}^{M}}{5}, \\
& G_{M}(1)=\frac{85}{3}-6 \sqrt{3} \pi+\frac{4 \pi^{2}}{9}-\left(\frac{155}{2}-36 \sqrt{3} \pi+12 \pi^{2}\right) \alpha_{1}^{M}+\left(\frac{7001}{5}\right. \\
& \left.-504 \sqrt{3} \pi+136 \pi^{2}\right) \alpha_{2}^{M} \\
& \hat{G}_{\pi}^{p}\left(s_{c}\right)=\frac{16}{9}\left(1-3 s_{c}\right)-\frac{2}{3}\left[\ln \left(s_{c}\right)+\left(1-4 s_{c}\right)^{3 / 2}\left(2 \arctan \sqrt{1-4 s_{c}}-i \pi\right)\right], \\
& \hat{G}_{\pi}^{p}(0)=\frac{16}{9}+\frac{2 \pi i}{3}, \\
& \hat{G}_{\pi}^{p}(1)=\frac{2 \pi}{\sqrt{3}}-\frac{32}{9} \\
& \hat{G}_{\rho}\left(s_{c}\right)=1+\frac{\alpha_{1, \perp}^{\rho}}{3}+\frac{\alpha_{2, \perp}^{\rho}}{6}-4 s_{c}\left(9+12 \alpha_{1, \perp}^{\rho}+14 \alpha_{2, \perp}^{\rho}\right)-6 s_{c}^{2}\left(8 \alpha_{1, \perp}^{\rho}\right. \\
& \left.+35 \alpha_{2, \perp}^{\rho}\right)+360 s_{c}^{3} \alpha_{2, \perp}^{\rho}+12 s_{c} \sqrt{1-4 s_{c}}\left(1+\left[1+4 s_{c}\right] \alpha_{1, \perp}^{\rho}\right. \\
& \left.+\left[1+15 s_{c}-30 s_{c}^{2}\right] \alpha_{2, \perp}^{\rho}\right)\left(2 \operatorname{arctanh} \sqrt{1-4 s_{c}}-i \pi\right) \\
& -12 s_{c}^{2}\left(1+\left[3-4 s_{c}\right] \alpha_{1, \perp}^{\rho}+2\left[3-10 s_{c}+15 s_{c}^{2}\right] \alpha_{2, \perp}^{\rho}\right) \\
& \times\left(2 \operatorname{arctanh} \sqrt{1-4 s_{c}}-i \pi\right)^{2} \text {, } \\
& \hat{G}_{\rho}(0)=1+\frac{1}{3} \alpha_{1, \perp}^{\rho}+\frac{1}{6} \alpha_{2, \perp}^{\rho} \\
& \hat{G}_{\rho}(1)=-35+4 \sqrt{3} \pi+\frac{4 \pi^{2}}{3}+\left(-\frac{287}{3}+20 \sqrt{3} \pi-\frac{4 \pi^{2}}{3}\right) \alpha_{1, \perp}^{\rho} \\
& +\left(\frac{565}{6}-56 \sqrt{3} \pi+\frac{64 \pi^{2}}{3}\right) \alpha_{2, \perp}^{\rho} .
\end{aligned}
$$




\section{B.1.4 Hard scattering functions for the $B \rightarrow \pi \pi, \rho \pi, \pi \rho, \rho \rho$ decays}

$$
\begin{aligned}
H_{1,2,4,10}^{\pi \pi}(\mu)= & \frac{B_{\pi \pi}}{A_{\pi \pi}} \frac{m_{B}}{\lambda_{B}}\left(9\left[1+a_{1}^{\pi}+a_{2}^{\pi}\right]^{2}+3 r_{\chi}^{\pi}(\mu)\left[1-a_{1}^{\pi}+a_{2}^{\pi}\right] X_{H}\right), \\
H_{6,8}^{\pi \pi}(\mu)= & 0, \\
H_{2,4,10}^{\pi \rho}(\mu)= & \frac{B_{\pi \rho}}{A_{\pi \rho}} \frac{m_{B}}{\lambda_{B}}\left(9\left[1+a_{1}^{\pi}+a_{2}^{\pi}\right]\left[1+a_{1}^{\rho}+a_{2}^{\rho}\right]+3 r_{\chi}^{\pi}(\mu)\left[1-a_{1}^{\rho}\right.\right. \\
& \left.\left.+a_{2}^{\rho}\right] X_{H}\right), \\
H_{6,8}^{\pi \rho}(\mu)= & 0, \\
H_{2,4,10}^{\rho \pi}= & \frac{B_{\rho \pi}}{A_{\rho \pi}} \frac{m_{B}}{\lambda_{B}}\left(9\left[1+a_{1}^{\pi}+a_{2}^{\pi}\right]\left[1+a_{1}^{\rho}+a_{2}^{\rho}\right]+3 r_{\chi}^{\rho}(\mu)\left[1-a_{1}^{\pi}\right.\right. \\
& \left.\left.+a_{2}^{\pi}\right]\left[3\left(1+a_{1, \perp}^{\rho}+a_{2, \perp}^{\rho}\right) X_{H}-\left(6+9 a_{1, \perp}^{\rho}+11 a_{2, \perp}^{\rho}\right)\right]\right), \\
H_{6,8}^{\rho \pi}(\mu)= & 0, \\
H_{1,2,4,9,10}^{\rho \rho}(\mu)= & \frac{B_{\rho \rho}}{A_{\rho \rho}}\left[\frac{m_{B_{d}}}{\lambda_{B}}\right]\left[9\left(1+a_{1}^{\rho}+a_{2}^{\rho}\right)^{2}+9 r_{\chi}^{\rho}(\mu)\left(1-a_{1}^{\rho}+a_{2}^{\rho}\right)\right. \\
& \left.\times\left(X_{H}-2\right)\right], \\
H_{7}^{\rho \rho}(\mu)= & -\frac{B_{\rho \rho}}{A_{\rho \rho}}\left[\frac{m_{B_{d}}}{\lambda_{B}}\right]\left[9\left(1+a_{1}^{\rho}+a_{2}^{\rho}\right)\left(1-a_{1}^{\rho}+a_{2}^{\rho}\right)+9 r_{\chi}^{\rho}(\mu)\right. \\
& \left.\times\left(1+a_{1}^{\rho}+a_{2}^{\rho}\right)\left(X_{H}-2\right)\right] .
\end{aligned}
$$

\section{B.1.5 Hard scattering function for the $B \rightarrow J / \psi \phi$}

For the amplitudes of the decay $B \rightarrow J / \psi \phi$, the spectator interaction functions depend on the polarization of the final states, for $h=0, \pm$ we have

$$
\begin{aligned}
H_{1,3,9}^{J / \psi \phi, 0}= & \frac{f_{B} f_{J / \psi} f_{\phi}}{\tilde{h}^{0}} \int_{0}^{1} d \xi \frac{\Phi_{1}^{B}(\xi)}{\xi} \int_{0}^{1} d \tilde{\xi} \frac{\Phi^{J / \Psi}(\tilde{\xi})}{\tilde{\xi}} \int_{0}^{1} d \bar{\eta} \frac{\Phi^{\phi}(\bar{\eta})}{\bar{\eta}} \\
H_{1,3,9}^{J / \psi \phi, \pm}= & \frac{2 f_{B} f_{J / \Psi} f_{\phi} m_{J / \Psi} m_{\phi}}{m_{B}^{2} \tilde{h}^{ \pm}(1-\tilde{z})} \int_{0}^{1} d \xi \frac{\Phi_{1}^{B}(\xi)}{\xi} \int_{0}^{1} d \tilde{\xi} \frac{\Phi^{J / \Psi}(\tilde{\xi})}{\tilde{\xi}} \\
& \int_{0}^{1} d \bar{\eta}\left[\frac{\Phi_{\perp}^{\phi, v}(\bar{\eta})}{\bar{\eta}} \pm \frac{\Phi_{\perp}^{\phi, a}(\bar{\eta})}{4 \bar{\eta}^{2}}\right] \\
H_{5,7}^{J / \psi \phi, h}= & -H_{1,3,9}^{J / \psi \phi, h} .
\end{aligned}
$$

The helicity functions in the denominators of eqs. (B.15) are

$$
\begin{aligned}
& \tilde{h}^{0}=\frac{f_{J / \psi}}{2 m_{\phi}}\left[\left(m_{B}^{2}-m_{J / \Psi}^{2}-m_{\phi}^{2}\right)\left(m_{B}+m_{\phi}\right) A_{1}^{B \rightarrow \phi}\left(m_{J / \psi}^{2}\right)-\frac{4 m_{B}^{2} p_{c}^{2}}{m_{B}+m_{\phi}} A_{2}^{B \rightarrow \phi}\left(m_{J / \psi}^{2}\right)\right], \\
& \tilde{h}^{ \pm}=m_{J / \psi} f_{J / \psi}\left[\left(m_{B}+m_{\phi}\right) A_{1}^{B \rightarrow \phi}\left(m_{J / \psi}^{2}\right) \pm \frac{2 m_{B} p_{c}}{m_{B}+m_{\phi}} V^{B \rightarrow \phi}\left(m_{J / \psi}^{2}\right)\right]
\end{aligned}
$$


with

$$
p_{c}=\frac{\sqrt{\left(m_{\phi}^{2}-m_{J / \psi}^{2}\right)^{2}+m_{B}^{2}\left(m_{B}^{2}-2\left[m_{J / \psi}+m_{\phi}^{2}\right]\right)}}{2 m_{B}} .
$$

The form factors $A_{1,2}^{B \rightarrow \phi}\left(m_{J / \psi}^{2}\right)$ and $V^{B \rightarrow \phi}\left(m_{J / \psi}^{2}\right)$ used for the evaluation of the functions $\tilde{h}^{0}$ and $\tilde{h}^{ \pm}$were calculated based on [169], the corresponding numerical values can be found in appendix A.

The twist-3 distribution amplitudes of the $\phi$ meson in eqs. (B.15) have been denoted by $\Phi_{\perp}^{\phi, a}(x)$ and $\Phi_{\perp}^{\phi, v}(x)$, they are given explicitly by

$$
\begin{aligned}
\Phi_{\perp}^{\phi, a}(x)= & 6 x(1-x)\left[1+a_{1}^{\|}[2 x-1]+\left\{\frac{1}{4} a_{2}^{\|}+\frac{5}{3} \zeta_{3}\left(1-\frac{3}{16} \omega_{3}^{A, \phi}\right.\right.\right. \\
& \left.\left.+\frac{9}{16} \omega_{3}^{V, \phi}\right)\right\}\left(5[2 x-1]^{2}-1\right)+6 \delta_{+}\{3 x(1-x) \\
& \left.+(1-x) \ln (1-x)+x \ln x\}+6 \delta_{-}\{(1-x) \ln (1-x)-x \ln x\}\right] \\
\Phi_{\perp}^{\phi, v}(x)= & \frac{3}{4}\left\{1+[2 x-1]^{2}\right\}+\frac{3}{2} a_{1}^{\|[}[2 x-1]^{3}+\left\{\frac{3}{7} a_{2}^{\|}+5 \zeta_{3}\right\}\left\{3[2 x-1]^{2}-1\right\} \\
& +\left\{\frac{9}{112} a_{2}^{\|}+\frac{15}{64} \zeta_{3}\left[3 \omega_{3}^{V}-\omega_{3}^{A}\right]\right\}\left\{3-30[2 x-1]^{2}+35[2 x-1]^{4}\right\} \\
& +\frac{3}{2} \delta_{+}\{2+\ln x+\ln [1-x]\}+\frac{3}{2} \delta_{-}\{2[2 x-1]+\ln (1-x)-\ln x\} .
\end{aligned}
$$

For the rest of the LCD amplitudes of the vector mesons $J / \psi$ and $\phi$ in eqs. (B.6), (B.7) and (B.15) we use the leading term in the Gegenbauer expansion

$$
\Phi^{V}(\xi)=6 \xi(1-\xi)
$$

For different hadronic parameters required for the numerical evaluation of eq. (B.18) we use [164]

$$
\zeta_{3}=0.023, \quad \omega_{3}^{A}=0, \quad \omega_{3}^{V}=3.7, \quad \delta_{+}=0.41, \quad \delta_{-}=0 .
$$

The divergences encountered when integrating the twist-3 distribution amplitudes in eqs. (B.15) are parameterized following the model in eq. (2.23). 


\section{B.2 Annihilation coefficients}

$$
\begin{aligned}
\beta_{i}^{p, M_{1} M_{2}} & =\frac{B_{M_{1} M_{2}}}{A_{M_{1} M_{2}}} b_{i}^{p, M_{1} M_{2}} \\
b_{1}^{M_{1} M_{2}} & =\frac{C_{F}}{N_{c}^{2}} C_{1} A_{1}^{i, M_{1} M_{2}} \\
b_{2}^{M_{1} M_{2}} & =\frac{C_{F}}{N_{c}^{2}} C_{2} A_{1}^{i, M_{1} M_{2}} \\
b_{3}^{p, M_{1} M_{2}} & =\frac{C_{F}}{N_{c}^{2}}\left[C_{3} A_{1}^{i, M_{1} M_{2}}+C_{5}\left(A_{3}^{i, M_{1} M_{2}}+A_{3}^{f, M_{1} M_{2}}\right)+N_{c} C_{6} A_{3}^{f, M_{1} M_{2}}\right] \\
b_{4}^{p, M_{1} M_{2}} & =\frac{C_{F}}{N_{c}^{2}}\left[C_{4} A_{1}^{i, M_{1} M_{2}}+C_{6} A_{2}^{i, M_{1} M_{2}}\right] \\
b_{3, E W}^{p, M_{1} M_{2}} & =\frac{C_{F}}{N_{c}^{2}}\left[C_{9} A_{1}^{i, M_{1} M_{2}}+C_{7}\left(A_{3}^{i, M_{1} M_{2}}+A_{3}^{f, M_{1} M_{2}}\right)+N_{c} C_{8} A_{3}^{f, M_{1} M_{2}}\right] \\
b_{4, E W}^{p, M_{1} M_{2}} & =\frac{C_{F}}{N_{c}^{2}}\left[C_{10} A_{1}^{i, M_{1} M_{2}}+C_{8} A_{2}^{i, M_{1} M_{2}}\right]
\end{aligned}
$$

\section{B.3 Annihilation kernels}

$$
\begin{aligned}
& A_{1}^{i, \pi \pi} \approx A_{2}^{i, \pi \pi} \approx 2 \pi \alpha_{s}\left(\mu_{h}\right)\left[9\left(X_{A}-4+\frac{\pi^{2}}{3}\right)+r_{\chi}^{\pi} r_{\chi}^{\pi} X_{A}^{2}\right] \\
& A_{1}^{i, \pi \rho}=A_{1}^{i, \rho \pi} \approx 6 \pi \alpha_{s}\left[3\left(X_{A}-4+\frac{\pi^{2}}{3}\right)+r_{\chi}^{\rho} r_{\chi}^{\pi}\left(X_{A}^{2}-X_{A}\right)\right] \\
& A_{2}^{i, \pi \rho}=A_{2}^{i, \rho \pi} \approx-A_{1}^{i, \pi \rho} \\
& A_{3}^{i, \pi \pi} \approx 0 \\
& A_{3}^{i, \pi \rho}=A_{3}^{i, \rho \pi} \approx 6 \pi \alpha_{s}\left[-3 r_{\chi}^{\rho}\left(X_{A}^{2}-2 X_{A}-\frac{\pi^{2}}{3}+4\right)+r_{\chi}^{\pi}\left(X_{A}^{2}-2 X_{A}+\frac{\pi^{2}}{3}\right)\right] \\
& A_{1}^{f, \pi \rho}=A_{2}^{f, \pi \rho}=A_{1}^{f, \rho \pi}=A_{2}^{f, \rho \pi}=0 \\
& A_{3}^{f, \pi \pi} \approx 12 \pi \alpha_{s} r_{\chi}^{\pi}\left(2 X_{A}^{2}-X_{A}\right) \\
& A_{3}^{f, \pi \rho} \approx-6 \pi \alpha_{s}\left[3 r_{\chi}^{\pi}\left(2 X_{A}-1\right)\left(X_{A}-2\right)+r_{\chi}^{\rho}\left(2 X_{A}^{2}-X_{A}\right)\right] \\
& A_{3}^{f, \rho \pi}=-A_{3}^{f, \pi \rho} \approx 6 \pi \alpha_{s}\left[3 r_{\chi}^{\rho}\left(2 X_{A}-1\right)\left(2-X_{A}\right)-r_{\chi}^{\pi}\left(2 X_{A}^{2}-X_{A}\right)\right] \\
& A_{1}^{i, \rho \rho}=A_{2}^{i, \rho \rho} \approx 18 \pi \alpha_{s}\left[\left(X_{A}-4+\frac{\pi^{2}}{3}\right)+\left(r_{\chi}^{\rho}\right)^{2}\left(X_{A}-2\right)^{2}\right] \\
& A_{3}^{i, \rho \rho}=0 \\
& A_{3}^{f, \rho \rho} \approx-36 \pi \alpha_{s} r_{\chi}^{\rho}\left(2 X_{A}^{2}-5 X_{A}+2\right)
\end{aligned}
$$

Open Access. This article is distributed under the terms of the Creative Commons Attribution License (CC-BY 4.0), which permits any use, distribution and reproduction in any medium, provided the original author(s) and source are credited. 


\section{References}

[1] N. Cabibbo, Unitary Symmetry and Leptonic Decays, Phys. Rev. Lett. 10 (1963) 531 [INSPIRE].

[2] M. Kobayashi and T. Maskawa, CP Violation in the Renormalizable Theory of Weak Interaction, Prog. Theor. Phys. 49 (1973) 652 [INSPIRE].

[3] J.H. Christenson, J.W. Cronin, V.L. Fitch and R. Turlay, Evidence for the $2 \pi$ Decay of the $K_{2}^{0}$ Meson, Phys. Rev. Lett. 13 (1964) 138 [InSPIRE].

[4] BABAR collaboration, Measurement of the CP-violating asymmetry amplitude sin $2 \beta$, Phys. Rev. Lett. 89 (2002) 201802 [hep-ex/0207042] [INSPIRE].

[5] Belle collaboration, An Improved measurement of mixing induced CP-violation in the neutral B meson system, Phys. Rev. D 66 (2002) 071102 [hep-ex/0208025] [INSPIRE].

[6] LHCb collaboration, Observation of CP-violation in Charm Decays, Phys. Rev. Lett. 122 (2019) 211803 [arXiv:1903.08726] [INSPIRE].

[7] M. Chala, A. Lenz, A.V. Rusov and J. Scholtz, $\Delta A_{C P}$ within the Standard Model and beyond, JHEP 07 (2019) 161 [arXiv: 1903.10490] [INSPIRE].

[8] A. Dery and Y. Nir, Implications of the LHCb discovery of CP-violation in charm decays, JHEP 12 (2019) 104 [arXiv:1909.11242] [INSPIRE].

[9] A.D. Sakharov, Violation of CP Invariance, C asymmetry, and baryon asymmetry of the universe, Sov. Phys. Usp. 34 (1991) 392 [InSPIRE].

[10] BaBAR and Belle collaborations, The Physics of the B Factories, Eur. Phys. J. C 74 (2014) 3026 [arXiv:1406.6311] [INSPIRE].

[11] K. Anikeev et al., B physics at the Tevatron: Run II and beyond, in Workshop on B Physics at the Tevatron: Run II and Beyond, (2001) [hep-ph/0201071] [INSPIRE].

[12] G. Borissov, B-physics results from Tevatron, Int. J. Mod. Phys. A 28 (2013) 1330007 [arXiv: 1304.2173] [INSPIRE].

[13] LHCb collaboration, Implications of LHCb measurements and future prospects, Eur. Phys. J. C 73 (2013) 2373 [arXiv:1208.3355] [INSPIRE].

[14] P. Koppenburg and V. Vagnoni, Precision physics with heavy-flavoured hadrons, Adv. Ser. Direct. High Energy Phys. 23 (2015) 31 [arXiv:1510.04466] [INSPIRE].

[15] ATLAS collaboration, Measurement of the CP-violating phase $\phi_{s}$ and the $B_{s}^{0}$ meson decay width difference with $B_{s}^{0} \rightarrow J / \psi \phi$ decays in ATLAS, JHEP 08 (2016) 147 [arXiv: 1601.03297] [INSPIRE].

[16] CMS collaboration, Measurement of the CP-violating weak phase $\phi_{s}$ and the decay width difference $\Delta \Gamma_{s}$ using the $B_{s}^{0} \rightarrow J / \psi \phi(1020)$ decay channel in pp collisions at $\sqrt{s}=8 \mathrm{TeV}$, Phys. Lett. B 757 (2016) 97 [arXiv: 1507.07527] [InSPIRE].

[17] M. Beneke, G. Buchalla, M. Neubert and C.T. Sachrajda, QCD factorization for $B \rightarrow \pi \pi$ decays: Strong phases and CP-violation in the heavy quark limit, Phys. Rev. Lett. 83 (1999) 1914 [hep-ph/9905312] [INSPIRE].

[18] M. Beneke, G. Buchalla, M. Neubert and C.T. Sachrajda, QCD factorization for exclusive, nonleptonic B meson decays: General arguments and the case of heavy light final states, Nucl. Phys. B 591 (2000) 313 [hep-ph/0006124] [INSPIRE]. 
[19] M. Beneke, G. Buchalla, M. Neubert and C.T. Sachrajda, QCD factorization in $B \rightarrow \pi K, \pi \pi$ decays and extraction of Wolfenstein parameters, Nucl. Phys. B 606 (2001) 245 [hep-ph/0104110] [INSPIRE].

[20] M. Beneke and M. Neubert, $Q C D$ factorization for $B \rightarrow P P$ and $B \rightarrow P V$ decays, Nucl. Phys. B 675 (2003) 333 [hep-ph/0308039] [INSPIRE].

[21] V.A. Khoze and M.A. Shifman, Heavy quarks, Sov. Phys. Usp. 26 (1983) 387 [inSPIRE].

[22] M.A. Shifman and M.B. Voloshin, Preasymptotic Effects in Inclusive Weak Decays of Charmed Particles, Sov. J. Nucl. Phys. 41 (1985) 120 [InSPIRE].

[23] I.I.Y. Bigi and N.G. Uraltsev, Gluonic enhancements in non-spectator beauty decays: An Inclusive mirage though an exclusive possibility, Phys. Lett. B 280 (1992) 271 [INSPIRE].

[24] I.I.Y. Bigi, N.G. Uraltsev and A.I. Vainshtein, Nonperturbative corrections to inclusive beauty and charm decays: QCD versus phenomenological models, Phys. Lett. B 293 (1992) 430 [Erratum ibid. 297 (1992) 477] [hep-ph/9207214] [INSPIRE].

[25] B. Blok and M.A. Shifman, The Rule of discarding $1 / N_{c}$ in inclusive weak decays. 1., Nucl. Phys. B 399 (1993) 441 [hep-ph/9207236] [INSPIRE].

[26] B. Blok and M.A. Shifman, The Rule of discarding $1 / N_{c}$ in inclusive weak decays. 2., Nucl. Phys. B 399 (1993) 459 [hep-ph/9209289] [INSPIRE].

[27] J. Chay, H. Georgi and B. Grinstein, Lepton energy distributions in heavy meson decays from QCD, Phys. Lett. B 247 (1990) 399 [InSPIRE].

[28] M.E. Luke, Effects of subleading operators in the heavy quark effective theory, Phys. Lett. B 252 (1990) 447 [INSPIRE].

[29] A. Lenz, Lifetimes and heavy quark expansion, Int. J. Mod. Phys. A30 (2015) 1543005 [arXiv: 1405.3601] [INSPIRE].

[30] R. Fleischer, Theoretical prospects for B physics, PoS FPCP2015 (2015) 002 [arXiv: 1509.00601] [INSPIRE].

[31] M. Artuso, G. Borissov and A. Lenz, CP violation in the $B_{s}^{0}$ system, Rev. Mod. Phys. 88 (2016) 045002 [Addendum ibid. 91 (2019) 049901] [arXiv: 1511.09466] [INSPIRE].

[32] T. Jubb, M. Kirk, A. Lenz and G. Tetlalmatzi-Xolocotzi, On the ultimate precision of meson mixing observables, Nucl. Phys. B 915 (2017) 431 [arXiv:1603.07770] [InSPIRE].

[33] D0 collaboration, Evidence for an Anomalous Like-Sign Dimuon Charge Asymmetry, Phys. Rev. D 82 (2010) 032001 [arXiv: 1005.2757] [INSPIRE].

[34] D0 collaboration, Evidence for an Anomalous Like-Sign Dimuon Charge Asymmetry, Phys. Rev. Lett. 105 (2010) 081801 [arXiv:1007.0395] [INSPIRE].

[35] D0 collaboration, Measurement of the anomalous like-sign dimuon charge asymmetry with $9 \mathrm{fb}^{-} 1$ of pp collisions, Phys. Rev. D 84 (2011) 052007 [arXiv:1106.6308] [InSPIRE].

[36] D0 collaboration, Study of CP-violating charge asymmetries of single muons and like-sign dimuons in pp collisions, Phys. Rev. D 89 (2014) 012002 [arXiv:1310.0447] [InSPIRE].

[37] C.W. Bauer and N.D. Dunn, Comment on new physics contributions to $\Gamma_{12}^{s}$, Phys. Lett. B 696 (2011) 362 [arXiv:1006.1629] [INSPIRE].

[38] BaBAR collaboration, Evidence for an excess of $\bar{B} \rightarrow D^{(*)} \tau^{-} \bar{\nu}_{\tau}$ decays, Phys. Rev. Lett. 109 (2012) 101802 [arXiv:1205.5442] [INSPIRE]. 
[39] LHCb collaboration, Measurement of the ratio of branching fractions $\mathcal{B}\left(\bar{B}^{0} \rightarrow D^{*+} \tau^{-} \bar{\nu}_{\tau}\right) / \mathcal{B}\left(\bar{B}^{0} \rightarrow D^{*+} \mu^{-} \bar{\nu}_{\mu}\right)$, Phys. Rev. Lett. 115 (2015) 111803 [Erratum ibid. 115 (2015) 159901] [arXiv:1506.08614] [INSPIRE].

[40] BeLLE collaboration, Measurement of the branching ratio of $\bar{B} \rightarrow D^{(*)} \tau^{-} \bar{\nu}_{\tau}$ relative to $\bar{B} \rightarrow D^{(*)} \ell^{-} \bar{\nu}_{\ell}$ decays with hadronic tagging at Belle, Phys. Rev. D 92 (2015) 072014 [arXiv: 1507.03233] [INSPIRE].

[41] BeLle collaboration, Measurement of the branching ratio of $\bar{B}^{0} \rightarrow D^{*+} \tau^{-} \bar{\nu}_{\tau}$ relative to $\bar{B}^{0} \rightarrow D^{*+} \ell^{-} \bar{\nu}_{\ell}$ decays with a semileptonic tagging method, in 51st Rencontres de Moriond on EW Interactions and Unified Theories, (2016) [arXiv:1603.06711] [INSPIRE].

[42] S. Jäger and J. Martin Camalich, Reassessing the discovery potential of the $B \rightarrow K^{*} \ell^{+} \ell^{-}$ decays in the large-recoil region: SM challenges and BSM opportunities, Phys. Rev. D 93 (2016) 014028 [arXiv: 1412.3183] [INSPIRE].

[43] W. Altmannshofer and D.M. Straub, Implications of $b \rightarrow s$ measurements, in 50th Rencontres de Moriond on EW Interactions and Unified Theories, pp. 333-338 (2015) [arXiv: 1503.06199] [INSPIRE].

[44] S. Descotes-Genon, L. Hofer, J. Matias and J. Virto, Global analysis of $b \rightarrow$ sll anomalies, JHEP 06 (2016) 092 [arXiv: 1510.04239] [INSPIRE].

[45] M. Ciuchini et al., $B \rightarrow K^{*} \ell^{+} \ell^{-}$decays at large recoil in the Standard Model: a theoretical reappraisal, JHEP 06 (2016) 116 [arXiv:1512.07157] [INSPIRE].

[46] T. Hurth, F. Mahmoudi and S. Neshatpour, On the anomalies in the latest LHCb data, Nucl. Phys. B 909 (2016) 737 [arXiv: 1603.00865] [InSPIRE].

[47] M. Algueró et al., Emerging patterns of New Physics with and without Lepton Flavour Universal contributions, Eur. Phys. J. C 79 (2019) 714 [Addendum ibid. 80 (2020) 511] [arXiv: 1903.09578] [INSPIRE].

[48] M. Ciuchini et al., New Physics in $b \rightarrow s \ell^{+} \ell^{-}$confronts new data on Lepton Universality, Eur. Phys. J. C 79 (2019) 719 [arXiv:1903.09632] [INSPIRE].

[49] A. Datta, J. Kumar and D. London, The $B$ anomalies and new physics in $b \rightarrow s e^{+} e^{-}$, Phys. Lett. B $\mathbf{7 9 7}$ (2019) 134858 [arXiv:1903.10086] [INSPIRE].

[50] J. Aebischer, W. Altmannshofer, D. Guadagnoli, M. Reboud, P. Stangl and D.M. Straub, B-decay discrepancies after Moriond 2019, Eur. Phys. J. C 80 (2020) 252 [arXiv: 1903.10434] [INSPIRE].

[51] K. Kowalska, D. Kumar and E.M. Sessolo, Implications for new physics in $b \rightarrow s \mu \mu$ transitions after recent measurements by Belle and LHCb, Eur. Phys. J. C 79 (2019) 840 [arXiv: 1903.10932] [INSPIRE].

[52] A. Arbey, T. Hurth, F. Mahmoudi, D.M. Santos and S. Neshatpour, Update on the $b \rightarrow s$ anomalies, Phys. Rev. D 100 (2019) 015045 [arXiv: 1904.08399] [INSPIRE].

[53] A. Biswas, S. Nandi, I. Ray and S.K. Patra, New physics in $b \rightarrow$ sll decays with complex Wilson coefficients, arXiv:2004.14687 [INSPIRE].

[54] J. Bhom, M. Chrzaszcz, F. Mahmoudi, M.T. Prim, P. Scott and M. White, $A$ model-independent analysis of $b \rightarrow s \mu^{+} \mu^{-}$transitions with GAMBIT's FlavBit, arXiv:2006.03489 [INSPIRE]. 
[55] C. Bobeth, U. Haisch, A. Lenz, B. Pecjak and G. Tetlalmatzi-Xolocotzi, On new physics in $\Delta \Gamma_{d}, J H E P 06$ (2014) 040 [arXiv:1404.2531] [InSPIRE].

[56] C. Bobeth, M. Gorbahn and S. Vickers, Weak annihilation and new physics in charmless $B \rightarrow M M$ decays, Eur. Phys. J. C 75 (2015) 340 [arXiv:1409.3252] [InSPIRE].

[57] J. Brod, A. Lenz, G. Tetlalmatzi-Xolocotzi and M. Wiebusch, New physics effects in tree-level decays and the precision in the determination of the quark mixing angle $\gamma$, Phys. Rev. D 92 (2015) 033002 [arXiv:1412.1446] [InSPIRE].

[58] S. Jäger, M. Kirk, A. Lenz and K. Leslie, Charming new physics in rare B-decays and mixing?, Phys. Rev. D 97 (2018) 015021 [arXiv:1701.09183] [INSPIRE].

[59] J. Aebischer, C. Bobeth, A.J. Buras and D.M. Straub, Anatomy of $\varepsilon^{\prime} / \varepsilon$ beyond the standard model, Eur. Phys. J. C $\mathbf{7 9}$ (2019) 219 [arXiv:1808.00466] [inSPIRE].

[60] S. Jäger, M. Kirk, A. Lenz and K. Leslie, Charming New B-Physics, JHEP 03 (2020) 122 [arXiv: 1910.12924] [INSPIRE].

[61] A. Crivellin, C. Gross, S. Pokorski and L. Vernazza, Correlating $\epsilon^{\prime} / \epsilon$ to hadronic $B$ decays via $\mathrm{U}(2)^{3}$ flavour symmetry, Phys. Rev. D 101 (2020) 015022 [arXiv:1909.02101] [INSPIRE].

[62] L. Calibbi, A. Crivellin, F. Kirk, C.A. Manzari and L. Vernazza, $Z^{\prime}$ models with less-minimal flavour violation, Phys. Rev. D 101 (2020) 095003 [arXiv:1910.00014] [INSPIRE].

[63] M. Wiebusch, Numerical Computation of p-values with myFitter, Comput. Phys. Commun. 184 (2013) 2438 [arXiv: 1207.1446] [INSPIRE].

[64] D. King, M. Kirk, A. Lenz and T. Rauh, $\left|V_{c b}\right|$ and $\gamma$ from B-mixing - Addendum to " $B_{s}$ mixing observables and $\left|V_{t d} / V_{t s}\right|$ from sum rules", arXiv:1911.07856 [Addendum ibid. 03 (2020) 112] [INSPIRE].

[65] L. Di Luzio, M. Kirk, A. Lenz and T. Rauh, $\Delta M_{s}$ theory precision confronts flavour anomalies, JHEP 12 (2019) 009 [arXiv:1909.11087] [INSPIRE].

[66] G. Buchalla, A.J. Buras and M.E. Lautenbacher, Weak decays beyond leading logarithms, Rev. Mod. Phys. 68 (1996) 1125 [hep-ph/9512380] [INSPIRE].

[67] M. Beneke, G. Buchalla, C. Greub, A. Lenz and U. Nierste, Next-to-leading order QCD corrections to the lifetime difference of $B_{s}$ mesons, Phys. Lett. B 459 (1999) 631 [hep-ph/9808385] [INSPIRE].

[68] J. Aebischer, M. Fael, C. Greub and J. Virto, B physics Beyond the Standard Model at One Loop: Complete Renormalization Group Evolution below the Electroweak Scale, JHEP 09 (2017) 158 [arXiv: 1704.06639] [INSPIRE].

[69] A.J. Buras, Weak Hamiltonian, CP-violation and rare decays, in Les Houches Summer School in Theoretical Physics, Session 68: Probing the Standard Model of Particle Interactions, pp. 281-539 (1998) [hep-ph/9806471] [INSPIRE].

[70] A.J. Buras, P. Gambino and U.A. Haisch, Electroweak penguin contributions to nonleptonic $\Delta F=1$ decays at $N N L O$, Nucl. Phys. B $\mathbf{5 7 0}$ (2000) 117 [hep-ph/9911250] [INSPIRE].

[71] F. Krinner, A. Lenz and T. Rauh, The inclusive decay $b \rightarrow c \bar{c} s$ revisited, Nucl. Phys. B $\mathbf{8 7 6}$ (2013) 31 [arXiv: 1305. 5390] [INSPIRE]. 
[72] E. Bagan, P. Ball, V.M. Braun and P. Gosdzinsky, Charm quark mass dependence of QCD corrections to nonleptonic inclusive B decays, Nucl. Phys. B 432 (1994) 3 [hep-ph/9408306] [INSPIRE].

[73] E. Bagan, P. Ball, B. Fiol and P. Gosdzinsky, Next-to-leading order radiative corrections to the decay $b \rightarrow c c s$, Phys. Lett. B 351 (1995) 546 [hep-ph/9502338] [inSPIRE].

[74] A. Lenz, U. Nierste and G. Ostermaier, Penguin diagrams, charmless B decays and the missing charm puzzle, Phys. Rev. D 56 (1997) 7228 [hep-ph/9706501] [inSPIRE].

[75] A. Lenz, U. Nierste and G. Ostermaier, Determination of the CKM angle gamma and $\left|V_{u b} / V_{c b}\right|$ from inclusive direct $C P$ asymmetries and branching ratios in charmless $B$ decays, Phys. Rev. D 59 (1999) 034008 [hep-ph/9802202] [INSPIRE].

[76] C. Greub and P. Liniger, The Rare decay $b \rightarrow s$ gluon beyond leading logarithms, Phys. Lett. B 494 (2000) 237 [hep-ph/0008071] [INSPIRE].

[77] C. Greub and P. Liniger, Calculation of next-to-leading QCD corrections to $b \rightarrow s g$, Phys. Rev. D 63 (2001) 054025 [hep-ph/0009144] [INSPIRE].

[78] N.G. Uraltsev, On the problem of boosting nonleptonic b baryon decays, Phys. Lett. B $\mathbf{3 7 6}$ (1996) 303 [hep-ph/9602324] [INSPIRE].

[79] M. Neubert and C.T. Sachrajda, Spectator effects in inclusive decays of beauty hadrons, Nucl. Phys. B 483 (1997) 339 [hep-ph/9603202] [INSPIRE].

[80] M. Beneke, G. Buchalla, C. Greub, A. Lenz and U. Nierste, The $B^{+}-B_{d}^{0}$ Lifetime Difference Beyond Leading Logarithms, Nucl. Phys. B 639 (2002) 389 [hep-ph/0202106] [INSPIRE].

[81] E. Franco, V. Lubicz, F. Mescia and C. Tarantino, Lifetime ratios of beauty hadrons at the next-to-leading order in QCD, Nucl. Phys. B 633 (2002) 212 [hep-ph/0203089] [INSPIRE].

[82] M. Kirk, A. Lenz and T. Rauh, Dimension-six matrix elements for meson mixing and lifetimes from sum rules, JHEP 12 (2017) 068 [Erratum ibid. 06 (2020) 162] [arXiv: 1711.02100] [INSPIRE].

[83] M. Beneke, G. Buchalla and I. Dunietz, Width Difference in the $B_{s}-\bar{B}_{s}$ System, Phys. Rev. D 54 (1996) 4419 [Erratum ibid. 83 (2011) 119902] [hep-ph/9605259] [inSPIRE].

[84] A.S. Dighe, T. Hurth, C.S. Kim and T. Yoshikawa, Measurement of the lifetime difference of $B_{d}$ mesons: Possible and worthwhile?, Nucl. Phys. B 624 (2002) 377 [hep-ph/0109088] [INSPIRE].

[85] M. Ciuchini, E. Franco, V. Lubicz, F. Mescia and C. Tarantino, Lifetime differences and $C P$-violation parameters of neutral B mesons at the next-to-leading order in QCD, JHEP 08 (2003) 031 [hep-ph/0308029] [INSPIRE].

[86] M. Beneke, G. Buchalla, A. Lenz and U. Nierste, CP asymmetry in flavor specific B decays beyond leading logarithms, Phys. Lett. B 576 (2003) 173 [hep-ph/0307344] [INSPIRE].

[87] A. Lenz and U. Nierste, Theoretical update of $B_{s}-\bar{B}_{s}$ mixing, JHEP 06 (2007) 072 [hep-ph/0612167] [INSPIRE].

[88] T. Inami and C.S. Lim, Effects of Superheavy Quarks and Leptons in Low-Energy Weak Processes $K_{L} \rightarrow \mu \bar{\mu}, K^{+} \rightarrow \pi^{+} \nu \bar{\nu}$ and $K^{0} \Leftrightarrow \bar{K}^{0}$, Prog. Theor. Phys. 65 (1981) 297 [Erratum ibid. 65 (1981) 1772] [INSPIRE]. 
[89] A.J. Buras, M. Jamin and P.H. Weisz, Leading and Next-to-leading QCD Corrections to $\epsilon$ Parameter and $B^{0}-\bar{B}^{0}$ Mixing in the Presence of a Heavy Top Quark, Nucl. Phys. B 347 (1990) 491 [INSPIRE].

[90] A.G. Grozin, R. Klein, T. Mannel and A.A. Pivovarov, $B^{0}-\bar{B}^{0}$ mixing at next-to-leading order, Phys. Rev. D 94 (2016) 034024 [arXiv: 1606.06054] [INSPIRE].

[91] D. King, A. Lenz and T. Rauh, $B_{s}$ mixing observables and $\left|V_{t d} / V_{t s}\right|$ from sum rules, JHEP 05 (2019) 034 [arXiv: 1904.00940] [INSPIRE].

[92] N.H. Christ et al., B-meson decay constants from 2+1-flavor lattice QCD with domain-wall light quarks and relativistic heavy quarks, Phys. Rev. D 91 (2015) 054502 [arXiv: 1404 .4670] [INSPIRE].

[93] ETM collaboration, Mass of the $b$ quark and $B$-meson decay constants from $N_{f}=2+1+1$ twisted-mass lattice QCD, Phys. Rev. D 93 (2016) 114505 [arXiv:1603.04306] [InSPIRE].

[94] C. Hughes, C.T.H. Davies and C.J. Monahan, New methods for B meson decay constants and form factors from lattice NRQCD, Phys. Rev. D 97 (2018) 054509 [arXiv:1711.09981] [INSPIRE].

[95] A. Bazavov et al., B- and D-meson leptonic decay constants from four-flavor lattice QCD, Phys. Rev. D 98 (2018) 074512 [arXiv:1712.09262] [INSPIRE].

[96] HPQCD collaboration, Lattice QCD matrix elements for the $B_{s}^{0}-\bar{B}_{s}^{0}$ width difference beyond leading order, Phys. Rev. Lett. 124 (2020) 082001 [arXiv:1910.00970] [INSPIRE].

[97] M. Beneke, J. Rohrer and D. Yang, Branching fractions, polarisation and asymmetries of $B \rightarrow V V$ decays, Nucl. Phys. B 774 (2007) 64 [hep-ph/0612290] [InSPIRE].

[98] M. Bartsch, G. Buchalla and C. Kraus, $B \rightarrow V_{L} V_{L}$ Decays at Next-to-Leading Order in $Q C D$, arXiv:0810.0249 [INSPIRE].

[99] M. Beneke, T. Huber and X.-Q. Li, NNLO vertex corrections to non-leptonic B decays: Tree amplitudes, Nucl. Phys. B 832 (2010) 109 [arXiv:0911.3655] [inSPIRE].

[100] V.M. Braun, D. Ivanov and G.P. Korchemsky, The B meson distribution amplitude in QCD, Phys. Rev. D 69 (2004) 034014 [hep-ph/0309330] [INSPIRE].

[101] M. Beneke and J. Rohrwild, B meson distribution amplitude from $B \rightarrow \gamma \ell \nu$, Eur. Phys. J. C 71 (2011) 1818 [arXiv:1110.3228] [INSPIRE].

[102] Y.-M. Wang, Factorization and dispersion relations for radiative leptonic B decay, JHEP 09 (2016) 159 [arXiv: 1606.03080] [INSPIRE].

[103] M. Beneke, V.M. Braun, Y. Ji and Y.-B. Wei, Radiative leptonic decay $B \rightarrow \gamma \ell \nu_{\ell}$ with subleading power corrections, JHEP 07 (2018) 154 [arXiv: 1804.04962] [INSPIRE].

[104] Y.-M. Wang and Y.-L. Shen, Subleading-power corrections to the radiative leptonic $B \rightarrow \gamma \ell \nu$ decay in QCD, JHEP 05 (2018) 184 [arXiv: 1803.06667] [INSPIRE].

[105] BeLLE collaboration, Search for $B^{+} \rightarrow \ell^{+} \nu_{\ell \gamma}$ decays with hadronic tagging using the full Belle data sample, Phys. Rev. D 91 (2015) 112009 [arXiv:1504.05831] [InSPIRE].

[106] S.J. Lee and M. Neubert, Model-independent properties of the B-meson distribution amplitude, Phys. Rev. D 72 (2005) 094028 [hep-ph/0509350] [INSPIRE].

[107] G. Bell and V. Pilipp, $B^{-} \rightarrow \pi^{-} \pi^{0} / \rho^{-} \rho^{0}$ to NNLO in QCD factorization, Phys. Rev. D 80 (2009) 054024 [arXiv:0907.1016] [INSPIRE]. 
[108] L. Hofer, D. Scherer and L. Vernazza, $B_{s} \rightarrow \phi \rho^{0}$ and $B_{s} \rightarrow \phi \pi^{0}$ as a handle on isospin-violating New physics, JHEP 02 (2011) 080 [arXiv: 1011.6319] [INSPIRE].

[109] J.D. Bjorken, Topics in B Physics, Nucl. Phys. B Proc. Suppl. 11 (1989) 325 [inSPIRE].

[110] T. Huber, S. Kränkl and X.-Q. Li, Two-body non-leptonic heavy-to-heavy decays at NNLO in QCD factorization, JHEP 09 (2016) 112 [arXiv:1606.02888] [INSPIRE].

[111] HFLAV collaboration, Averages of b-hadron, c-hadron, and $\tau$-lepton properties as of summer 2016, Eur. Phys. J. C 77 (2017) 895 [arXiv: 1612.07233] [InSPIRE].

[112] G. Banelli, R. Fleischer, R. Jaarsma and G. Tetlalmatzi-Xolocotzi, Decoding (Pseudo)-Scalar Operators in Leptonic and Semileptonic B Decays, Eur. Phys. J. C 78 (2018) 911 [arXiv: 1809.09051] [InSPIRE].

[113] M. Beneke and S. Jager, Spectator scattering at NLO in non-leptonic b decays: Tree amplitudes, Nucl. Phys. B 751 (2006) 160 [hep-ph/0512351] [INSPIRE].

[114] G. Bell, NNLO vertex corrections in charmless hadronic B decays: Imaginary part, Nucl. Phys. B 795 (2008) 1 [arXiv:0705.3127] [INSPIRE].

[115] G. Bell, NNLO vertex corrections in charmless hadronic B decays: Real part, Nucl. Phys. B 822 (2009) 172 [arXiv:0902.1915] [INSPIRE].

[116] Particle Data Group collaboration, Review of Particle Physics, Phys. Rev. D 98 (2018) 030001 [INSPIRE].

[117] S. Gonzàlez-Solís and P. Masjuan, Study of $B \rightarrow \pi \ell \nu_{\ell}$ and $B^{+} \rightarrow \eta^{(\prime)} \ell^{+} \nu_{\ell}$ decays and determination of $\left|V_{u b}\right|$, Phys. Rev. D 98 (2018) 034027 [arXiv: 1805.11262] [INSPIRE].

[118] BaBar collaboration, Study of $B \rightarrow \pi \ell \nu$ and $B \rightarrow \rho \ell \nu$ Decays and Determination of $\left|V_{u b}\right|$, Phys. Rev. D 83 (2011) 032007 [arXiv: 1005.3288] [INSPIRE].

[119] BABAR collaboration, Branching fraction and form-factor shape measurements of exclusive charmless semileptonic B decays, and determination of $\left|V_{u b}\right|$, Phys. Rev. D 86 (2012) 092004 [arXiv: 1208.1253] [INSPIRE].

[120] Belle collaboration, Measurement of the decay $B^{0} \rightarrow \pi^{-} \ell^{+} \nu$ and determination of $\left|V_{u b}\right|$, Phys. Rev. D 83 (2011) 071101 [arXiv:1012.0090] [INSPIRE].

[121] Belle collaboration, Study of Exclusive $B \rightarrow X_{u} \ell \nu$ Decays and Extraction of $\left\|V_{u b}\right\|$ using Full Reconstruction Tagging at the Belle Experiment, Phys. Rev. D 88 (2013) 032005 [arXiv:1306.2781] [INSPIRE].

[122] CLEO collaboration, A Study of Exclusive Charmless Semileptonic B Decay and $\left|V_{u b}\right|$, Phys. Rev. Lett. 99 (2007) 041802 [hep-ex/0703041] [INSPIRE].

[123] M. Kaminski, M. Misiak and M. Poradzinski, Tree-level contributions to $B \rightarrow X_{s} \gamma$, Phys. Rev. D 86 (2012) 094004 [arXiv:1209.0965] [INSPIRE].

[124] M. Czakon, P. Fiedler, T. Huber, M.a. Misiak, T. Schutzmeier and M. Steinhauser, The $\left(Q_{7}, Q_{1,2}\right)$ contribution to $\bar{B} \rightarrow X_{s} \gamma$ at $\mathcal{O}\left(\alpha_{\mathrm{s}}^{2}\right)$, JHEP 04 (2015) 168 [arXiv:1503.01791] [INSPIRE].

[125] M. Misiak and M. Steinhauser, NNLO QCD corrections to the $\bar{B} \rightarrow X_{s} \gamma$ matrix elements using interpolation in $m_{c}$, Nucl. Phys. B 764 (2007) 62 [hep-ph/0609241] [INSPIRE].

[126] K.G. Chetyrkin, M. Misiak and M. Münz, Weak radiative $B$ meson decay beyond leading logarithms, Phys. Lett. B 400 (1997) 206 [Erratum ibid. 425 (1998) 414] [hep-ph/9612313] [INSPIRE]. 
[127] S.L. Glashow, J. Iliopoulos and L. Maiani, Weak Interactions with Lepton-Hadron Symmetry, Phys. Rev. D 2 (1970) 1285 [InSPIRE].

[128] A. Lenz and U. Nierste, Numerical Updates of Lifetimes and Mixing Parameters of B Mesons, in 6th International Workshop on the CKM Unitarity Triangle, (2011) [arXiv:1102.4274] [INSPIRE].

[129] H.M. Asatrian, A. Hovhannisyan, U. Nierste and A. Yeghiazaryan, Towards next-to-next-to-leading-log accuracy for the width difference in the $B_{s}-\bar{B}_{s}$ system: fermionic contributions to order $\left(m_{c} / m_{b}\right)^{0}$ and $\left(m_{c} / m_{b}\right)^{1}$, JHEP 10 (2017) 191 [arXiv: 1709.02160] [INSPIRE].

[130] H.-Y. Cheng, Y.-Y. Keum and K.-C. Yang, $B \rightarrow J / \psi K^{*}$ decays in $Q C D$ factorization, Phys. Rev. D 65 (2002) 094023 [hep-ph/0111094] [INSPIRE].

[131] A. Lenz, M.L. Piscopo and A.V. Rusov, Contribution of the Darwin operator to non-leptonic decays of heavy quarks, arXiv:2004.09527 [INSPIRE].

[132] T. Mannel, D. Moreno and A. Pivovarov, Heavy Quark Expansion for Heavy Hadron Lifetimes: Completing the $1 / \mathrm{m}_{b}^{3}$ Corrections, arXiv:2004.09485 [INSPIRE].

[133] H. Boos, T. Mannel and J. Reuter, The Gold plated mode revisited: $\sin (2 \beta)$ and $B^{0} \rightarrow J / \psi K_{S}$ in the standard model, Phys. Rev. D 70 (2004) 036006 [hep-ph/0403085] [INSPIRE].

[134] M. Misiak et al., Updated NNLO QCD predictions for the weak radiative B-meson decays, Phys. Rev. Lett. 114 (2015) 221801 [arXiv: 1503.01789] [INSPIRE].

[135] A. Crivellin and L. Mercolli, $B \rightarrow X_{d} \gamma$ and constraints on new physics, Phys. Rev. D 84 (2011) 114005 [arXiv:1106.5499] [INSPIRE].

[136] BaBar collaboration, Study of $B \rightarrow X \gamma$ Decays and Determination of $\left|V_{t d} / V_{t s}\right|$, Phys. Rev. $D 82$ (2010) 051101 [arXiv: 1005.4087] [INSPIRE].

[137] W. Wang, $b \rightarrow s \gamma$ and $b \rightarrow d \gamma$ (B factories), in 6th International Workshop on the CKM Unitarity Triangle, (2011) [arXiv:1102.1925] [INSPIRE].

[138] A.J. Lenz, Selected Topics in Heavy Flavour Physics, J. Phys. G 41 (2014) 103001 [arXiv:1404.6197] [INSPIRE].

[139] G. Borissov and B. Hoeneisen, Understanding the like-sign dimuon charge asymmetry in $p \bar{p}$ collisions, Phys. Rev. D 87 (2013) 074020 [arXiv:1303.0175] [INSPIRE].

[140] I.I.Y. Bigi and A.I. Sanda, Notes on the Observability of CP-violations in B Decays, Nucl. Phys. B 193 (1981) 85 [INSPIRE].

[141] M. Gronau and D. London, How to determine all the angles of the unitarity triangle from $B_{d}^{0} \rightarrow D K_{s}$ and $B_{s}^{0} \rightarrow D_{\varphi}$, Phys. Lett. B 253 (1991) 483 [inSPIRE].

[142] M. Gronau and D. Wyler, On determining a weak phase from CP asymmetries in charged $B$ decays, Phys. Lett. B 265 (1991) 172 [InSPIRE].

[143] D. Atwood, I. Dunietz and A. Soni, Enhanced CP-violation with $B \rightarrow K D^{0}\left(\bar{D}^{0}\right)$ modes and extraction of the CKM angle gamma, Phys. Rev. Lett. 78 (1997) 3257 [hep-ph/9612433] [INSPIRE].

[144] D. Atwood, I. Dunietz and A. Soni, Improved methods for observing CP-violation in $B^{ \pm} \rightarrow K D$ and measuring the CKM phase gamma, Phys. Rev. D 63 (2001) 036005 [hep-ph/0008090] [INSPIRE]. 
[145] A. Giri, Y. Grossman, A. Soffer and J. Zupan, Determining gamma using $B^{ \pm} \rightarrow D K^{ \pm}$with multibody D decays, Phys. Rev. D 68 (2003) 054018 [hep-ph/0303187] [INSPIRE].

[146] LHCb collaboration, Update of the LHCb combination of the CKM angle $\gamma$, Tech. Rep. LHCb-CONF-2018-002; CERN-LHCb-CONF-2018-002 (2018).

[147] HFLAV collaboration, Averages of b-hadron, c-hadron, and $\tau$-lepton properties as of 2018, arXiv: 1909.12524 [INSPIRE].

[148] LHCb collaboration, Physics case for an LHCb Upgrade II - Opportunities in flavour physics, and beyond, in the HL-LHC era, arXiv:1808.08865 [INSPIRE].

[149] M. Blanke and A.J. Buras, Universal Unitarity Triangle 2016 and the tension between $\Delta M_{s, d}$ and $\varepsilon_{K}$ in CMFV models, Eur. Phys. J. C $\mathbf{7 6}$ (2016) 197 [arXiv:1602.04020] [INSPIRE].

[150] M. Blanke and A.J. Buras, Emerging $\Delta M_{d^{-}}$anomaly from tree-level determinations of $\left|V_{c b}\right|$ and the angle $\gamma$, Eur. Phys. J. C $\mathbf{7 9}$ (2019) 159 [arXiv: 1812.06963] [INSPIRE].

[151] J. Brod and J. Zupan, The ultimate theoretical error on $\gamma$ from $B \rightarrow D K$ decays, JHEP 01 (2014) 051 [arXiv: 1308.5663] [INSPIRE].

[152] J. Brod, Electroweak effects in the extraction of the CKM angle $\gamma$ from $B \rightarrow D \pi$ decays, Phys. Lett. B 743 (2015) 56 [arXiv:1412.3173] [INSPIRE].

[153] CKMfitter Group collaboration, CP violation and the CKM matrix: Assessing the impact of the asymmetric B factories, Eur. Phys. J. C 41 (2005) 1 [hep-ph/0406184] [INSPIRE].

[154] UTfit collaboration, The Unitarity Triangle Fit in the Standard Model and Hadronic Parameters from Lattice QCD: A Reappraisal after the Measurements of Deltam s $_{s}$ and $B R\left(B \rightarrow \tau \nu_{\tau}\right)$, JHEP 10 (2006) 081 [hep-ph/0606167] [INSPIRE].

[155] A. Cerri et al., Report from Working Group 4: Opportunities in Flavour Physics at the HL-LHC and HE-LHC, CERN Yellow Rep. Monogr. 7 (2019) 867 [arXiv:1812. 07638] [INSPIRE].

[156] A.K. Alok, B. Bhattacharya, D. Kumar, J. Kumar, D. London and S.U. Sankar, New physics in $b \rightarrow s \mu^{+} \mu^{-}$: Distinguishing models through CP-violating effects, Phys. Rev. D 96 (2017) 015034 [arXiv: 1703.09247] [inSPIRE].

[157] T. Gershon, $\Delta \Gamma_{d}$ : A Forgotten Null Test of the Standard Model, J. Phys. G 38 (2011) 015007 [arXiv: 1007.5135] [INSPIRE].

[158] ATLAS, CDF, CMS and D0 collaborations, First combination of Tevatron and LHC measurements of the top-quark mass, arXiv:1403.4427 [INSPIRE].

[159] T. Rauh, Higher-order condensate corrections to $\Upsilon$ masses, leptonic decay rates and sum rules, JHEP 05 (2018) 201 [arXiv: 1803.05477] [INSPIRE].

[160] P. Ball and R. Zwicky, SU(3) breaking of leading-twist $K$ and $K^{*}$ distribution amplitudes: $A$ Reprise, Phys. Lett. B 633 (2006) 289 [hep-ph/0510338] [INSPIRE].

[161] P. Ball, V.M. Braun and A. Lenz, Higher-twist distribution amplitudes of the K meson in QCD, JHEP 05 (2006) 004 [hep-ph/0603063] [INSPIRE].

[162] P. Ball, V.M. Braun and A. Lenz, Twist-4 distribution amplitudes of the $K^{*}$ and phi mesons in $Q C D$, JHEP 08 (2007) 090 [arXiv:0707.1201] [INSPIRE]. 
[163] P. Ball and G.W. Jones, Twist-3 distribution amplitudes of $K^{*}$ and phi mesons, JHEP 03 (2007) 069 [hep-ph/0702100] [INSPIRE].

[164] P. Ball and V.M. Braun, Handbook of higher twist distribution amplitudes of vector mesons in QCD, in 3rd Workshop on Continuous Advances in QCD (QCD 98), pp. 125-141 (1998) [hep-ph/9808229] [INSPIRE].

[165] Flavour Lattice Averaging Group collaboration, FLAG Review 2019: Flavour Lattice Averaging Group (FLAG), Eur. Phys. J. C 80 (2020) 113 [arXiv: 1902.08191] [InSPIRE].

[166] P. Ball, G.W. Jones and R. Zwicky, $B \rightarrow V \gamma$ beyond QCD factorisation, Phys. Rev. D 75 (2007) 054004 [hep-ph/0612081] [INSPIRE].

[167] P. Ball and R. Zwicky, $B_{d, s} \rightarrow \rho, \omega, K^{*}, \phi$ decay form-factors from light-cone sum rules revisited, Phys. Rev. D 71 (2005) 014029 [hep-ph/0412079] [InSPIRE].

[168] ETM collaboration, Masses and decay constants of $D_{(s)}^{*}$ and $B_{(s)}^{*}$ mesons with $N_{f}=2+1+1$ twisted mass fermions, Phys. Rev. D 96 (2017) 034524 [arXiv: 1707.04529] [INSPIRE].

[169] A. Bharucha, Two-loop Corrections to the Bton Form Factor from QCD Sum Rules on the Light-Cone and $\left|V_{u b}\right|$, JHEP 05 (2012) 092 [arXiv: 1203.1359] [INSPIRE].

[170] A. Bharucha, D.M. Straub and R. Zwicky, $B \rightarrow V \ell^{+} \ell^{-}$in the Standard Model from light-cone sum rules, JHEP 08 (2016) 098 [arXiv: 1503.05534] [INSPIRE].

[171] F.U. Bernlochner, Z. Ligeti, M. Papucci and D.J. Robinson, Combined analysis of semileptonic $B$ decays to $D$ and $D^{*}: R\left(D^{(*)}\right),\left|V_{c b}\right|$, and new physics, Phys. Rev. $D 95$ (2017) 115008 [Erratum ibid. 97 (2018) 059902] [arXiv:1703.05330] [INSPIRE].

[172] Y. Sakaki, M. Tanaka, A. Tayduganov and R. Watanabe, Testing leptoquark models in $\bar{B} \rightarrow D^{(*)} \tau \bar{\nu}$, Phys. Rev. D $8 \mathbf{8}$ (2013) 094012 [arXiv: 1309.0301] [InSPIRE].

[173] F. Herren and M. Steinhauser, Version 3 of RunDec and CRunDec, Comput. Phys. Commun. 224 (2018) 333 [arXiv:1703.03751] [INSPIRE].

[174] A.G. Grozin and R.N. Lee, Three-loop HQET vertex diagrams for $B_{0}-\bar{B}_{0}$ mixing, JHEP 02 (2009) 047 [arXiv: 0812.4522] [INSPIRE].

[175] ETM collaboration, B-physics from $N_{f}=2$ tmQCD: the Standard Model and beyond, JHEP 03 (2014) 016 [arXiv:1308.1851] [INSPIRE].

[176] Y. Aoki, T. Ishikawa, T. Izubuchi, C. Lehner and A. Soni, Neutral B meson mixings and B meson decay constants with static heavy and domain-wall light quarks, Phys. Rev. D 91 (2015) 114505 [arXiv:1406.6192] [INSPIRE].

[177] Fermilab Lattice and MILC collaborations, $B_{(s)}^{0}$-mixing matrix elements from lattice QCD for the Standard Model and beyond, Phys. Rev. D 93 (2016) 113016 [arXiv: 1602.03560] [INSPIRE].

[178] RBC/UKQCD collaboration, SU(3)-breaking ratios for $D_{(s)}$ and $B_{(s)}$ mesons, arXiv: 1812.08791 [INSPIRE].

[179] R.J. Dowdall et al., Neutral B-meson mixing from full lattice QCD at the physical point, Phys. Rev. D 100 (2019) 094508 [arXiv:1907.01025] [INSPIRE]. 